\title{
An Atom Waveguide For Interferometry With A Bose-Einstein Condensate of ${ }^{87} \mathbf{R b}$
}

\author{
Jessica Mary Reeves \\ Elkhorn, Wisconsin
}

B.A., Lawrence University, 2000

M.A., University of Virginia, 2002

A Dissertation presented to the Graduate Faculty of the University of Virginia in Candidacy for the Degree of

Doctor of Philosophy

Department of Physics

University of Virginia

May, 2006

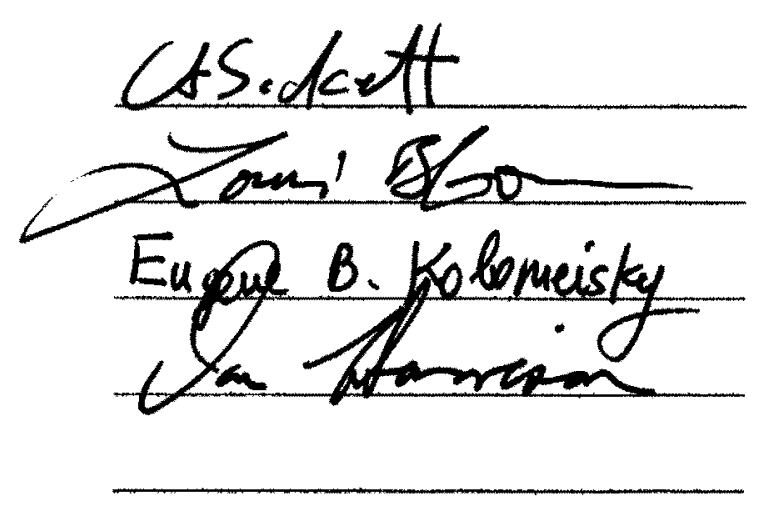


(C) Copyright by

Jessica Reeves

All Rights Reserved

May 2006 
Nature never taught me that there exists a God of glory and of infinite majesty. I had to learn that in other ways. But nature gave the word glory a meaning for me.

- C.S. Lewis 


\section{Abstract}

A Bose-Einstein condensation (BEC) production machine has been assembled and operated, using two vacuum chambers isolated from each other by a thin tube. A magnetooptical trap (MOT) is operated in the first chamber, where atoms are captured out of a thermal vapor and cooled to about $200 \mu \mathrm{K}$. The atoms are then transferred to a magnetic trap which is mounted on to a movable stage. A programmable motor moves the stage about half a meter, carrying the atoms to the second vacuum chamber where they are evaporatively cooled in a time-orbiting potential trap. We successfully used this apparatus to observe the first BEC's at the University of Virginia.

We have also implemented a novel atom trap for BEC's of ${ }^{87} \mathrm{Rb}$ to be used in atom interferometry experiments. The trap is based on a time-orbiting potential waveguide. It supports the atoms against gravity while providing weak confinement to minimize interaction effects. We have loaded a condensate into the waveguide, and removed all other confinement fields. Up to $2 \times 10^{4}$ condensate atoms have been loaded into the trap, at estimated temperatures as low as $850 \mathrm{pK}$. We expect this novel type of trap will be useful for a variety of applications in condensate interferometry.

Finally, we have characterized our trap by perturbing the atomic cloud with a sudden change in the confinement field. We subsequently obtain harmonic oscillation frequencies 
$\left(\omega_{x}, \omega_{y}, \omega_{z}\right)$ as low as $2 \pi \times(6.0,1.2,3.3) \mathrm{Hz}$. We have developed a mathematical description of the waveguide fields to account for the residual fields from the trap leads, obtaining good agreement between the measured and predicted trap behavior.

The weak confinement of our guide should greatly reduce the limiting effects of atomic interactions. We anticipate that interferometer measurement times of $1 \mathrm{~s}$ or more should be achievable in this device. With suitable modifications, our waveguide could be used to precisely measure electric polarizability, gravitational forces, rotations, and other phenomena. We expect that the trap design presented here will play an essential role in allowing condensate interferometry to realize its potential. 


\section{Acknowledgments}

Many people have inspired and supported me through this process. It would be impossible to thank them all by name, but their contributions are realized and appreciated.

I cannot begin to thank my advisor, Cass Sackett, for the opportunity to work with him. Cass is eternally patient, good-natured, and seems to asymptotically approach omniscience. It has been a privilege to be his student, and I can only hope that I am lucky enough to work with other people of his caliber in the future.

My lab mates have made the last few years unforgettable. Thank you Ken, Ofir, Ben, and Jeramy for so many memories. Also, thank you to my lunch time posse, for years of conversations both irrelevant and eminently entertaining.

The support staff in the physics department are truly a pleasure to work with. Thank you Tammie, Suzie, Dawn, and Chris for everything you do.

I also want to thank my undergraduate physics professors, David Cook and John Brandenberger, for helping me find my place. Together you encouraged and cultivated me, and I wouldn't be writing these acknowledgements today if not for you. Thank you especially to John Brandenberger for being exactly the kind of advisor that I needed, and for his continued friendship. 
My friends and family played a crucial role in my graduate school experience, by reminding me of all the other things that really matter. Thank you Sarah, Jubilee, Susan, and Ken for friendships that have meant so much to me, and that I hope will endure always. You each have a way of illuminating something profound.

Thank you to my mom, who is always there when I need someone to turn to. Thank you to my dad, who has always believed in me. You have given me the gift of confidence. And thank you to my brother Matt, who always knows what I am thinking. We are in so many ways opposite sides of the same coin. To the rest of my family, I love you all and couldn't have done any of this without you. Finally, thanks be to God, who guides me and sustains me. 


\section{Contents}

1 Introduction $\quad 1$

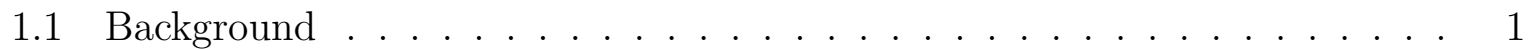

1.2 Laser Cooling of Atoms . . . . . . . . . . . . . . . . . . . . . . . . . . . . . . . . . .

1.3 Focus of Dissertation . . . . . . . . . . . . . . . . 8

2 Atom Trapping and Cooling $\quad 11$

2.1 Apparatus . . . . . . . . . . . . . . . . . . . 11

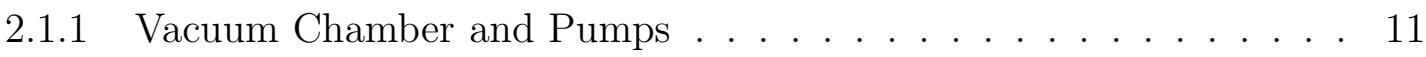

2.1.2 Lasers . . . . . . . . . . . . . . . . . . . 15

2.2 Laser Frequency Stabilization . . . . . . . . . . . . . . . . . . . . 22

2.2.1 Saturated Absorption Lock . . . . . . . . . . . . . . . . . . 23

2.2.2 Dichroic Atomic Vapor Laser Lock . . . . . . . . . . . . . . . . . . 30

2.3 Laser Cooling and the Magneto-Optical Trap . . . . . . . . . . . . . . . . 43

2.4 Magnetic Trap . . . . . . . . . . . . . . . . . . 52

3 Bose-Einstein Condensation $\quad 59$

3.1 Construction of Trap For Evaporative Cooling . . . . . . . . . . . . . . . . 59

3.2 Evaporative Cooling Principles . . . . . . . . . . . . . . . . 69

3.3 Implementation . . . . . . . . . . . . . . . . . . . . . . 73

3.3.1 TOP Trap . . . . . . . . . . . . . . . . . . . . 74

3.3.2 Successful Application of Evaporative Cooling . . . . . . . . . . . . 77

3.4 Bose-Einstein Condensation . . . . . . . . . . . . . . . . . 81

3.5 Imaging . . . . . . . . . . . . . . . . . . . . . . 88

3.5.1 Fluorescence . . . . . . . . . . . . . . . . . . . 89

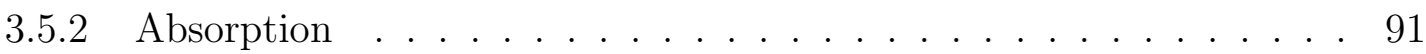

3.5.3 Calculations . . . . . . . . . . . . . . . . . . 93

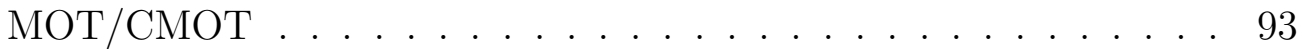

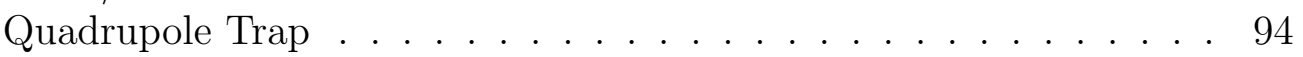

TOP Trap . . . . . . . . . . . . . . . . . . . . 99

Bose-Einstein Condensate . . . . . . . . . . . . . . 100

4 Atom Waveguide $\quad 102$

4.1 Atom Interferometry . . . . . . . . . . . . . . . . . . . . . 102

4.2 Interferometer Design . . . . . . . . . . . . . . . . . . 108 
4.2.1 Waveguide Structure and Fields . . . . . . . . . . . . . . . 111

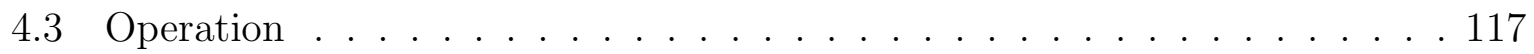

4.4 Interferometer Measurement . . . . . . . . . . . . . . . . . . 129

5 Conclusion $\quad 133$

$\begin{array}{ll}\text { Appendices } & 135\end{array}$

A Tables of Constants 136

$\begin{array}{ll}\text { B EOM Driver } & 140\end{array}$

$\begin{array}{ll}\text { C AOM Driver } & 144\end{array}$

D Lock-In Amplifier $\quad 147$

E Coil Holder Assembly $\quad 150$

$\begin{array}{lll}\text { F } & \text { Pulse Sequences } & 153\end{array}$

$\begin{array}{ll}\text { Bibliography } & 158\end{array}$ 


\section{List of Figures}

2.1 Sketch of vacuum chamber . . . . . . . . . . . . . . . . . . 12

2.2 Diagram of an acousto-optic modulator. . . . . . . . . . . . . . . . 17

2.3 Wave vector diagrams for AOM . . . . . . . . . . . . . . . . 18

2.4 Sketch of electro-optic modulator. . . . . . . . . . . . . . . . . . . 21

2.5 Laser beam path in saturated absorption spectroscopy. . . . . . . . . . . . 25

2.6 Saturated absorption in two-level system. . . . . . . . . . . . . . . 26

2.7 Saturated absorption in multi-level system. . . . . . . . . . . . . . . . 27

2.8 Laser beam path into experiment. . . . . . . . . . . . . . . . . . . . 29

2.9 DAVLL system optical path. . . . . . . . . . . . . . . . . . 32

2.10 DAVLL signal. . . . . . . . . . . . . . . . . . . . . . . . . . . . . . . . . . . 37

2.11 Temperature dependence of DAVLL signal. . . . . . . . . . . . . . . 39

2.12 Temperature sensitivity of DAVLL lock point. . . . . . . . . . . . . . . . 40

2.13 Location of temperature-independent points (TIPs) . . . . . . . . . . . . 41

2.14 Field lines for magnetic trap. . . . . . . . . . . . . . . . . . . . . . . . . . . . . . . . . . . . . . 44

2.15 Transitions driven in MOT. . . . . . . . . . . . . . . . . . . . . . 46

2.16 Fluorescence images of MOT, CMOT and magnetic trap. . . . . . . . . . 57

3.1 Sketch of magnetic trap coilholder system. . . . . . . . . . . . . . . . 61

3.2 Magnetic coils and support tower mounted to translation track. . . . . . . 67

3.3 Translation track aligned along axis of vacuum chamber. . . . . . . . . . . 67

3.4 Movement of magnetic trap to science cell. . . . . . . . . . . . . . . . . . . 68

3.5 Energies of RF field and atomic spin states in evaporative cooling. . . . . . 71

3.6 Condensate formation. . . . . . . . . . . . . . . . . . . . 87

3.7 Density plots of condensate formation. . . . . . . . . . . . . . . 87

3.8 Plots of temperature and number during evaporative cooling. . . . . . . . . 88

3.9 Fluorescence imaging system. . . . . . . . . . . . . . . . . . . . . 90

3.10 Absorption imaging system. . . . . . . . . . . . . . . . . . . . . . . 92

3.11 Gaussian fit to quadrupole distribution. . . . . . . . . . . . . . . . . . 98

4.1 Example of laser interferometer. . . . . . . . . . . . . . . . . 103

4.2 Scale drawing of trap structure. . . . . . . . . . . . . . . . . . . . . . . . . . . . . . . . . . . . . . . . . . . . .

4.3 Noise spectrum. . . . . . . . . . . . . . . . . . . . . . . . . 114

4.4 Illustration of rotating zero. . . . . . . . . . . . . . . . . . 115

4.5 Current flow through waveguide. . . . . . . . . . . . 117 
4.6 Outline of assembled waveguide structure. . . . . . . . . . . . . . . 118

4.7 Field from segment of lead. . . . . . . . . . . . . . . . . . . . . 120

4.8 Loading of Bose-Einstein condensate into the waveguide. . . . . . . . . . . 123

4.9 Measurement of $x$ oscillations. . . . . . . . . . . . . . . . . . . 125

4.10 Measurement of $y$ oscillations. . . . . . . . . . . . . . . . . . . . . . . . . . . . . . . . . . . . . . . . . . . . .

4.11 Measurement of $z$ oscillations. . . . . . . . . . . . . . . . . . . . . 127

4.12 Comparison of measured and predicted trap frequencies. . . . . . . . . . 128

4.13 Cooling below $1 \mathrm{nK}$. . . . . . . . . . . . . . . . . . . . . . . . 129

4.14 Splitting and recombining of condensate wavepackets. . . . . . . . . . . 132

A.1 Coupling constants for ${ }^{87}$ Rb. . . . . . . . . . . . . . . . . . 137

A.2 Energy level diagram for ${ }^{87}$ Rb. . . . . . . . . . . . . . . . . 138

B.1 Circuit diagram for EOM RF driver. . . . . . . . . . . . . . . . . . . . 141

B.2 Circuit diagram for EOM frequency lock. . . . . . . . . . . . . . . . . . . 142

B.3 Circuit diagrams for EOM RF driver VCO and bias control. . . . . . . . . 143

C.1 Circuit diagrams for fixed-frequency AOM driver. . . . . . . . . . . . . 145

C.2 Circuit diagram for variable-frequency AOM driver. . . . . . . . . . . . . 146

D.1 Circuit diagrams for photodiode and integrator. . . . . . . . . . . . 148

D.2 Circuit diagram for demodulator. . . . . . . . . . . . . . . . . . . 149

D.3 Circuit diagram for modulation of magnetic field, and modulation reference

for lock-in amplifier. . . . . . . . . . . . . . . . . . . . . 149

E.1 Coilholder drawing. . . . . . . . . . . . . . . . . . . . . 151

E.2 Tower drawing. . . . . . . . . . . . . . . . . . . . . . . . . 152

E.3 Mounting plate drawing. . . . . . . . . . . . . . . . . . . . . 152 


\section{List of Tables}

3.1 Initial lifetimes and heating rates. . . . . . . . . . . . . . . . . 80

3.2 Lifetime and heating after shielding. . . . . . . . . . . . . . . . . . 81

3.3 Optimized procedure for MOT, CMOT, and magnetic trap. . . . . . . . . 82

3.4 Number, temperature and density for MOT, CMOT, magnetic trap. . . . . 83

3.5 RF application during evaporative cooling. . . . . . . . . . . . . . . 83

3.6 Evaporative cooling sequence. . . . . . . . . . . . . . . . . . 84

4.1 Trap frequencies. . . . . . . . . . . . . . . . 126

A.1 Important Constants. . . . . . . . . . . . . . . . . 136

A.2 Properties of ${ }^{87} \mathrm{Rb}$. . . . . . . . . . . . . . . . . . . 136

A.3 ${ }^{87} \mathrm{Rb} \mathrm{D}_{2}\left(5^{2} \mathrm{~S}_{1 / 2} \leftrightarrow 5^{2} \mathrm{P}_{3 / 2}\right)$ Transition Properties. . . . . . . . . . . . 139

A.4 Saturation intensities. . . . . . . . . . . . . . . . . 139

F.1 Channel assignment. . . . . . . . . . . . . . . . . . . . 153

F.2 Pulse sequence used to observe condensate. . . . . . . . . . . . . . . 154

F.3 Subroutine loadcmot. . . . . . . . . . . . . . . . . . . . . . 155

F.4 Subroutine movetrap. . . . . . . . . . . . . . . . . . . . 155

F.5 Subroutine probe. . . . . . . . . . . . . . . . . 155

F.6 Evaporation routines. . . . . . . . . . . . . . . . . . 156

F.7 Pulse sequence used to load atoms into waveguide. . . . . . . . . . . . 157

F.8 Subroutine loadcmot2. . . . . . . . . . . . . . . . . . . . . 157 


\section{Chapter 1}

\section{Introduction}

The observation of Bose-Einstein condensation (BEC) in the alkali atoms rubidium [6], sodium [21], and lithium [16] in 1995 has provided the opportunity to study a macroscopic quantum system. As such, this phenomenon has opened the door to new realms of physics. BEC has implications in areas such as quantum computing, atomic clocks, navigation systems, and encryption schemes. One area of recent interest in the atomic physics community has been the prospect of a gyroscope based on a condensate interferometer. Our work has resulted in an atom waveguide well-suited for interferometry, which we hope will eventually enable measurement of inertial effects for interaction times of up to 1 s. In this dissertation I will describe the design, construction and operation of this device.

\section{$1.1 \quad$ Background}

Predicted by Albert Einstein and Satyendra Bose in 1925 [53], the theory of Bose-Einstein condensation (BEC) was for many years far ahead of experiment. After Bose developed a theory on the statistics of photons [15], Einstein was inspired to consider another type of integer-spin system - a gas of bosonic atoms. He determined that below a critical 
temperature, the free energy of the system was minimized if a finite fraction of the particles entered the lowest energy state [24]. Though the mathematics of this phase transition were worked out soon after, it was 70 years before a proper condensate was actually observed. In principle any bosonic particle can form a condensate and early efforts were focussed on ${ }^{4} \mathrm{He}$, in part because the phenomenon of superfluidity was recognized as related to BEC. While ${ }^{4} \mathrm{He}$ did prove important to condensate studies, interactions between ${ }^{4} \mathrm{He}$ atoms are strong, limiting the condensate fraction and changing the character of the transition.

In search of a candidate particle with weak interactions and larger condensate fraction, attention turned to hydrogen. The atomic interactions within a gas of spin-polarized H are so weak that there is no molecular bound state. The sample remains in the gas phase even at zero temperatures, avoiding the liquid phase where atomic interactions had hindered work with ${ }^{4} \mathrm{He}$. While early experiments cooled the hydrogen by forcing it into contact with a cryogenically cooled surface, the density of the cloud was limited due to interactions between the hydrogen and the surface. A hydrogen BEC group developed magnetic trapping techniques to avoid surface effects, and observed BEC in 1998 [25].

This was not the first observation of BEC, however. With the development of laser cooling and trapping techniques of the mid-1980's, BEC was initially achieved with other spin-polarized alkali atoms. Although laser cooling is not a realistic option with hydrogen, the other alkali atoms require laser wavelengths that are more easily accessible. Using a combination of laser cooling and magnetic trapping, alkali atoms such as rubidium and sodium are now widely used in condensate experiments. Recently metastable noble gases have also come under investigation. 
Bose-Einstein condensation occurs when the de Broglie wavelength

$$
\lambda_{d B}=\left(\frac{2 \pi \hbar^{2}}{m k_{B} T}\right)^{1 / 2}
$$

of each atom becomes comparable to the the interparticle spacing, which is about $n^{-1 / 3}$. The atomic wavefunctions begin to overlap, making the individual atoms indistinguishable from each other. This usually occurs in alkali atoms when the density is on the order of $10^{12}-10^{15}$ atoms per $\mathrm{cm}^{3}$ and the temperature reaches below $1 \mu \mathrm{K}$. In contrast, the density of air molecules at room temperature is $10^{19} \mathrm{~cm}^{-3}$. Thus while the condensate is dense in comparison with the vapor from which the constituent atoms are trapped, Bose-Einstein condensation is usually considered a dilute gaseous phenomenon.

As Eq. (1.1) shows, $\lambda_{d B}$ is inversely related to the energy of the atom. Therefore we can increase $\lambda_{d B}$ by reducing the temperature of the system. According to statistical mechanics, the transition to BEC occurs when the atoms become so cold that a significant fraction enters the ground state of the system, i.e. the occupancy of the lowest energy level becomes macroscopic. For a harmonic oscillator potential, this critical temperature is

$$
k_{B} T_{c}=\frac{\hbar \bar{\omega} N^{1 / 3}}{[\zeta(3)]^{1 / 3}} \approx 0.94 \hbar \bar{\omega} N^{1 / 3}
$$

where $\zeta$ is the Riemann zeta function and $\bar{\omega}$ is the geometric mean of the harmonic oscillator frequencies in all three dimensions

$$
\bar{\omega}=\left(\omega_{1} \omega_{2} \omega_{3}\right)^{1 / 3} .
$$


The critical temperature can also be written in terms of the peak atomic density as

$$
k_{B} T_{c}=\frac{2 \pi}{[\zeta(3 / 2)]^{2 / 3}} \frac{\hbar^{2} n^{2 / 3}}{m} \approx 3.31 \frac{\hbar^{2} n^{2 / 3}}{m} .
$$

The critical temperature varies with density, so that BEC results at different temperatures for different experiments. It is simpler to use a quantity without this dependence. The phase space density $\rho$ is defined as the number of particles contained within a volume equal to the cube of the de Broglie wavelength [53],

$$
\rho=n \lambda_{d B}^{3}=n\left(\frac{2 \pi \hbar^{2}}{m k_{B} T}\right)^{3 / 2}
$$

If we insert Equation 1.4 into Equation 1.5, we find that BEC occurs in any experiment when

$$
\rho=\zeta(3 / 2) \approx 2.612 .
$$

In order to create a condensate, then, we will require high atomic densities and long de Broglie wavelengths (effectively requiring low temperatures). For example, if our peak density is $10^{12} \mathrm{~cm}^{-3}$, we can solve for the required wavelength for condensation in a gas of ${ }^{87} \mathrm{Rb}$ as

$$
\lambda_{d B}=\left(\frac{\rho}{n}\right)^{1 / 3}=\left(\frac{2.612}{10^{12} \mathrm{~cm}^{-3}}\right)^{1 / 3}=1.4 \mu \mathrm{m} .
$$

According to Equation 1.4 this wavelength is achieved at critical temperature

$$
T_{c} \approx \frac{3.31 \hbar^{2}\left(10^{12} \mathrm{~cm}^{-3}\right)^{2 / 3}}{m k_{B}}=19 \mathrm{nK}
$$


Also, we can find the velocity of a ${ }^{87} \mathrm{Rb}$ atom at this temperature

$$
v=\sqrt{\frac{k_{B} T}{m}}=1.4 \mathrm{~mm} / \mathrm{s}
$$

The ability to remove enough kinetic energy from an atom to achieve such slow velocities is essential to BEC production. Under standard conditions of temperature and pressure, atoms have average temperature $300 \mathrm{~K}$ and velocities of up to a few hundred $\mathrm{m} / \mathrm{s}$. Cold baths of liquid nitrogen or liquid helium can only cool to about $76 \mathrm{~K}$ and $0.3 \mathrm{~K}$, respectively. The invention of laser cooling and trapping allowed the first approaches to ultracold temperatures. While this technique is thoroughly discussed in many sources, such as [42], I will briefly explain the fundamental principles at work.

\subsection{Laser Cooling of Atoms}

Laser cooling reduces an atom's kinetic energy through its interaction with photons. The momentum exchange during the process of photon absorption and emission causes a reduction in atomic velocity along the path of the photon, thus reducing that component

of the atom's kinetic energy. $E=\frac{1}{2} k T=\frac{1}{2} m v^{2}$ in each dimension, and so by slowing the atoms in all three dimensions we can reduce their temperature.

It is worth noting that the concept of temperature is not entirely appropriate to laser cooling. The thermodynamic definition of temperature applies to a system in thermal equilibrium. This is not necessarily the case during laser cooling, since there is a flux of energy through the system as photons are scattered. Nor does photon scattering qualify 
as heat exchange with the surrounding environment. Consequently, while our system may be described as achieving a steady-state, it does not meet the rigorous requirements for thermal equilibrium, and the use of temperature is not technically permissible. Nonetheless, the use of temperature as a label for a group of atoms with average kinetic energy $E$ is convenient, and so we commonly discuss the "temperature" of an atomic sample.

Laser cooling reduces the temperature, then, of an atomic cloud by reducing the average kinetic energy of the atoms and narrowing the width of the velocity distribution. Laser cooling is possible because of the strong frequency dependence of atomic absorption of radiation. The Doppler shift translates this frequency dependence into a velocity dependence, because atoms moving along the beam path will see the frequency of the light shifted according to their velocities. Absorption of laser photons provides a velocitydependent dissipative force that can be used for cooling.

There are many different laser cooling schemes, but in all of them the mechanism for cooling relies on spontaneous emission of photons. When an atom absorbs a photon, it stores the additional energy by transitioning to an excited state. The momentum $\hbar k$ of the photon causes the atom to recoil from the light source, while the photon's angular momentum is stored in the motion of the atoms internal electrons. When the atom emits a photon, whether stimulated or spontaneous, the reverse happens. During stimulated emission, the photon is emitted into the path of the laser, and no net force is applied to the atom. For a spontaneously emitted photon, the atomic recoil is in a random direction so that the net effect is a small change to the atom's momentum along the beam path. After a number of spontaneous emission events, the velocity of the atom along the beam 
path can be significantly changed.

Of course, the momentum of a photon is much smaller than the momentum of an atom. For example, consider a laser beam with wavelength $780 \mathrm{~nm}$ and an ${ }^{87} \mathrm{Rb}$ atom moving at $15 \mathrm{~m} / \mathrm{s}$, corresponding to temperature $T \sim 2 \mathrm{~K}$. The momentum of the photon is

$$
p_{\text {photon }}=\hbar k=8.5 \times 10^{-28} \mathrm{~kg} \mathrm{~m} / \mathrm{s},
$$

whereas the momentum of the atom is

$$
p_{\text {atom }}=m v=2.17 \times 10^{-24} \mathrm{~kg} \mathrm{~m} / \mathrm{s} .
$$

We see then that the momentum of the atom exceeds that of the photon by more than a factor of $10^{3}$, and the atom is not significantly slowed until it has undergone repeated cycles of absorption and emission.

In order to decelerate an atom moving in any direction, three pairs of counter-propagating beams are applied orthogonally. The laser light is commonly detuned red to lower frequencies, so that due to the Doppler effect atoms preferentially absorb light from the beam they are moving towards. As the atoms are cooled, the excess energy is carried away by spontaneously emitted photons, whose frequency is slightly bluer than the frequency of the absorbed photons since the atomic velocity at the time of emission is smaller than at the time of absorption.

As the atoms cool down, entropy flows out of the system. The only mechanism for carrying entropy away is the spontaneous fluorescence. Since the total number of photons 
is conserved and the frequency shift during emission is small, the energy of the photons changes only slightly. In contrast, the incoming laser light is tightly organized but the spontaneously emitted photons are highly disorganized, making the entropy change large. However the entropy outflow calculated by counting the number of available states is several orders of magnitude larger than the entropy lost as calculated from the temperature change of the atomic cloud, and so while laser cooling can produce large changes in the temperature of an atomic sample, it is actually an inefficient refrigerator [42].

Probably the most important property of laser cooling for BEC experiments is its ability to change the phase space density of an atomic sample. The velocity-dependent dissipative forces are not conservative, and so phase space density can be altered significantly. Thus, laser cooling is a natural candidate for use in a BEC experiment.

\subsection{Focus of Dissertation}

My research has resulted in a novel trap based on an atom waveguide. This trap supports the atoms against gravity and is designed for use in an atom interferometer. We anticipate that it will eventually allow measurement of accumulated quantum phase over interaction times of up to $1 \mathrm{~s}$.

This dissertation is divided into three main sections. Chapter 2 describes the design and assembly of our BEC experiment, and the first steps in the process of creating a condensate. I begin with discussion of the glass chamber which will contain our experiment, and how we achieve the low internal vacuum pressure needed for the experiment to work. I then describe our laser systems and the electronics used to control the beams. One of 
our lasers is frequency stabilized via a saturated absorption lock, while the other laser uses a Dichroic Atomic Vapor Laser Lock (DAVLL). I studied the temperature dependence of the DAVLL, and the results are presented. We begin the process of making a BEC by cooling and trapping ${ }^{87} \mathrm{Rb}$ atoms from a thermal vapor, using a magneto-optical trap (MOT). I discuss the operating principles of the MOT and the compressed MOT (CMOT). Finally, I introduce the principles of magnetic trapping.

Chapter 3 details condensate formation. I first describe the construction of a tightly compressed magnetic trap suitable for evaporative cooling. Once the atoms are in the magnetic trap, we move them to a second vacuum chamber and perform RF-driven evaporative cooling. I explain how the cooling works, and the equipment we used to implement it. Our evaporative cooling did not function properly at first, and I discuss some of the problems encountered. We performed several tests to diagnose the root of these problems, and eventually achieved successful evaporation and subsequently observed Bose-Einstein condensation. The condensation process is illustrated with images of our atoms and relevant calculations. Our imaging system is presented, along with the formulas used to determine parameters of the cloud, such as number of atoms, average temperature, and collision rate.

The final section of the main body is Chapter 4, where our atom waveguide is presented. I explain why we are interested in performing interferometry with Bose-Einstein condensates, due to the opportunity for enhanced phase sensitivity. We designed the waveguide specifically to ensure that our finished product is particularly suited for interferometry applications. I calculate the fields in the trap, and develop the formulas for 
the trap frequencies. We successfully loaded condensates into the waveguide, and after perturbing the cloud we observed oscillations at the trap frequencies in three dimensions. Finally, I discuss the future of this experiment, which requires a method to coherently split and recombine the condensates and measure the differential phase. 


\section{Chapter 2}

\section{Atom Trapping and Cooling}

We begin the process of making a BEC by collecting cold atoms. As I will explain in this chapter, we require a low pressure environment where the atoms will be isolated from contaminants, a laser system to perform laser cooling and trapping, and a pair of currentcarrying coils for magnetic trapping. I will also discuss our methods of laser frequency stabilization, and present the results of my study of the temperature-dependence of one of the laser locks. Finally I will discuss how we designed and implemented our atom traps.

\subsection{Apparatus}

\subsubsection{Vacuum Chamber and Pumps}

We conduct our experiment within two vacuum chambers, composed of a combination of glass and steel. A simple sketch is shown in Figure 2.1. We do not use cryogenic methods to cool the chamber. Instead, it is maintained at room temperature and the atoms within the chambers are not isolated from thermal background radiation. This is not problematic, however, because only a vanishingly small fraction of the total incident radiation is at appropriate frequencies to be absorbed by the atoms, and so the effects 


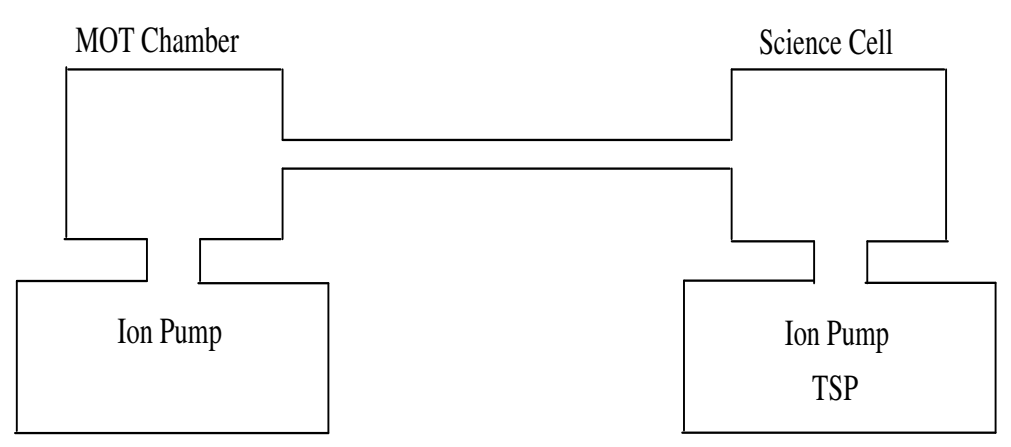

Figure 2.1: Sketch of vacuum chamber. The MOT chamber contains the Rb dispensers, and so the vapor pressure here is $10^{-9}$ torr. The science cell is separated from the MOT cell by a $30 \mathrm{~cm}$ long tube with $1 \mathrm{~cm}$ diameter, allowing a pressure gradient between the two chambers. The pressure in the science cell is $10^{-11}$ torr.

are negligible.

The first of the two chambers is designated as the MOT cell, where the atoms are trapped from a thermal vapor and undergo inital cooling. The second chamber is the science cell, where the condensates are created. Each chamber is pumped by a Star Cell ion pump from Varian, which pumps at about $20 \mathrm{~L} / \mathrm{s}$ and holds the pressure in the MOT cell around $10^{-9}$ torr. The science chamber is at an ultra-high vacuum, with pressure of about $10^{-11}$ torr. We achieve this pressure by isolating the science cell from the higherpressure MOT chamber, and attaching a $\sim 1000 \mathrm{~L} / \mathrm{s}$ Titanium sublimation pump. The details of the operation of both pumps are available from retailers, such as Kurt J. Lesker Company [1]. The two chambers are joined by a $30 \mathrm{~cm}$ long tube with $1 \mathrm{~cm}$ diameter, which isolates the chambers and allows a pressure gradient to exist between them. There is a small bellows in the middle of the tube, joining the two chambers while allowing for differential motion between them. The bellows lessens the danger of breaking the glass when the chamber is moved. 
The vacuum chambers undergo extensive preparation to make these pressures possible. As the chamber is assembled, all components, including flanges, valves and any special inserts, are scrubbed with soap and water and then wiped with methanol. Smaller components are soaked in an ultrasonic cleaner. Once clean, all components are stored in aluminum foil until needed, and whenever manipulation is necessary latex gloves are used. After assembly, the chamber is sealed and the vacuum process begins. To remove the air from the chamber, we use a set of pumps which I shall call the "bake pumping system." The first is a mechanical pump, which reduces the vacuum pressure to 500 mtorr. Attempts to run this pump any further will result in a backflow of pump oil into the system, so we then switch to a sorption pump cooled with liquid nitrogen. This brings the pressure down to $\sim 10^{-3}$ torr, at which point an ion pump replaces the sorption pump. The ion pump reduces the pressure to about $10^{-7}$ torr, but the steady release of gas molecules from the walls of the chamber prevents any further reduction of internal pressure (at least, not within a time period less than many months). The ion pump in the bake pumping system has a much higher pump rate than the ion pumps that will be used during the experiment, at $\sim 200 \mathrm{~L} / \mathrm{s}$.

In order to increase the rate of desorption from the chamber walls, we heat the chamber in a controlled bake. An oven is constructed out of ceramic "fire bricks" wrapped with aluminum foil, and the chamber is seated inside. Four steel heater rods are distributed about the chamber, without directly touching the chamber walls. We also affix four thermocouple wires to different sections of the chamber for temperature monitoring. The thermocouple and heater rod leads are extended outside the oven. The top of the oven 
is then sealed with more fire bricks, and the entire structure is draped with an insulating blanket.

Once the oven is prepared, we attach the leads of the heaters to variac current controllers and begin to heat the chamber. The current is slowly increased over several days, so that the pressure never exceeds $10^{-5}$ torr because that is the upper limit of the ion pump's operating range. We increase the current until the temperature within the oven reaches $300{ }^{\circ} \mathrm{C}$. After about a day at this elevated temperature, most of the volatile contaminant molecules have desorbed from the chamber walls and the ion pump has reduced the vapor pressure to about $10^{-6}$ torr. At this point we close the chamber's entrance valve, sealing it from the bake pumping system. We then turn off the current to the heater rods, and allow the oven to cool for about a day. The rate of heating and cooling is important, because the glass is substantially stressed during the baking process and under extreme temperature changes it can warp or shatter. As the chamber cools, the less volatile gas molecules settle and once again stick to the walls, with the vapor pressure approaching $10^{-9}$ torr.

Once the chamber is cooled, we open the oven and remove the bake pumping system. The chamber is placed on the optics bench, and the controllers for the permanent $20 \mathrm{~L} / \mathrm{s}$ ion pumps are attached. We also connect the controller for our Ti-sublimation pump (TSP), which is in the science cell. We run the TSP at $45 \mathrm{~A}$ for $\sim 5$ minutes, repeating at $50 \%$ duty cycle for one day. We continue to run the TSP once a day for about a week, and afterwards as necessary to keep the science cell pressure at $10^{-11}$ torr.

The final step in preparation of the chamber is to test our Rb source. We use two $R b$ 
dispensers, or getters, from SAES Getters USA. An aluminum mount holds two pairs of getters inside the chamber. While we only use one pair of getters at any given time, the presence of the second pair provides a safeguard against malfunction or exhaustion of the first pair. The getters are isolated from the mount itself with ceramic screws, but they are connected by wires to an electric feedthrough. When a current is run through the getters, a spray of $\mathrm{Rb}$ vapor results. However, immediately after the bake most of that spray is composed of other materials, as dirt accumulates on the getters due to handling during installation and exposure to contaminants during the bake. We find that running a current of $3 \mathrm{~A}$ through the getters for 2-3 days is sufficient to clean them. One must be careful not to run so much current that all of the rubidium is driven off, or that the vapor pressure rises above $10^{-5}$ torr, where the ion pumps may shut down. Once the chamber is prepared, installation of optics and laser alignment may begin.

\subsubsection{Lasers}

Our BEC experiment requires a high-power, easily tunable light source at $780 \mathrm{~nm}$. We also need the capability to rapidly switch the beams on and off, with switching time in the vicinity of $1 \mu \mathrm{s}$. Finally, we must have several different laser frequencies available.

To meet these needs, we use a Ti-Sapphire MBR-110 laser from Coherent, Inc. as our main light source. It provides approximately $1.2 \mathrm{~W}$ of $780 \mathrm{~nm}$ light, with a tuning range of roughly $100 \mathrm{~nm}$. As explained in Section 2.3, we need two laser frequencies in order to cool and capture atoms. We call these the "trapping" and "repump" frequencies. Instead of using two separate lasers, we pass our trapping beam through an electro-optic 
modulator (EOM) which then produces the repump frequency. The operation of the EOM will be discussed shortly. For now, the EOM allows us to use only one main laser for both the trapping and repump beams. Finally, we also have a Toptica DL100 external cavity diode laser with nominal output of $20 \mathrm{~mW}$, which also operates at the repump frequency. This laser provides repump light prior to loading our magnetic trap, where as we shall see the main repump beam is difficult to use. We have also used it prior to probing the atoms, depending on the state in which they are magnetically trapped. Again, the details are described later in this chapter.

In order to modulate laser frequency and achieve rapid switching times, we used two devices, the acousto-optic modulator (AOM) and the electro-optic modulator (EOM). Both devices are purchased commercially, although we built our own drive electronics. I will now consider each in turn.

We use acousto-optic modulators, or AOMs, primarily to control beam intensity, but also to make small modifications of beam frequency $(\sim 100 \mathrm{MHz})$. The AOM contains an optically transparent material to which a piezo-electric transducer is bonded. The piezo is driven with an RF signal generator, producing traveling acoustic waves in the glass. Acoustic waves are longitudinal, transmitted via low amplitude spatially periodic density variations in the glass. The acoustic waves also induce comparable variations in the index of refraction of the glass medium. Pursuing this line of reasoning, the AOM becomes a sinusoidal diffraction grating as depicted in Figure 2.2. While this is a useful device for understanding the effect of an $\mathrm{AOM}$ on a light beam, a more convenient model considers the collision process between photons and phonons. Following the conservation 


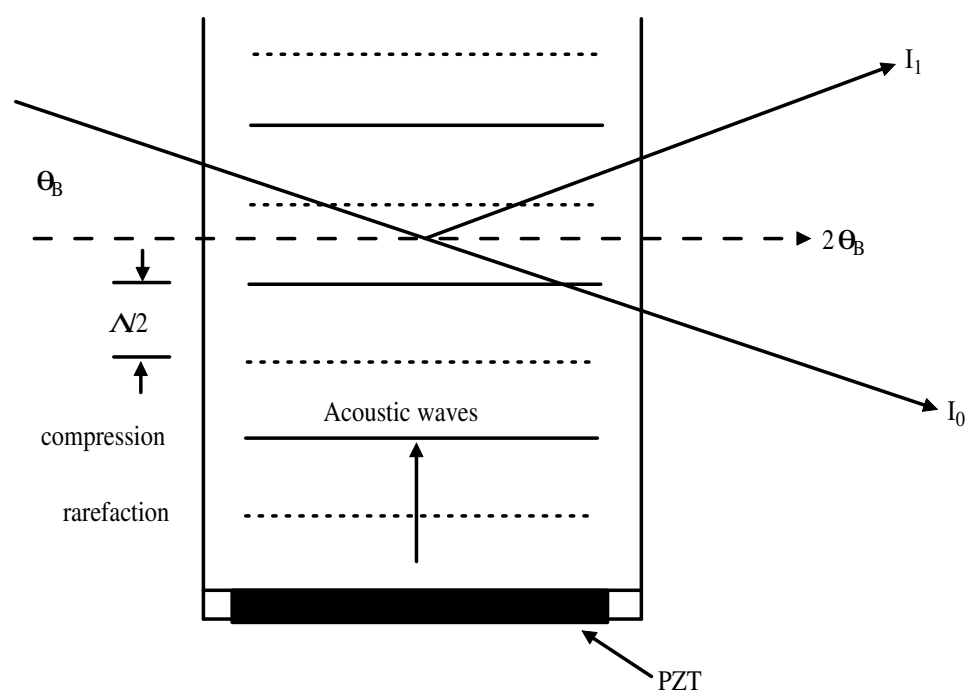

Figure 2.2: Diagram of an acousto-optic modulator. The PZT is driven with an RF signal, and produces acoustic waves that travel through the optically transparent medium, often glass. The acoustic waves are longitudinal with wavelength $\Lambda$, causing the glass to compress and rarefy slightly as each sound wave passes. These spatially periodic density variations are accompanied by variations in the index of refraction, creating a Bragg diffraction grating. The deflection angle of the frequency-shifted output beam is on the order of several milliradians.

of momentum, we find

$$
\hbar k_{ \pm 1}=\hbar k_{0} \pm \hbar K
$$

where $k_{ \pm 1}$ is the wave vector of the scattered light, $k_{0}$ the wave vector of the incident light, and $K$ the wave vector of the sound wave. If we divide out the $\hbar$ 's and consider only the $m=+1$ diffracted order, we have

$$
k_{+1}=k_{0}+K
$$

Similarly, application of the conservation of energy to the photon-phonon collision yields

$$
\omega_{+1}=\omega_{0}+\Omega
$$



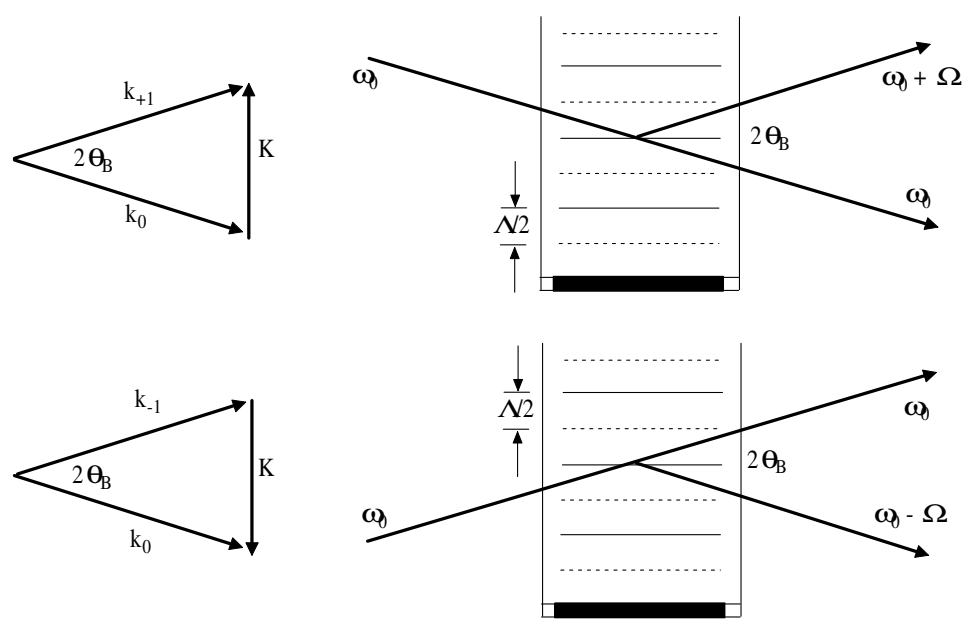

Figure 2.3: Wave vector diagrams for photon-phonon collisions in an AOM. The $\mathrm{m}=+1$ diffraction order is Bragg diffracted up, while the $\mathrm{m}=-1$ order is Bragg shifted down. The wavelength of the phonon is much greater than the wavelength of the photon, so that $K \ll k_{0}$ and the vector diagrams are very nearly isosceles triangles. As a result, the geometry is satisfied by a critical angle $\theta_{B}$, known as the Bragg angle.

where $\omega_{+1}$ is the frequency of the scattered light, $\omega_{0}$ the frequency of the incident light, and $\Omega$ the frequency of the acoustic wave. As seen from the wave vector diagrams in Figure 2.3, these parameters are related by the Bragg angle $\theta_{B}$, such that

$$
\theta_{B}=\arcsin \left(\frac{K}{2 k_{0}}\right)=\arcsin \left(\frac{\lambda}{2 \Lambda}\right),
$$

where $\lambda$ is the wavelength of the incident light and $\Lambda$ is the wavelength of the acoustic wave.

We use several model 3080-122 AOMs from Crystal Technology Inc., with tellurium dioxide as the $\mathrm{AO}$ medium, as switches on our main laser beams. We send the first diffracted order beam from each $\mathrm{AOM}$ into our experiment, so that the AOMs activate the laser light used to cool and trap the atoms. We observe $80-90 \%$ of the incident light converted into the first diffracted order, and switching times less than $1 \mu \mathrm{s}$. To reduce 
the power in the diffracted beam we simply lower the amplitude of the RF driver, so that fewer phonons are generated. When the RF power is reduced to zero, all of the incident power should transmitted into the zero order beam with no deflection or frequency shift. However, in reality the power in the first order beam is not entirely eliminated. We have observed as little as $10^{-4}$ and as much as $10^{-2}$ of the incident light still transferred to the first order beam even when the RF power is turned off. This leakage light is weak compared to the total incident beam power, but can be significant if the incident beam is strong. For example, a $500 \mathrm{~mW}$ beam, such as our main cooling beam, can leak about $5 \mathrm{~mW}$ even when it is supposed to be off. As we will later see, the presence of even a relatively low power beam can be catastrophic for magnetically trapped atoms when the frequency of the light is near an atomic resonance. As a result, our AOM's are used in conjunction with a mechanical shutter. While the $\sim 30 \mathrm{~ms}$ switching time of the shutter is much slower than the AOM, its extinction ratio (leakage light compared to incident light) is zero, ensuring that no light is transmitted to our atoms.

We also use an AOM from IntraAction Corporation to exert fine control over laser frequency. As we saw in Equation 2.4, the angle of the exit beam varies with the wavelength of the acoustic wave. Therefore, if we change laser frequency by varying phonon frequency, we will have to realign the exit beam to the experiment. In the case of our Intraction $\mathrm{AOM}$, we circumvent this inconvenience by passing the beam through the AOM twice, so that the deflections cancel out. Specifically, the first diffraction order transmitted beam is passed to a corner cube, which reflects the beam back along the same path but displaced vertically. A lens is used to compensate for the vertical displacement. The 
beam is deflected by $+\theta$ on the first pass, and $-\theta$ on the second pass, so that the exit beam has no net deflection. Thus the angle of the exit beam is no longer dependent upon the frequency of the phonons. This double-pass AOM was purchased with a driver, which responds to an applied voltage by varying phonon frequency according to the relationship

$$
\nu=\nu_{0}-20.38 \frac{\mathrm{MHZ}}{\mathrm{V}} \times V_{\text {applied }}
$$

per pass. An applied voltage of $0.1 \mathrm{~V}$, for example, changes the frequency of the exit beam by $-4 \mathrm{MHz}$. The center frequency for this AOM is $40 \mathrm{MHz}$, with a range of $\pm 10 \mathrm{MHz}$, per pass. It is located early in the beam path so that it can be used to adjust the frequency of all beams in the experiment.

The other modulation device in our experiment is an EOM. As mentioned above, our experiment requires that two atomic transitions be driven simultaneously. Our EOM is used to supply the "repump" frequency. Its operation is based on the electro-optic (or Pockels) effect, wherein a crystal becomes birefringent in the presence of an applied voltage. The applied field induces a temporal periodicity in the index of refraction $n$ of the crystal, as opposed to the spatial periodicity of $n$ in the AOM. As a result, there is no need to discuss conservation of momentum. The incident beam undergoes a frequency shift in order to conserve energy, but is not deflected.

The modulator contains a $\mathrm{LiNbO}_{3}$ crystal, which is sometimes doped with $\mathrm{MgO}$ in order to prevent photorefractive damage that can result from overexcitation of the crystal. Our EOM, a New Focus model 4851 Visible Phase Modulator, contains a doped crystal. 


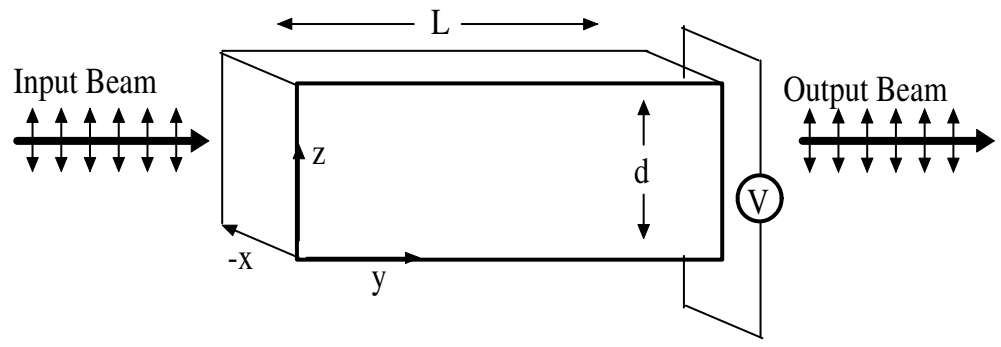

Figure 2.4: Sketch of optical beam passing through an electro-optic modulator. The polarization of the incident beam is aligned with the axis of the crystal. An RF signal generator excites photons which also travel along the crystal axis, transverse to the optical beam.

These crystals have become widely used in such applications because they display wide optical transparency windows, large electro-optic coefficients, and low RF losses. An RF signal generator supplies photons which travel through the crystal transverse to the direction of optical propagation. As shown in Figure 2.4, the optical beam is aligned so that its polarization is also along the axis of the crystal. The RF photons excite time dependent variations in $n$, resulting in a time dependent phase shift $\Delta \phi$ in the incoming beam. The induced phase shift causes power to be distributed in sidebands at a given frequency from the carrier light, according to the electro-optic effect. If the input beam has frequency $\omega_{0}$ and the RF photons have frequency $\Omega$, then the output beam is composed of photons of different colors, with frequencies $\omega_{0} \pm n \Omega$ for integer $n$. The amount of power in each of the nth-order sidebands is given by $\left[J_{n}(\Delta \phi)\right]^{2}$, where $J_{1}$ is the Bessel function of order one.

The amount of power in the carrier beam is given by $\left[J_{0}(\Delta \phi)\right]^{2}$, where $J_{0}$ is the Bessel function of order zero. The maximum power transferred to each of the first-order sidebands is observed to be 10 percent for our experiment, with the output power limited 
by the RF power level. The frequencies of the two sidebands depend on how the crystal is driven. We use at microwave driver at $6.6 \mathrm{GHz}$. The frequency is chosen in order to drive the $F=1 \leftrightarrow F^{\prime}=2$ transition of ${ }^{87} \mathrm{Rb}$, shown in Figure A.2. Accordingly, since the energy splitting between the two ground states in $6.835 \mathrm{GHz}$, the energy splitting between the excited states $F^{\prime}=3$ and $F^{\prime}=2$ is $267 \mathrm{MHz}$, and as we shall soon see the main lasers are detuned $-20 \mathrm{MHz}$ from the $F=2 \leftrightarrow F^{\prime}=3$ transition, the appropriate frequency for the EOM driver is $6.835-.267+.020=6.588 \mathrm{GHz}$.

The electronics that drive these devices were mostly built in our lab, and their circuit diagrams are included in Appendices B and C. All of these drivers were designed to accept an input voltage from a computer-driven pulsing system. Our BEC experiment requires precise control over the timing of each stage in the process of making a condensate, and so we have a dedicated pulsing system which accepts a user-written pulse sequence and executes it on command. The heart of our pulse system is an ADwin- $4 \mathrm{~L}$ real time analog/digital control board. Using the ADwin system, we can apply control voltages for time scales as small as $1 \mu \mathrm{s}$.

\subsection{Laser Frequency Stabilization}

Ideally a laser would operate at a fixed frequency without external assistance. Many atomic physics experiments rely on laser light with little or no frequency fluctuations. However, there are certainly many factors that affect laser stability. These include temperature drift, magnetic field drift, mechanical stress, etaloning and birefringence in the optical components, laser intensity noise, laser alignment fluctuations, and electronic off- 
sets. To compensate for these drift mechanisms, lasers are often frequency stabilized by means of an external lock.

I will discuss two methods of laser frequency stabilization, or laser locks. The first method is the saturated absorption lock, which has become the workhorse of laser locks. The second is a relatively new technique known as the Dichroic Atomic Vapor Laser Lock (DAVLL). The merits of both techniques will be described, as well as the work I did to improve the performance of our DAVLL.

\subsubsection{Saturated Absorption Lock}

Saturated absorption spectroscopy is an extremely reliable method to ascertain laser frequency. The laser is shone through a glass cell containing an atomic vapor. Laser frequency is obtained through analysis of the light transmitted through the cell. Saturated absorption is particularly useful because of the narrow spectral features obtainable, often about $10 \mathrm{MHz}$ full width half max.

To determine laser frequency, the saturated absorption lock exploits the strong frequencydependence of atomic absorption. As laser frequency is swept through the atomic resonance, the absorption grows rapidly (and conversely, transmission through the vapor cell falls) in a Gaussian-shaped profile with the peak of the Gaussian at the resonance frequency. If the atoms were stationary along the beam path, the absorption profile would be a narrow Lorentzian function on the resonance. However, the motion of the atoms is in fact random, with velocity components along the path of the laser beam. Due to the Doppler effect, the frequency of the light is shifted as $\omega_{-}=\omega_{0}-k v$ for atoms mov- 
ing parallel to the beam, and $\omega_{+}=\omega_{0}+k v$ for atoms moving antiparallel to the beam. Although there are many mechanisms for broadening the absorption profile, such as collision broadening, power broadening and transit time broadening, Doppler broadening is the primary mechanism in a dilute gas.

While a Doppler broadened profile is commonly hundreds of $\mathrm{MHz}$ wide, we often would like to ascertain laser frequency to within a few MHz. This can be accomplished by exploiting the phenomenon of saturation. The rate at which an atom scatters photons is

$$
R_{s}=\frac{\Gamma}{2}\left(\frac{\frac{I}{I_{s a t}}}{1+\frac{I}{I_{s a t}}+4 \frac{\Delta^{2}}{\Gamma^{2}}}\right)
$$

where $I$ is the total incident radiation intensity, $\Delta$ is the detuning of the laser light from resonance, $\Gamma$ is the natural linewidth, and $I_{\text {sat }}$ is the saturation intensity. Table A.4 shows saturation intensities for ${ }^{87} \mathrm{Rb}$ and various light polarizations. As laser detuning is decreased, the $\Delta$ term approaches zero. As laser intensity is increased, the scattering rate $R_{s}$ approaches a maxiumum of $\Gamma / 2$.

In saturated absorption spectroscopy, two weak probe beams travel through a vapor cell. A counterpropagating pump beam, which is at approximately saturation intensity, crosses one of the probe beams as it passes through the vapor cell as shown in Figure 2.5. As laser frequency is swept, each probe beam exhibits a Doppler-broadened absorption profile. The counter-propagating pump beam will also exhibit a Doppler profile, but with an important difference: atoms moving parallel to the pump beam are moving antiparallel to the probe beams. Therefore, the pump and probes do not interact with the same population of atoms at the same time. The exception is the group of atoms with zero 


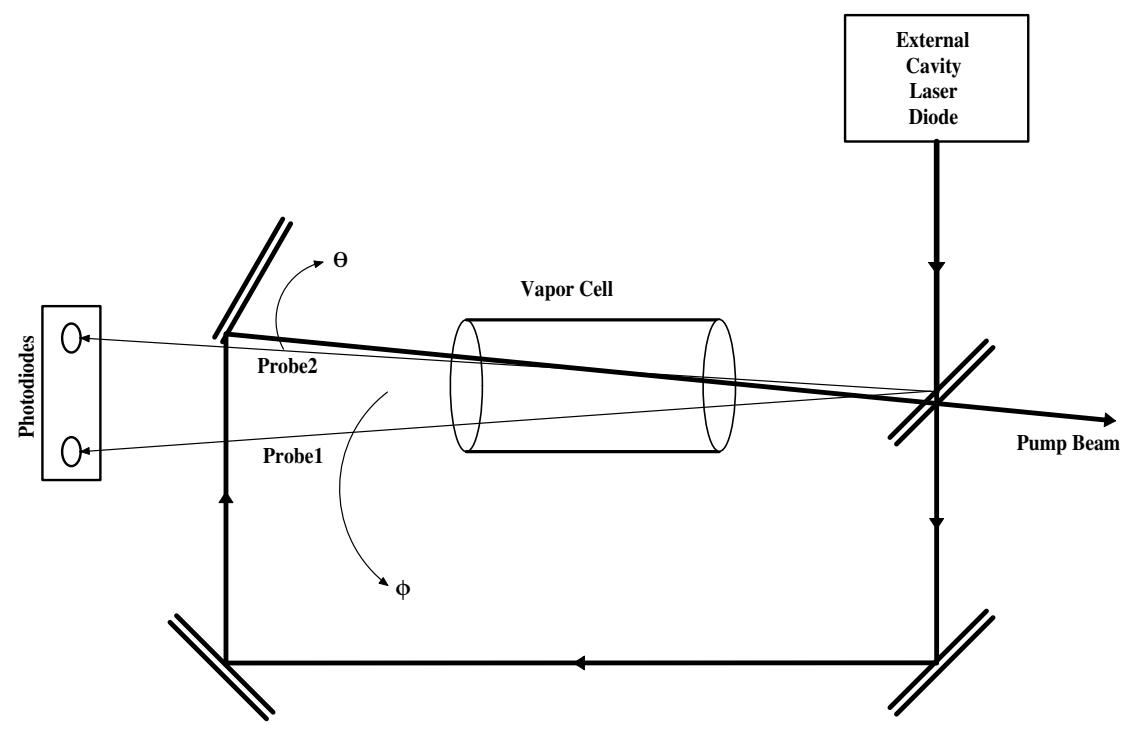

Figure 2.5: Laser beam path in saturated absorption spectroscopy. The input beam is sent to a wedge, which reflects two weak beams with an angle $\phi$ between them, and transmits a stronger beam. The weak probe beams pass directly through the vapor cell, to the photodiodes. The strong pump beam is carried around the cell, and enters from the opposite side so that it opposes the probe beams. The pump crosses only one of the probes while traversing the vapor cell. The saturated absorption signal is optimized when the angle $\theta$ between pump and probe is minimized.

velocity along the beam paths. These atoms will interact simultaneously with pump and probe beams. The pump beam is strong enough to saturate the atoms, so that they will absorb very little light from the weaker probe beam. The sudden drop in absorption results in a sharp, Doppler-free feature at the resonant frequency. This peak sits on top of the Doppler profile, but by subtracting this signal from the other probe beam, we can isolate the Doppler-free feature, and produce the saturated absorption signal.

Figure 2.6 illustrates the absorption profiles of two probe beams in a generalized twolevel atomic system. The first probe is not crossed by the pump beam. It exhibits a normal Doppler-broadened signature. But the second probe beam crosses the pump beam while traveling through the vapor cell. Atoms with zero velocity along the beam path will 

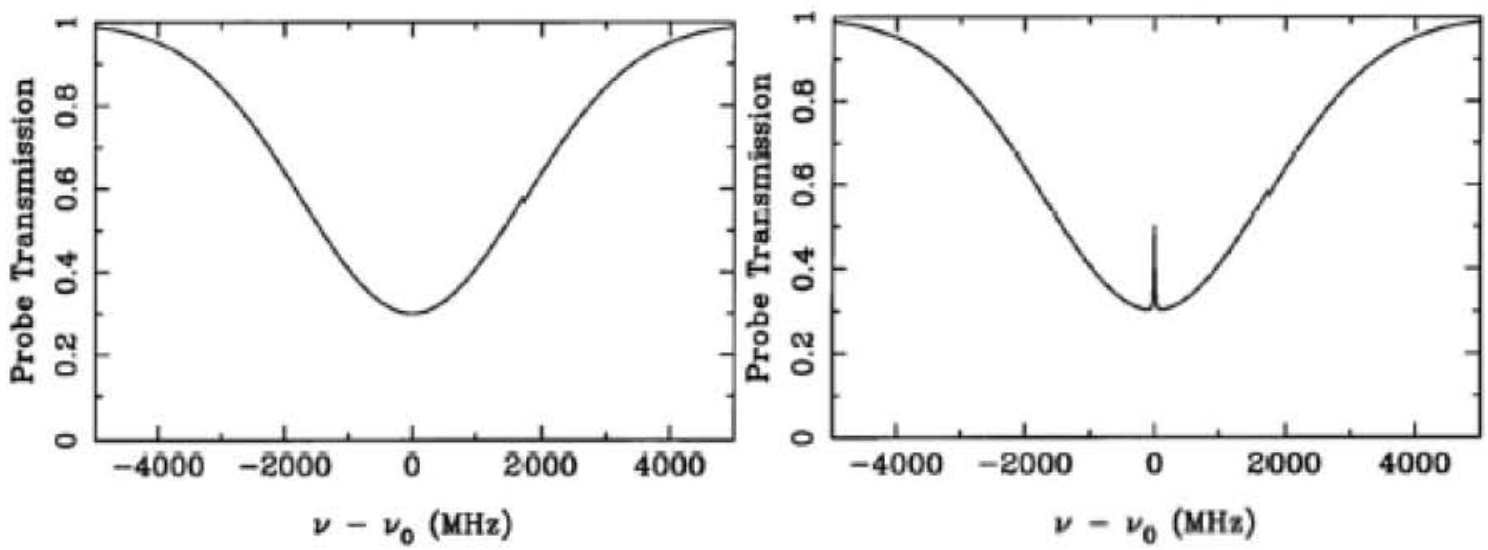

Figure 2.6: Example of saturated absorption in a two-level system. Each graph is the absorption profile of a probe beam. The first probe does not cross the pump beam, so it exhibits a normal Doppler-broadened profile. The second probe crosses the pump while traveling through the vapor cell, resulting in a sharp increase in probe transmission at the atomic resonance. By subtracting one signal from the other, we isolate the Doppler-free feature and create a saturated absorption trace. Images from California Institute of Technology [2].

interact simultaneously with probe and pump, resulting in an increase of transmission of the probe beam at the resonance frequency This sharp peak is the saturated absorption feature. By subtracting one signal from the other, we isolate the Doppler-free feature.

In a multi-level atom, the saturated absorption spectrum can be more complicated. Take for example an atom with one ground state and two closely spaced excited states, as shown in Figure 2.7. The first feature occurs at $\nu_{1}$, the frequency of the transition between the ground state and the first excited state. The third feature occurs at $\nu_{2}$, the frequency of the transition between the ground state and the second excited state. The middle feature occurs at $\left(\nu_{1}+\nu_{2}\right) / 2$, and is called a "cross-over resonance." This peak arises because there is a group of atoms with velocity such that the Doppler shift brings the pump into resonance with one transition and the probe into resonance with the other. These atoms can interact with either beam, so that the observed feature is at the average 


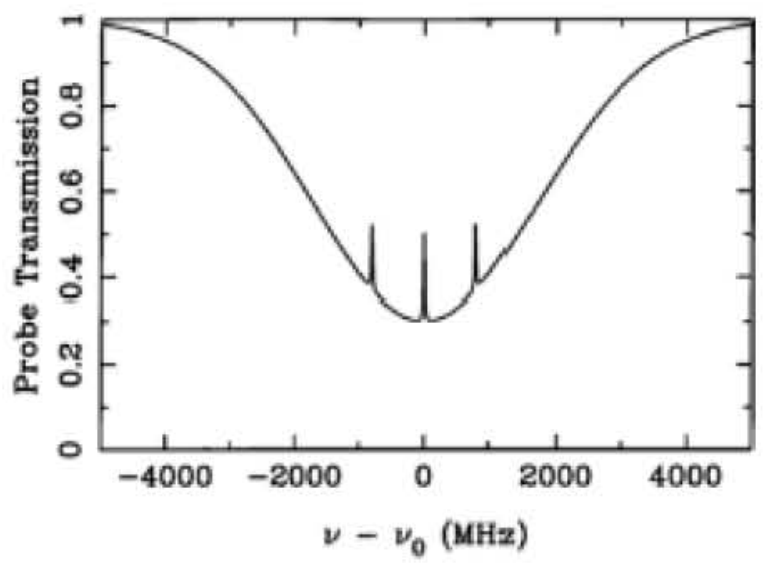

Figure 2.7: Example of saturated absorption in a multi-level system. The first feature occurs at $\nu_{1}$, the frequency of the transition between the ground state and the first excited state. The third features occur at $\nu_{2}$, the frequency of the second excited state. The middle feature occurs at $\left(\nu_{1}+\nu_{2}\right) / 2$, the average of the frequencies of the two excited states, because atoms in the proper velocity group find the pump beam Doppler-shifted to one transition and the probe beam Doppler-shifted to the other. Image from California Institute of Technology [2].

of the two frequencies. Furthermore, in an atom with several excited states there can be many types of crossovers, but that subject is beyond the scope of this dissertation. Detailed discussions can be found in $[8,37,45-47,75]$, among others.

A saturated absorption signal can be used to lock the laser to the resonance frequency. However, some additional measures must be taken to make a reliable lock. If we just use the saturated absorption feature as is, our lock will be unable to discern the sign of the correction needed to keep the laser on resonance. The signal diminishes on either side of the peak, and so simply examining the level of absorption does not reveal to which side of the peak the laser has drifted. This problem is corrected through differentiation of the saturated absorption signal, creating a dispersive-shaped curve that passes through zero at the resonance frequency. By locking to the zero crossing, we fix the laser on resonance. If the laser frequency drifts red, the error signal will become increasingly positive, whereas 
if the laser drifts blue the error signal becomes more negative.

The saturated absorption lock provides a stable, high resolution frequency reference. The optics involved are simple, but the electronics can be quite involved. A lock-in amplifier is often required to perform the differentiation. We constructed our own lockin, and the circuit diagram is included in Appendix D. A method of modulating the saturated absorption signal must be introduced. One common method is to modulate laser frequency. We used another technique whereby the Zeeman splittings of the atomic energy levels are modulated. Our vapor cell was encased within a solenoid of currentcarrying wire, and the current through the solenoid was driven by a function generator with a $1 \mathrm{kHz}$ sine wave. The resultant magnetic field was oriented along the axis of the vapor cell, with periodic magnitude.

Although our saturated absorption lock does require a good deal of control electronics, its performance is robust. Our laser frequency is stable to within about $1 \mathrm{MHz}$ per day. Our Ti-Sapphire was locked to the crossover between the $\left|F=2 \leftrightarrow F^{\prime}=3\right\rangle$ and $\left|F=2 \leftrightarrow F^{\prime}=2\right\rangle$ transitions of ${ }^{87} \mathrm{Rb}$ via a saturated absorption lock. Our experiment was centered around the $|2 \leftrightarrow 3\rangle$ transition, but because of frequency offsets due to our $\mathrm{AOM}$ switches we found it most convenient to lock to this crossover which is $133.5 \mathrm{MHz}$ red of the primary transition. Figure 2.8 shows the laser beam path into the experiment, along with all frequency shifts. If we sum all frequency shifts, we find that at the atoms in the experiment, laser frequency is $+115.5-133.5-84+80=-22 \mathrm{MHz}$. By adjusting the offset on the lock-in amplifier, we can move the lock point along the width of the dispersion-shaped lock signal. Since our lock signal had some extra structure that left a 


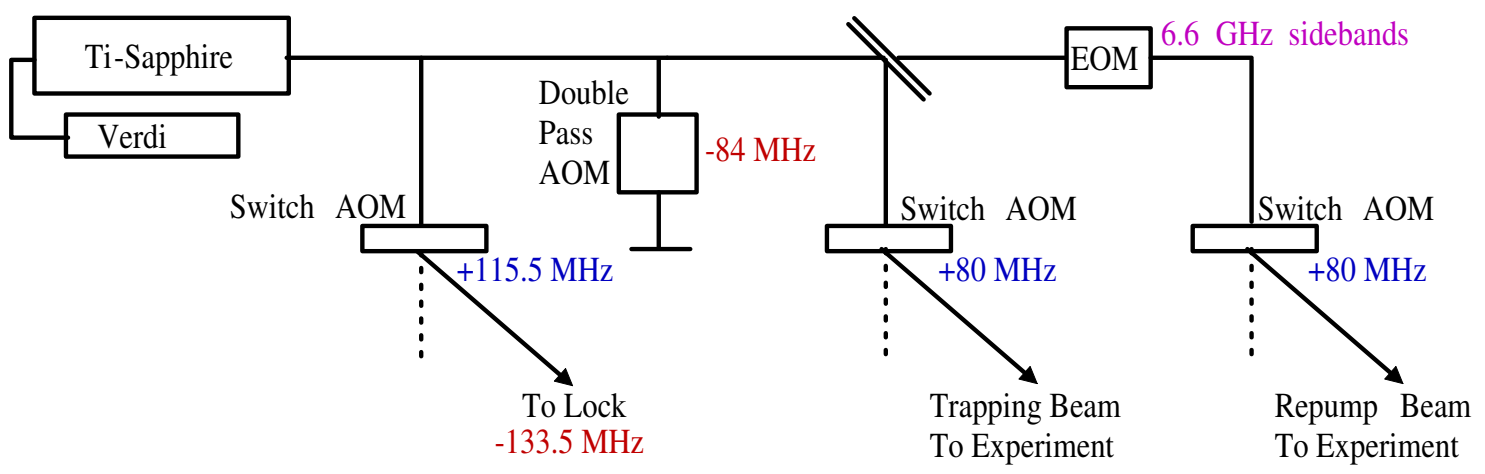

Figure 2.8: Laser beam path into experiment. A Ti-Sapphire MBR-110, pumped by a $10 \mathrm{~W}$ Verdi, provides the main laser beam for our experiment. Immediately after the light exits the laser cavity, we send a small fraction of the total power into a switch AOM at $+115.5 \mathrm{MHz}$ and then to a saturated absorption lock system. The rest of the light proceeds to a double pass AOM, where laser frequency can be adjusted by up to $50 \mathrm{MHz}$ per pass. We then split the beam into two separate paths, to create the trapping and repump beams. The trapping beam passes through a switch AOM at $+80 \mathrm{MHz}$ and then directly to the experiment. The repump beam first encounters an EOM, which adds 6.6 GHz sidebands to the carrier frequency. The repump beam then passes through another switch AOM, also at $+80 \mathrm{MHz}$, and then to the experiment.

section of the signal undesirable for locking, we ended up adjusting the offset until the lock was observed to behave reliably. This resulted in slight displacement from the peak of the crossover, so that we operated our experiment with laser detuning of about $20 \mathrm{MHz}$ red of the $F=2 \leftrightarrow F^{\prime}=3$ resonance.

Although the saturated absorption lock is probably the most widely used laser lock, it is not the only lock available. As we shall now see, one recent innovation has been the Dichroic Atomic Vapor Laser Lock, which is slowly gaining popularity in the atomic physics community. We used a saturated absorption lock on our Ti-Sapphire laser, but we also used a DAVLL for our diode laser and in that process became interested in the stability of this lock. 


\subsubsection{Dichroic Atomic Vapor Laser Lock}

The Dichroic Atomic Vapor Laser Lock (DAVLL) has been demonstrated in He [17], Rb [19], Cs [11, 18,49], and K [3] as an alternative to standard techniques for laser frequency stabilization based on saturated absorption [23]. However, the DAVLL uses the entire Doppler-broadened profile, which allows for greater tunability and capture range. These features make the DAVLL especially promising for applications in industrial and field environments. A variety of other simplified saturated absorption techniques have also been proposed $[50,51,56,64,67]$, but none of these matches the DAVLL's capture range and tunability. We were interested in using the DAVLL in our experiment because of the large tuning range, since our atom interferometer ultimately requires a far-off resonant laser beam as mentioned in Chapter 4.

In order for the DAVLL to realize its promise, however, it must reliably hold the laser to one frequency. Both Corwin et al. and Beverini et al. have observed good stability, with better than $500 \mathrm{kHz}$ peak-to-peak variation over many hours. In contrast, our initial attempts to implement a DAVLL exhibited much larger fluctuations. Our investigations led us to conclude that ambient temperature fluctuations were a dominant contributor to instability in our system. I will discuss the temperature-dependent characteristics of the DAVLL, and show how we have significantly reduced the effects of temperature on the lock frequency.

The DAVLL method was clearly described by Corwin et al. [19]. It consists of a vapor cell placed in a longitudinal magnetic field, followed by a quarter-wave retarder and a polarizing beamsplitter, as shown in Figure 2.9. The light entering the cell is linearly 
polarized and can be considered as a superposition of $\sigma^{+}$and $\sigma^{-}$light. In the presence of the magnetic field, the absorption profiles of the $\sigma^{+}$and the $\sigma^{-}$light are shifted to higher and lower frequencies, respectively. The retarder and polarizing beamsplitter separate the circular components, which are measured with two photodiodes. The difference between the two signals is a dispersion-like curve as a function of frequency, which provides an error signal for the lock. The width of the signal is on the order of the Doppler width, $\Delta \nu_{d}$. Our testing scheme used a DAVLL with Rb atoms, locked to the ${ }^{87} \mathrm{Rb} S_{1 / 2} F=2 \leftrightarrow$ $P_{3 / 2} F^{\prime}=3$ transition, although in our BEC experiment we actually used the DAVLL to lock the diode laser to $F=1 \leftrightarrow F^{\prime}=2$ transition (repump frequency). We note that a variation on the DAVLL which is optimized for large detunings is described by Yashchuk et al. [74]. We have not studied this method extensively, but we have implemented it and observed frequency drifts similar to those discussed here.

As in any servo system, the laser is held at a frequency where the error signal is zero. This frequency (the "lock point") can be adjusted by introducing an offset to the dispersion signal. In this way, the entire $\Delta \nu_{d}$ range is available for locking. The offset can be adjusted in several ways, including rotating the quarter-wave plate, introducing an optical attenuator in one of the beams, or applying an electronic offset to the error signal. We use an optical attenuator consisting of a rotatable polarizer. The polarizer is difficult to adjust with sufficient precision, so we also attenuate the beam with a tilted glass window. By making small adjustments to the tilt angle, very fine control can be achieved.

We found, as noted by Corwin et al., that the first step in achieving good stability with 


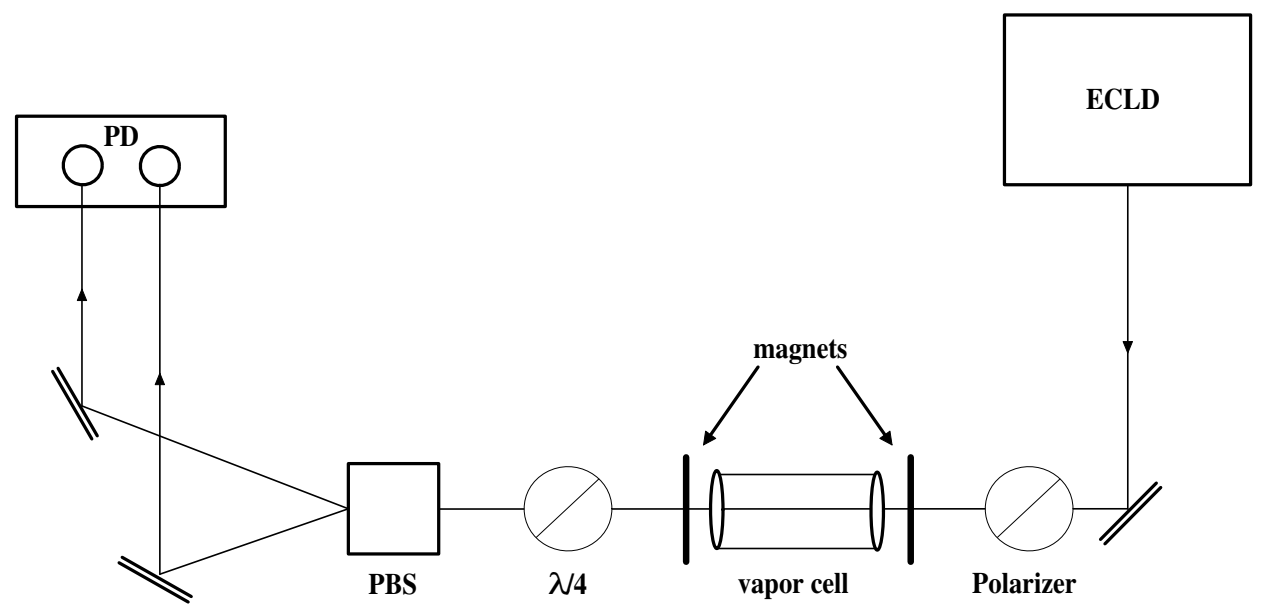

Figure 2.9: Optical path in DAVLL system. The input beam passes through a polarizer to the vapor cell. The magnets on either side of the cell supply a longitudinal magnetic field, allowing the linearly polarized input beam to be decomposed into a linear combination of circular polarizations. The beam then passes through a quarterwave plate, which converts the circular components to linear polarizations. The polarizing beam splitter separates the two linear polarizations, which are then passed to two separate photodiodes. The difference between the photodiode signals is the characteristic DAVLL signal.

the DAVLL is to use good polarizers. We used calcite polarizers in all cases. In contrast, using dichroic polarizing film or dielectric polarizing beamsplitter cubes caused frequency drifts as large as tens of $\mathrm{MHz}$ per hour. For the retarder, we used both a Fresnel rhomb and a zero-order quarter-wave plate with success.

Even with good polarizing optics, we still initially saw day-to-day variations in the lock point of up to $10 \mathrm{MHz}$, which is inadequate stability for high-precision experiments. Such fluctuations also limit applications of the DAVLL, since the small adjustments needed to correct for this drift would not be possible in a field-deployed device. In an effort to eliminate the fluctuations, we put extra care into the alignment of the optics. We precisely aligned the axis of the polarizer to the axis of the polarizing beamsplitter, and set the quarter-wave plate axis to $\pm 45^{\circ}$ as accurately as possible. We then used the optical 
attenuator to eliminate the residual offset in the error signal, so that far from resonance the signal was zero. Under these conditions, day-to-day repeatability of about $1 \mathrm{MHz}$ was achieved.

Unfortunately, with this alignment, the lock point of the system was about $100 \mathrm{MHz}$ red of the desired transition to the $F^{\prime}=3$ excited state. When we reintroduced an offset to tune the lock point back, the stability again deteriorated. We eventually attributed this effect to a variable sensitivity to temperature fluctuations. An important source of temperature sensitivity is the density of the atomic gas, as first noted by Beverini [11]. At room temperature, the density of Rb gas varies by about $10 \%$ per $\mathrm{K}$.

Changes in density will affect the lock frequency only if the zero crossing of the DAVLL signal is shifted, which happens if the absorption of the $\sigma^{+}$and $\sigma^{-}$polarizations change by different amounts. Naively, this does not occur when locked to the very center of the Doppler profile, because in this case the two polarizations are absorbed symmetrically. However, when the lock point has been displaced with an offset of some kind, the symmetry is lost and temperature dependence is introduced. For instance, if the $\sigma^{-}$beam is attenuated, then the lock point will shift to a higher frequency where the net transmissions for $\sigma^{+}$and $\sigma^{-}$are again the same. If the temperature subsequently rises, the atomic absorption of both components will rise by the same fractional amount but the external attenuation will remain constant. Accordingly, the difference between the net transmissions will change, and the lock point will vary. Similar effects occur if the quarter-wave plate is not set at $\pm 45^{\circ}$.

In reality, understanding the temperature dependence is more complex, because the 
hyperfine splitting of the excited state is comparable to the Doppler width and the Zeeman width, and this gives an intrinsic asymmetry to the DAVLL signal. To investigate the system in more detail, we developed a computer simulation. We followed the approach described, for instance, by Clifford [18] or Beverini [11]. We model the transmission of laser light through the rubidium cell by calculating the complex transmission amplitudes for each available $\sigma^{+}$and $\sigma^{-}$transition, assuming the transitions to have Doppler-broadened profiles. The atomic eigenstates and coupling coefficients are calculated by diagonalizing the system Hamiltonian for the $5 S_{1 / 2}$ and $5 P_{3 / 2}$ manifolds, including hyperfine and Zeeman effects. Both the ${ }^{87} \mathrm{Rb}$ and ${ }^{85} \mathrm{Rb}$ isotopes are included, since their transitions overlap somewhat. The DAVLL signal is then calculated in terms of the angle $\theta$ of the transmission axis of the initial polarizer and the angle $\phi$ of the fast axis of the quarter-wave plate. Both angles are measured counter-clockwise from horizontal, as observed from the output side of the optic. Our magnetic field is directed opposite to the propagation vector of the light.

We used Jones representations for the optics in the system. The light transmitted through the input polarizer has polarization

$$
V_{\text {in }}=\left[\begin{array}{c}
\cos \theta \\
\sin \theta
\end{array}\right]
$$

The transmission of light through the vapor cell is represented as

$$
M_{\text {cell }}=\left[\begin{array}{cc}
1 & 1 \\
i & -i
\end{array}\right]\left[\begin{array}{cc}
t_{+} & 0 \\
0 & t_{-}
\end{array}\right]\left[\begin{array}{cc}
1 & -i \\
1 & i
\end{array}\right]
$$


where $t_{+}$and $t_{-}$are transmission coefficients for $\sigma^{+}$and $\sigma^{-}$light, respectively, and are discussed further shortly. The quarter-wave plate matrix is

$$
M_{\lambda / 4}=\left[\begin{array}{cc}
\cos \phi & -\sin \phi \\
\sin \phi & \cos \phi
\end{array}\right]\left[\begin{array}{cc}
1 & 0 \\
0 & e^{i \alpha}
\end{array}\right]\left[\begin{array}{cc}
\cos \phi & \sin \phi \\
-\sin \phi & \cos \phi
\end{array}\right]
$$

where $\alpha \approx 90^{\circ}$ is the birefringence of the wave plate. The output polarization is determined by

$$
V_{\text {out }}=M_{\lambda / 4} M_{\text {cell }} V_{\text {in }} \equiv\left[\begin{array}{c}
H \\
V
\end{array}\right]
$$

in order to find the relative strengths of the two polarizations exiting the DAVLL system. Finally, to model our optical attenuation we included a factor $\rho$ resulting in an output signal $S=|V|^{2}-\rho|H|^{2}$.

The transmission coefficients $t_{+}$and $t_{-}$are calculated using

$$
t=\exp \left(-\frac{A}{2}+i \beta\right)
$$

with amplitude $e^{-A / 2}$ and phase $\beta$. The amplitude and phase are obtained by summing over allowed transitions $j \leftrightarrow k$

$$
\begin{aligned}
A & =\sum_{j k} A_{j k} \\
\beta & =\sum_{j k} \beta_{j k}
\end{aligned}
$$


For each transition,

$$
\begin{gathered}
A_{j k}(\nu)=\frac{\sqrt{\pi}}{2} \frac{\Gamma}{w} a_{j k} \exp \left[-\left(\frac{\nu-\nu_{j k}}{w}\right)^{2}\right] \\
\beta_{j k}(\nu)=-\frac{\sqrt{\pi}}{2} a_{j k} F\left(\frac{\nu-\nu_{j k}}{w}\right)
\end{gathered}
$$

where $a_{j k} \equiv \sigma_{j k} n_{j} L$. Here $\sigma_{j k}$ is the unbroadened resonant scattering cross-section for the $j \leftrightarrow k$ transition, $n_{j}$ is the temperature-dependent density of Rb atoms in state $j, L$ is the length of the cell, $\Gamma$ is the natural linewidth, $w \equiv\left(\frac{2 k_{B} T}{m \lambda^{2}}\right)^{1 / 2}$ parameterizes the Doppler broadened linewidth, $T$ is the temperature, $m$ is the mass, $k_{B}$ is Boltzmann's constant, $\lambda$ is the wavelength of the laser, $\nu$ is the laser frequency, $\nu_{j k}$ is the resonant frequency, and $F$ is Dawson's integral [35].

The model neglects effects from optical pumping and saturation, and assumes the magnetic field to be uniform. With these assumptions, we calculate a model DAVLL signal and its temperature dependence.

The experimental system used a Toptica DL100 diode laser system and a $50 \mathrm{~mm}$ long Rb vapor cell from Technical Glass, Inc. For the polarizing optics, we used a GT5 polarizer and WPQ zero-order wave plate from Thor Labs, and a PR-15 Rochon polarizing beam splitter from OFR. A second Rb cell was used to make a saturated absorption measurement, which provided a frequency reference as the laser was scanned. Figure 2.10 shows the DAVLL signals obtained in the experiment and predicted by the model, along with the saturated absorption reference curve. The increasing signals at high frequencies are due to the ${ }^{85} \mathrm{Rb}$ resonance. We do not continue the plot further because nonlinearity of the laser frequency scan introduces a growing error in the frequency scale. The non- 


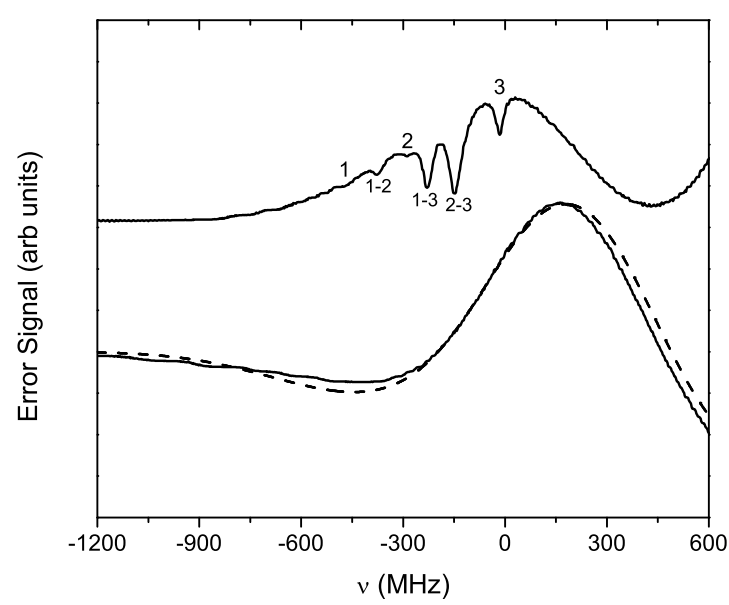

Figure 2.10: DAVLL traces. The lower solid curve shows the experimental error signal as a function of frequency for an input polarization angle of $\theta=90^{\circ}$ and a wave plate angle of $\phi=134^{\circ}$. The frequency scale is zero at the $F=2 \leftrightarrow F^{\prime}=3$ resonance, as measured using the saturated absorption trace shown by the upper curve. The saturated absorption features are labeled according to excited state, so that 3 marks the $F=2 \leftrightarrow F^{\prime}=3$ transition, and 2-3 marks the crossover between $F=2 \leftrightarrow F^{\prime}=2$ and $F=2 \leftrightarrow F^{\prime}=3$ transitions. The dashed curve is the model prediction. The amplitude of the model is adjusted to match the data, and the wave plate retardance in the model is set to $\lambda / 1.62$ in order to compensate for the retardance of the cell windows, as discussed in the text. For both the model and data, the optical attenuation was adjusted to make the signal zero far from resonance.

linearity was measured using the location of ${ }^{85} \mathrm{Rb}$ saturated absorption peaks, giving a fractional frequency error of roughly $0.05 \mathrm{GHz}^{-1}$ times the frequency detuning from the ${ }^{87} \mathrm{Rb}$ absorption lines.

Although similar, the experimental and model DAVLL traces differ in detail. Similar disagreement has been noted by other investigators $[11,18]$. One potential source of discrepancy is stray birefringence of the optical elements, in particular the cell windows. (Birefringence in the glass windows can be induced by mechanical stress.) We checked for this effect by observing the offset signal introduced by the cell to an off-resonant beam. This yielded an estimated window birefringence of about $\lambda / 20$. Understanding this birefringence in detail is difficult, since it varies with position on the window and the 
effects of the first and second windows cannot easily be isolated. We tried to account for it in the model by varying the model values of $\theta, \phi$ and $\alpha$. No adjustment of the angles was needed, but the best agreement was obtained when the wave plate retardance $\alpha$ was raised by about $\lambda / 17$, to a net value of $\pi / 1.62$. This value is used in the all the plots and analysis shown.

Another potential source of error is optical pumping. Our model assumes that all of the ground state levels are equally occupied, but as the atoms interact with the laser, the level distribution will evolve. The laser beam used had a power of $100 \mu \mathrm{W}$ and a beam waist of roughly $2 \mathrm{~mm}$, so that an atom would typically scatter about 10 photons while moving across the beam. Optical pumping effects are thus likely to be important, but incorporating them would require a considerably more sophisticated model. Since we wished to focus on stability effects, we did not pursue this question.

A third potential error is the model assumption that the magnetic field is uniform. Experimentally, the field was generated by placing the cell between two large flexible magnetic sheets with small holes cut in them for the laser beam. The resulting field varied between $105 \mathrm{G}$ and $135 \mathrm{G}$ across the length of the cell. Varying the model field across this range had little effect.

The experiment used a DAVLL system that permitted temperature control. The cell had flexible heater strips wrapped around both ends. The heaters sat on a brass mount which was water cooled and held at $20^{\circ} \mathrm{C}$. The temperature of the cell was monitored using a thermocouple taped to the glass wall. By supplying an ac current of about $50 \mathrm{~mA}$ to the heaters, the cell could be heated to $26^{\circ} \mathrm{C}$. It took about 30 minutes for the optical 


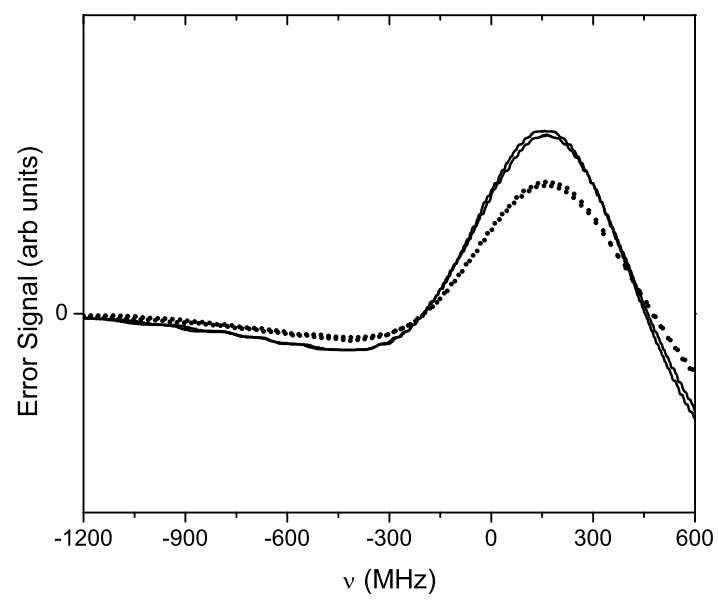

Figure 2.11: Temperature sensitivity of DAVLL error signal. The solid traces were measured at a temperature of $26^{\circ} \mathrm{C}$ and the dashed traces at $20^{\circ} \mathrm{C}$, for the same polarization settings as in Figure 2.10. Two traces are shown at each temperature.

signal to equilibrate after changing the temperature.

We measured the lock sensitivity by comparing scans such as those in Figure 2.10 at the high and low temperatures. Different scans were aligned using the saturated absorption reference signal. Figure 2.11 shows typical results obtained, in which a clear variation with temperature is evident. This indicates that the laser lock will also vary with temperature. The sensitivity of the lock point frequency $\nu_{0}$ can be estimated as

$$
\frac{\Delta \nu_{0}}{\Delta T} \equiv \frac{\Delta S}{\Delta T} \frac{1}{d S / d \nu}
$$

where $\Delta S$ is the difference between the error signals at the two measured temperatures, $\Delta T$ is the temperature difference, and $d S / d \nu$ is the average slope of the traces. The result is plotted in Figure 2.12 and shows that the temperature sensitivity can be significant. 


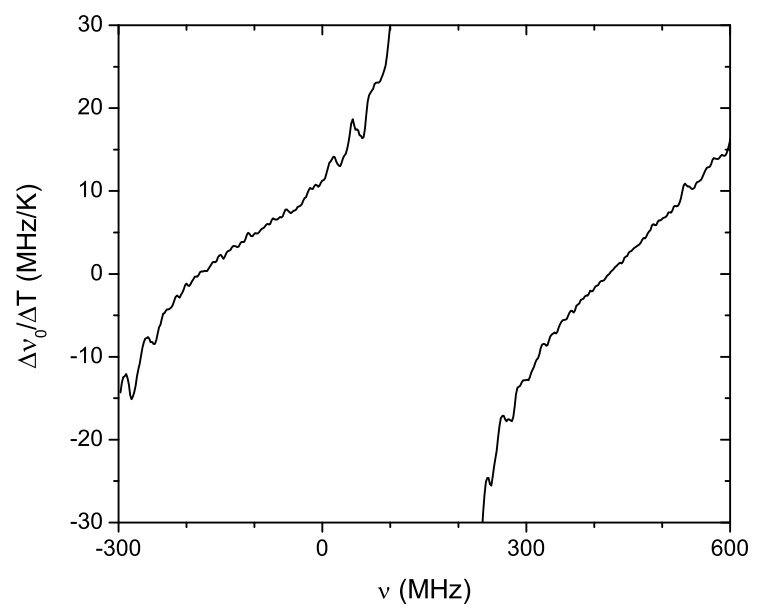

Figure 2.12: Temperature sensitivity of DAVLL lock. Using scans such as Fig. 2, the sensitivity of the laser lock frequency $\nu_{0}$ to temperature $T$ is derived, as described in the text. The temperature sensitivity diverges at the extrema of the error signal curve, here around $-400 \mathrm{MHz}$ and $+150 \mathrm{MHz}$. It is anyway not possible to lock the laser near these points because the error signal slope is too small.

Fortunately, from Fig. 2.11 and Figure 2.12 it is seen that at $\nu=-200 \mathrm{MHz}$ and $\nu=+400 \mathrm{MHz}$ the two traces cross and the temperature sensitivity vanishes. We refer to such temperature-independent points as TIPs. Operating the lock near such a TIP is desirable, as it reduces or eliminates the need for temperature stabilization. By varying $\theta$ and $\phi$, the frequency of the TIP can be set to the desired lock point. Figure 2.13 shows the measured locations of the TIPs for a range of $\phi$. It is seen that a TIP can be placed at basically any frequency where the DAVLL can be locked. In particular, with $\phi=143^{\circ}$ we obtained a measured temperature coefficient below $1 \mathrm{MHz} / \mathrm{K}$ when locking on the $F=2 \leftrightarrow F^{\prime}=3$ resonance at $\nu=0$

Figure 2.13 also shows the TIP locations predicted by the model. The model reproduces the data fairly well, except in the lower left corner of the graph the model predicts TIPs that were not observed experimentally. We suspect this is related to the fact that 


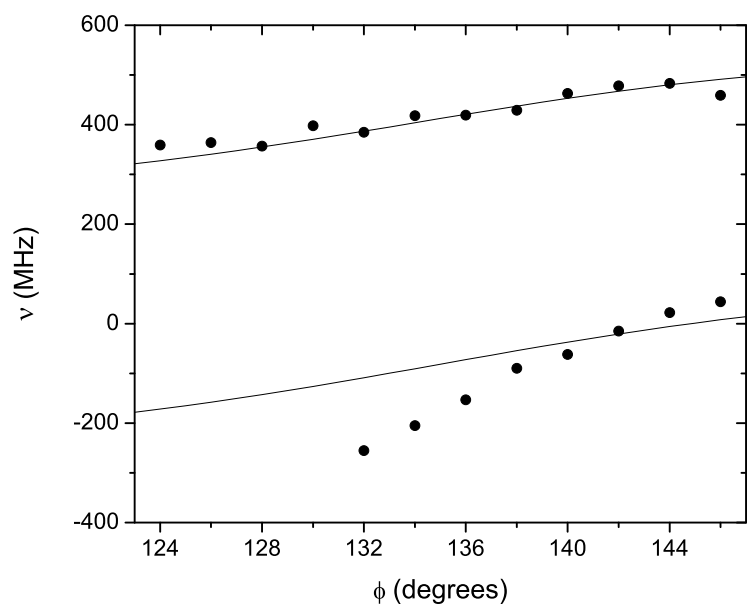

Figure 2.13: Location of temperature-independent points (TIPs). The data points indicate frequencies where the DAVLL is measured to have zero temperature sensitivity, plotted as a function of wave-plate angle $\phi$. The input polarizer angle $\theta$ was held at $90^{\circ}$ and the optical attenuation was adjusted to give zero error signal off resonance. The solid curves show the location of TIPs predicted by the model.

our model trace does not exactly reproduce the experimental trace in that region, as shown in Figure 2.10.

The location of the TIPs depends not only on the orientation of the optics, but also on the relative intensities of the two polarizations. Attenuation of one polarization relative to the other changes the shape of the DAVLL trace and moves the TIPs. So if one identifies a TIP where the error signal is nonzero, then if an attenuator is used to bring the error signal to zero the TIP location can shift. In our model and data, the attenuation was always chosen to make the error signal zero far from the resonance. For instance, in our model we identified a TIP located on resonance at $\phi=144.8^{\circ}$. This TIP was not, however, located at the zero crossing of the trace. We needed to change the attenuation factor by about $1 \%$ to bring the TIP to zero. In doing so, the TIP shifted by $0.2^{\circ}$, to 144. $6^{\circ}$. In this particular case, only a small change in attenuation was needed to bring 
the TIP onto resonance and the shift in TIP location was below the resolution of our experiment. However, this is not true for large attenuation adjustments and the effects of changing relative beam intensities should not be ignored. Using a stable electronic offset will not affect the TIP location, and might therefore be preferable.

In conclusion, the results reported here indicate that temperature fluctuations do indeed have a significant impact on the stability of a DAVLL system. This sensitivity vanishes at certain points, and it is possible to adjust the polarizer, wave plate and attenuator so that the temperature dependence is small at any desired frequency. Our numerical model supports this understanding, and demonstrates qualitative and quantitative agreement with the experimental results.

We noted previously that Corwin et al. achieved good stability without using special angles for their optics, reporting temperature coefficients of 1-2 MHz/K. [19] We believe this is because they were in fact operating near a TIP. The results reported by Corwin were for a DAVLL system locked to ${ }^{85} \mathrm{Rb}$. The excited-state hyperfine splitting of ${ }^{85} \mathrm{Rb}$ is smaller than that of ${ }^{87} \mathrm{Rb}$, so the asymmetry introduced by the hyperfine effect is reduced. This suggests that the "nominal" DAVLL set up with $\theta=0^{\circ}$ or $90^{\circ}$ and $\phi= \pm 45^{\circ}$ with no additional offset should exhibit a TIP close to the center of the Doppler profile. We confirmed this both experimentally and theoretically. In the experiment, we found a temperature coefficient at the $F=3 \leftrightarrow F^{\prime}=4$ transition below $1 \mathrm{MHz} / \mathrm{K}$ for these nominal values. We expect that a similar circumstance also explains the results of Beverini et al. for cesium.[11]

We originally planned to use a DAVLL to stabilize our Ti-Sapphire laser on the $F=$ 
$2 \leftrightarrow F^{\prime}=3$ transition, which is the main cooling and trapping transition, but when the temperature sensitivity issues arose we switched that laser to a saturated absorption lock. Our subsequent investigation of DAVLL behavior helped clarify our understanding of these issues, and we did use a DAVLL on our diode laser. Since the diode was locked to the $F=1 \leftrightarrow F^{\prime}=2$ repump transition, the results of our temperature sensitivity tests on the $F=2 \leftrightarrow F^{\prime}=3$ line were not directly applicable. However, the repump transition is less sensitive to frequency, reducing the importance of identifying a TIP.

\subsection{Laser Cooling and the Magneto-Optical Trap}

As discussed in Chapter 1, we achieve Bose-Einstein condensation when the phase space density $\rho$ is on the order of unity. The experiment begins as the MOT chamber fills with $\mathrm{Rb}$ vapor at room temperature. The phase space density of the vapor is $\sim 10^{-10}$, and the de Broglie wavelength $\lambda_{d B}$ is on the order of picometers. We lengthen $\lambda_{d B}$ and increase $\rho$ through the application laser cooling techniques as introduced in Section 1.2.

While laser cooling reduces atomic kinetic energy and cools the atoms, it does not provide spatial confinement. Laser cooling presents a viscous drag force, and the atoms slowly diffuse out of the beam path. We require an additional restoring force to collect the cooled atoms at one location. Furthermore, laser cooling loses efficiency when atomic velocity is reduced to the point where the Doppler shift no longer brings the laser frequency into resonance with the atomic energy transition. In order to continue cooling the atoms, it is necessary to compensate for the changing Doppler shift.

We address these concerns with a magneto-optical trap (MOT), a special laser cooling 


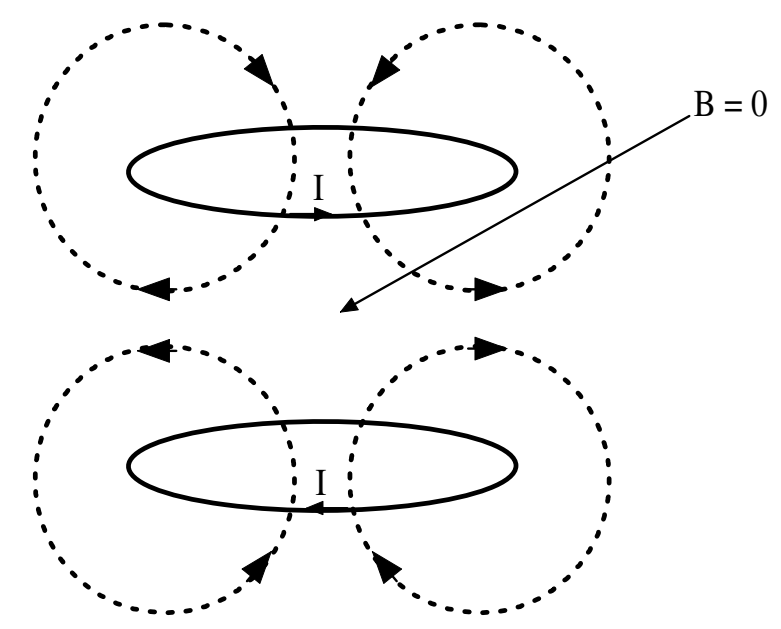

Figure 2.14: The magnetic field used in the MOT is produced by two coaxial current-carrying loops. Each loop generates field lines according to the right-hand rule. In between the loops, the opposing fields cancel each other so that there is a B field zero at the midpoint between the centers of the loops. The resultant magnetic field between the loops is a spherical quadrupole, where the magnitude of the field increases radially from the position of the field zero.

technique. First demonstrated in 1987 [55], the MOT is now widely used as a convenient source of cold trapped atoms. It relies on the combination of laser cooling and an inhomogeneous magnetic field to both cool and trap atoms. MOT's are robust, allowing significant lattitude in beam alignment, intensity balance of counterpropagating beams, and beam polarization. The modest B field gradients required are easily supplied. MOT's are therefore extremely useful and cost-efficient.

The MOT uses a spatially varying radiation force to cool atoms with various velocities and to collect the atoms at a central location. Spatial dependence is achieved through the addition of a magnetic field gradient. The appropriate magnetic field will have a zero at the center of the trap, and the field magnitude will increase with displacement from the zero. As shown in Figure 2.14, this condition is achieved with a quadrupole field generated from two current-carrying loops stacked along a common axis. 
The resulting field gradient between the two loops induces a Zeeman shift in the atomic energy levels, which varies according to the atom's location in space. The laser field is detuned from resonance so that a stationary atom located at the field zero, where there is no Zeeman shift, will weakly absorb the off-resonant radiation from either beam. Since the radiation pressure is balanced, the atom is not subject to a significant net force. If the laser beams are polarized appropriately, an atom that is displaced from the zero will undergo a Zeeman shift that brings the frequency of the atomic transition closer to resonance with the optical field opposing the atom's motion. The atom will experience a force directing it towards the zero, which is the center of the trap. With appropriate choice of B field gradient and laser detuning, the force on the atoms is similar to a spring force. The magnitude of the restoring force increases with displacement, because the rate of atomic absorption of laser photons also grows with displacement.

Our laser beams are not only red-detuned but also circularly polarized. Figure 2.15 demonstrates the interplay between the Zeeman shift of the atomic energy levels and the polarization of the light. When assembling our MOT, we want to cool the atoms by driving transitions that change the angular momentum of the atom by one unit. As shown in Figure 2.15(a), $\sigma^{+}$polarized light drives a transition with $\Delta m=+1$, while the $\sigma^{-}$beam drives a transition with $\Delta m=-1$. It is important to remember the distinction between $\sigma^{ \pm}$and right- $(R H C)$ or left-hand circular $(L H C)$ polarizations when constructing a MOT. The $\sigma$ polarizations indicate the relationship between the angular momentum $\mathbf{L}$ of 
a)

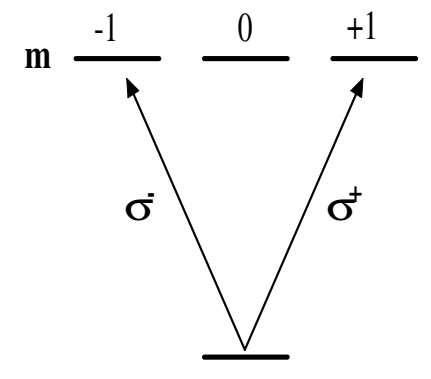

b)

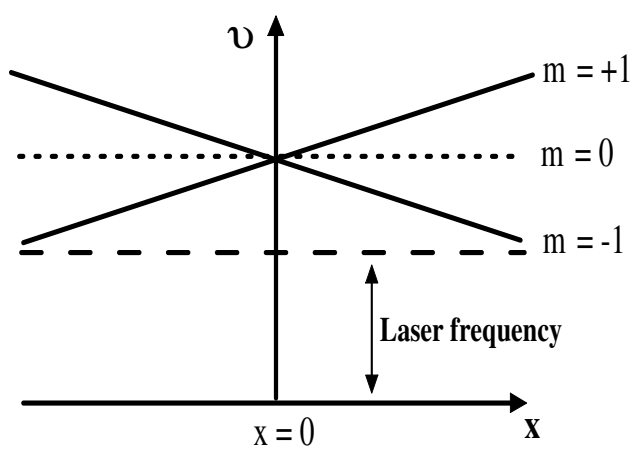

Figure 2.15: (a) shows the transitions driven in the MOT. For each counterpropagating beam pair, one beam is $\sigma^{+}$polarized and the other is $\sigma^{-}$. In part (b) the energy of each state is shown, as position varies. Due to the spatially-dependent Zeeman shift, the transitions are brought closer to resonance with laser frequency as the atoms move away from the field zero. The opposite circular polarizations also ensure that an atom will preferentially absorb from the beam directing it back towards the center of the trap, even though both beams are present everywhere in space along the beam path.

the light and the direction of the applied magnetic field,

$$
\begin{aligned}
\sigma^{-} & =\mathbf{L} 1 \downarrow \mathbf{B} \\
\sigma^{+} & =\mathbf{L} \| \mathbf{B},
\end{aligned}
$$

while $R H C$ and $L H C$ relate the angular momentum of the light to its propagation vector $\mathrm{k}$

$$
\begin{aligned}
& L H C=\mathbf{L} \| \mathbf{k} \\
& R H C=\mathbf{L} 1 \mathbf{l} \mathbf{k} .
\end{aligned}
$$

Thus depending on the direction of the magnetic field, sometimes $R H C$ is equivalent to $\sigma^{+}$and other times $\sigma^{-}$. RHC is produced by passing linearly polarized light through a quarterwave plate whose fast axis is oriented at $+45^{\circ}$ with respect to the polarization 
vector of the input beam. $L H C$ results when the fast axis is at $-45^{\circ}$.

Referring again to Figure 2.15, as we look along a beam path, we see a $\sigma^{+}$beam exiting and a $\sigma^{-}$beam entering the trapping region. As atoms diffuse through the trapping region and drift away from the center of the trap in the $+\mathbf{x}$ direction, the $\sigma^{-}$beam is brought onto resonance by the spatially-increasing Zeeman shift. At the same time the $\sigma^{+}$beam is shifted away from resonance, so that even though both beams are present everywhere along the beam path, the atoms will preferentially absorb light from the beam directing them back towards the center of the trap. If we look past the center to the other side of the trap, now the direction of the magnetic field has changed, so that the $\sigma^{+}$beam is now brought onto resonance and pushes any atoms that have strayed in that direction back towards the center of the trap.

Our experiment uses three orthogonal pairs of laser beams. Two pairs of beams are in the plane of the optics table, and the third pair is vertical. All beams have roughly equal intensity, at $6 \mathrm{~mW} / \mathrm{cm}^{2}$. The vertical beams also contain the repump light. Since the EOM converts about $10 \%$ of the incident power into the repump frequency, the total repump intensity in the vertical beams is $10 \% \times 6 \mathrm{~mW} / \mathrm{cm}^{2} \times 2=1.2 \mathrm{~mW} / \mathrm{cm}^{2}$. We find the MOT is most efficient when laser detuning is $\Delta=-20 \mathrm{MHz}$, and B field gradient is $9 \mathrm{G} / \mathrm{cm}$. With these settings we can capture atoms with approximate velocity

$$
\mathrm{v}=\nu \lambda=(20 \mathrm{MHz})(780 \mathrm{~nm}) \cong 16 \mathrm{~m} / \mathrm{s}
$$


which corresponds to a temperature of

$$
T=\frac{m v^{2}}{k_{B}}=3.5 \mathrm{~K}
$$

Thus we capture atoms already moving slowly. We use the $5^{2} S_{\frac{1}{2}} F=2 \leftrightarrow 5^{2} P_{\frac{3}{2}} F^{\prime}=3$ resonance of ${ }^{87} \mathrm{Rb}$, at $780.246 \mathrm{~nm}$, for cooling and trapping, as seen in the energy level diagram in Figure A.2.

We can see the reason for including light at the repump frequency by referring again to Eq. (2.6) to calculate the rate at which atoms spontaneously scatter photons. If we assume that our laser is $20 \mathrm{MHz}$ red of our cooling transition, and each of the six cooling beams has intensity $6 \mathrm{~mW} / \mathrm{cm}^{2}$ so that the total laser intensity is $I=36 \mathrm{~mW} / \mathrm{cm}^{2}$, we find that the scattering rate for the main cooling transition is $R_{s}=3.4 \times 10^{6}$ photons $/ \mathrm{s}$. We can also calculate the rate for off-resonant excitation to the $F^{\prime}=2$ state, $R_{s}=1.4 \times 10^{4}$ photons $/ \mathrm{s}$. If we take the ratio of the two rates, we find that for roughly every 250 photons that excite the $F=2 \leftrightarrow F^{\prime}=3$ transition there will be 1 photon that excites the $F=2 \leftrightarrow F^{\prime}=2$ transition. If we then refer to the coupling constants shown in Figure A.1, we see that an atom in the $F^{\prime}=2$ excited state has a $50 \%$ chance to decay to $F=1$, where it will no longer interact with the $780.246 \mathrm{~nm}$ radiation. If left unchecked, the process of off-resonant excitation and decay would optically pump the entire atomic population into the dark ground state within about $150 \mu \mathrm{s}$, and no further cooling would occur. Therefore we require a second laser frequency at $780.233 \mathrm{~nm}$ which excites the $F=1 \leftrightarrow F^{\prime}=2$ transition, so that atoms are repumped and can once again interact with the cooling 
beam.

In principle, laser cooling is limited only by the energy associated with an atom recoiling from emission of a photon. The recoil limit is

$$
T_{r}=\frac{\hbar^{2} k^{2}}{m k_{B}}
$$

where $\hbar k$ is the momentum of the emitted photon and $m$ is the mass of the atom. For ${ }^{87} \mathrm{Rb}$ and $780 \mathrm{~nm}$ light, the minimum temperature is $T_{r}=0.4 \mu \mathrm{K}$. The velocity associated with this temperature is $v_{r}=0.6 \mathrm{~cm} / \mathrm{s}$.

Practical MOT temperatures, however, rarely reach the recoil limit. The density of atoms in the MOT causes the cloud to become optical thick, with fewer photons penetrating to the interior of the cloud. The cooling beams lose efficiency as optical thickness increases, and the atoms do not get as cold. The density of the cloud is also limited by photon-swapping. A photon emitted by one atom may be reabsorbed by a neighboring atom. The chance of reabsorption increases with cloud density. If the cloud becomes optically thick enough, the photon can even be trapped within the cloud. Exchanging of photons in this manner is effectively a repulsive force between atoms. Because of these processes, the practical limits of our MOT are realized with $\sim 3 \times 10^{9}$ atoms at roughly $850 \mu \mathrm{K}$ with density $10^{10} \mathrm{~cm}^{-3}$. The width of the cloud is approximately $8 \mathrm{~mm}$.

We can alleviate some of the effects of optical thickness by detuning our trapping beams and/or reducing repump intensity. Detuning the lasers from resonance reduces the probability of photon absorption, giving photons time to penetrate the cloud before 
interacting with an atom. Attenuation of the repump light causes the atoms to spend more time in the $F=1$ level, which is a dark state in relation to the trapping light. Again, this reduces the probability of an atom interacting with the trapping beams, and allows a greater chance for photons to reach the interior of the cloud. While these techniques do reduce the loading rate of our trap, we can also increase cloud density. In fact, immediately after collecting atoms in our MOT for about $15 \mathrm{~s}$, we execute this procedure for $20 \mathrm{~ms}$ to produce a compressed MOT, or CMOT.

The CMOT has several advantageous features. First and foremost, it helps to increase phase space density. CMOT's are usually performed with increased detuning $\Delta$ and magnetic field gradient. When we optimized our CMOT, we found that we had the most atoms at the lowest temperatures when we increased $\Delta$ to $-38 \mathrm{MHz}$ and reduced the repump intensity by a factor of two, without changing the magnetic field gradient from the $9 \mathrm{G} / \mathrm{cm}$ used in the MOT. By reducing the optical thickness of the cloud, we obtained more efficient cooling and observed temperature of about $400 \mu \mathrm{K}$ during the CMOT. A $20 \mathrm{~ms}$ CMOT also increased the density of our cloud, thus increasing our phase space density.

The CMOT also helps optimize the MOT stage, because it reveals the center of the trap. This can be difficult to discern during the full MOT because imbalances of intensity or polarization between counterpropagating beam pairs can push the cloud to one side. We adjust the beam balance so that the MOT and CMOT are concentric. The procedure we followed was to write a sequence of operations to be carried out by our ADwin pulsing system, which would run the MOT for one second and then switch to CMOT conditions 
for a few ms. We monitored the cloud with an infrared camera and displayed the feed on a television monitor, so we could watch the position of the MOT in comparison to the CMOT as we adjusted beam balances. I mention this experimental note in order to point out an important aspect of AOM's - they tend to exhibit temperature sensitivity. An AOM that is on for long periods of time heats up, and the angle of its deflected output beam may change. When using this pulsing sequence, it was important to turn on the CMOT for only a short fraction of the time that the MOT was on. This was due to the fact that we were changing $\Delta$ by applying a voltage to the double-pass AOM, which heated up rapidly. By not paying attention to this effect, we at times aligned the MOT and CMOT improperly, and spent a lot of time trying to find a problem that didn't exist. For any beam that is pulsed quickly by means of an AOM, beam alignment should be performed during a similar pulsing process.

Beam imbalance can also be diagnosed by suddenly releasing the cloud from the MOT by turning off the magnetic field, and watching it expand. Slow, uniform expansion indicates properly balanced laser beam intensity and zero background field. Nonuniform expansion indicates the MOT is not properly aligned and/or balanced, or that a stray magnetic field is present. Correction of this error often results in improved MOT efficiency.

We would like to laser cool the cloud as much as we can before moving on to another cooling technique. To that end, we employ an optical molasses immediately after the CMOT. The optical molasses works through another laser cooling technique known as polarization gradient cooling. When the MOT was first developed, investigators were surprised to find lower temperatures than they had expected. Polarization gradient cooling 
was eventually determined to be the cause. A discussion of $\sigma^{+}-\sigma^{-}$polarization gradient cooling is beyond the scope of this chapter, but can be found in [42]. We use all six MOT beams to perform the optical molasses, with the magnetic field switched off, and observe a cloud temperature of $200 \mu \mathrm{K}$.

Laser cooling techniques such as the MOT, CMOT, and optical molasses bring our Rb atoms significantly closer to condensation by collecting $3 \times 10^{9}$ atoms at $\sim 200 \mu \mathrm{K}$ with

density of a few times $10^{10} \mathrm{~cm}^{-3}$. However, the density must still be increased to more than $10^{12} \mathrm{~cm}^{-3}$ and the temperature lowered to a few hundred $\mathrm{nK}$. Therefore another cooling technique is required. Since the MOT is used to cool and collect atoms at one location, we will also need to find another way to hold on to the atoms while we continue cooling. For this purpose we employ a magnetic trap.

\subsection{Magnetic Trap}

Magnetic trapping of neutral atoms was first observed in 1985 [43]. An atom is confined through the interaction between the field and the magnetic moment of the atom, resulting in a potential

$$
U=-\vec{\mu} \cdot \mathbf{B} .
$$

The force on the atom is then

$$
\mathbf{F}=\nabla(\vec{\mu} \cdot \mathbf{B}) .
$$

For a given Zeeman level, the angle between $\vec{\mu}$ and $\mathbf{B}$ is fixed, so that $\mathbf{F}=\mu \nabla|B|$ where $\mu$ is the projection of the magnetic moment along $\mathbf{B}$. Through this force, kinetic energy 
is translated into potential energy, and the atom is confined. As long as the motion of the atom in the trap is adiabatic, the angle between $\vec{\mu}$ and $\mathbf{B}$ is preserved so that the atom remains in the same $m$ level, and can be trapped indefinitely. According to our force equation confinement occurs at a local maximum of the B field for atoms with a negative magnetic moment $\mu$, and at a local $\mathrm{B}$ field minimum for atoms with positive magnetic moment. Since local B field maxima are not allowed in a region with zero electrical currents, our atoms must have a positive magnetic moment in order to be trapped [70].

The magnetic moment can be expressed as

$$
\mu=g_{F} m_{F} \mu_{B}
$$

where $\mu_{B}$ is the Bohr magneton, $m_{F}$ is the magnetic quantum number, and $g_{F}$ is the Landé $g$ factor of the hyperfine level. Each energy level has its own characteristic $g_{F}$, given by

$$
g_{F} \approx g_{J} \frac{F(F+1)+J(J+1)-I(I+1)}{2 F(F+1)}
$$

where $g_{J}$ is the fine structure Landé $g$-factor, $I$ is the nuclear spin of the atom, $F$ is the hyperfine level and $J$ is the sum of the orbital and electron spins. For the $5^{2} S_{1 / 2}$ state of ${ }^{87} \mathrm{Rb}, I=3 / 2, J=1 / 2$, and as shown in Table A.3 $g_{J}=2.0$. Thus for $F=2$ we find that $g_{F}=1 / 2$, while for $F=1$ we obtain $g_{F}=-1 / 2$.

Within each hyperfine level, only certain spin states will result in a positive magnetic moment. Applying the condition of $\mu>0$, for the $F=1$ ground state only the $|1-1\rangle$ state is trapped. In the $F=2$ level there are two trapped states, $|21\rangle$ and $|22\rangle$. Their 
magnetic moments are

$$
\begin{gathered}
\mu_{|1,-1\rangle}=\mu_{B} / 2 \\
\mu_{|2,1\rangle}=\mu_{B} / 2 \\
\mu_{|2,2\rangle}=\mu_{B} .
\end{gathered}
$$

The most convenient trap configuration is a quadrupole field, just as in the MOT. For the field generated by two stacked current-loops,

$$
\mathbf{B}=B_{z}^{\prime}\left(z \mathbf{z}-\frac{x}{2} \mathbf{x}-\frac{y}{2} \mathbf{y}\right)
$$

the potential has the form

$$
V=\mu|\mathbf{B}|=\mu B_{z}^{\prime} \sqrt{z^{2}+\frac{x^{2}+y^{2}}{4}}
$$

where $\mathbf{z}$ is along the common axis of the coils. The potential is $\mathrm{V}$-shaped, growing in magnitude with distance from the field zero. Atoms with $\mu>0$ can be confined by this potential.

The MOT uses a field gradient of $B_{z}^{\prime}=9 \mathrm{G} / \mathrm{cm}$, which is too weak for a pure magnetic trap. If we set the force in Equation 2.23 equal to $\mathrm{mg}$, we find that a $15 \mathrm{G} / \mathrm{cm}$ gradient is required to support atoms in the $|22\rangle$ state against gravity, and $30 \mathrm{G} / \mathrm{cm}$ for $|21\rangle$ and $|1-1\rangle$. At full strength, our magnetic trap produces a gradient of $387 \mathrm{G} / \mathrm{cm}$, creating a potential that is approximately $50 \mathrm{mK}$ deep, as measured to the chamber walls. 
Our MOT contains atoms in many different spin states. If we turn off the trapping light, the atoms would distribute themselves roughly equally among the five spin states in the $F=2$ ground state hyperfine level. Since only two of these states have $\mu>0$, only $40 \%$ of the atoms could be trapped in this case. However the two spin states involved have different magnetic moments and so the atoms would experience a different potential depending on which state they are in. If we were to leave the trapping light on and turn off the repump light, then the atoms would be divided among the three spin states in the $F=1$ level. Now we could potentially trap $33 \%$ of the population in one state. In any case, simply capturing atoms directly from the MOT will not transfer much of the population into the magnetic trap.

We initially attempted to use the $|1,-1\rangle$ state for our BEC experiment, by merely turning off the repump light and allowing the atoms to settle into the $F=1$ manifold as described above. We were not able to achieve condensation, however, because our elastic collision rate was never large enough to establish run-away evaporation. Our efforts in this regard are discussed further in Chapter 3.

For higher transfer efficiency, we instead optically pumped the atoms into the $|22\rangle$ state. Atoms in the this state have twice the magnetic moment and experience twice the trapping potential of atoms in either of the other two trapped states. The tighter trap causes the collision rate to increase. This state also experiences a higher rate of threebody losses, but the benefit of the higher collision rate seems to more than compensate for the higher loss rate in our case.

We optically pump the atoms into the $|2,2\rangle$ state by turning off the trapping and 
repump beams for the MOT and the magnetic field gradient. We then turn on another beam tuned to the $F=2 \leftrightarrow F^{\prime}=2$ transition, which is $247 \mathrm{MHz}$ red of the cooling and trapping beams. We used the undeflected beam from the repump AOM to create the optical pumping (OP) beam. The OP beam passes through another Crystal Tech AOM twice, with frequency shift $\Delta \nu=-84 \mathrm{MHz}$ per pass for a total shift of $-168 \mathrm{MHz}$. Since this beam does not receive $+80 \mathrm{MHz}$ from the repump AOM, the total detuning of the beam is $-168-80=-248 \mathrm{MHz}$ from the main trapping light. The intensity of the OP beam is $300 \mu \mathrm{W} / \mathrm{cm}^{2}$ with diameter $2 \mathrm{~cm}$, so that the light encompasses the entire atomic cloud. The beam passes through the MOT chamber to a retroreflection mirror, which sends the beam back upon itself and through the atoms a second time.

The OP beam is $\sigma^{+}$polarized, stepping the atoms from state to state through the $F=2$ level until they come to $m_{F}=2$. Since the $F^{\prime}=2$ excited state contains no $m_{F}^{\prime}=3$ level, there are no further excitations from the optical pumper beam, making $m_{F}=2$ a dark state. We turn on a $2 \mathrm{G}$ bias field along the path of the OP beam to promote pumping efficiency. After $1 \mathrm{~ms}$ of optical pumping we observe $80 \%$ of the MOT atoms pumped into the dark state. While the MOT beams and magnetic field are off, we must be careful not to let the cloud expand out of the trap. Since the atoms are moving at $14 \mathrm{~cm} / \mathrm{s}(T \sim 200 \mu \mathrm{K})$, they move only $1.5 \mathrm{~mm}$ during the optical pumping stage, and the cloud does not significantly expand.

During the optical pumping phase we still need repump light. While our OP beam has indeed passed through the EOM, the repump light has also been detuned by $-248 \mathrm{MHz}$ so that the scattering rate is small. Instead, we tune the Toptica diode laser to the 

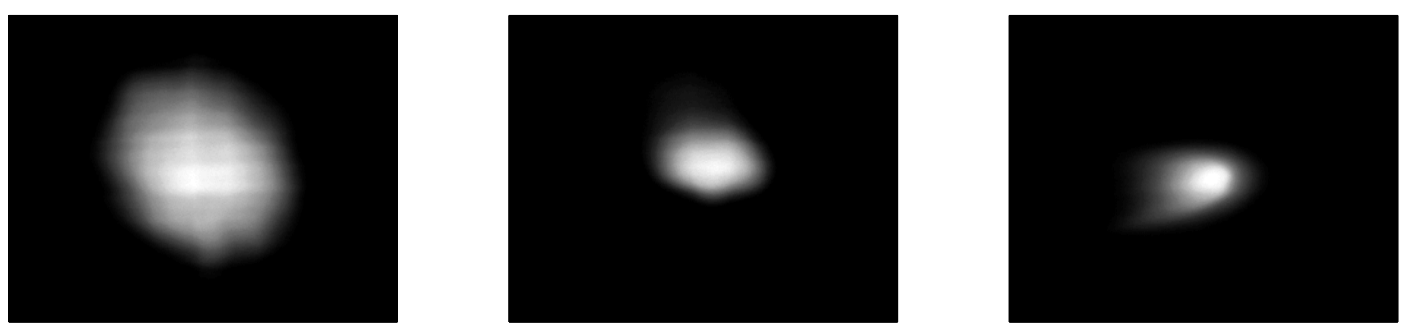

Figure 2.16: Fluorescence images of trapped atom cloud. The first image is the MOT, where the atoms are captured from the background vapor and cooled. Our MOT is fairly large, containing about $3 \times 10^{9}$ atoms. However it is also almost opaque to the cooling beams, so that the average temperature of the cloud is about $800 \mu \mathrm{K}$. The second image is the CMOT. As the picture shows, the cloud is significantly compressed here, but we maintain essentially the entire MOT population. Also the efficiency of the cooling beams is improved, so that the temperature reaches $400 \mu \mathrm{K}$. Finally, the magnetic trap contains about $2 \times 10^{9}$ atoms at $900 \mu \mathrm{K}$. The width of the CMOT is chosen to roughly match the width of the magnetically trapped cloud.

$F=1 \leftrightarrow F^{\prime}=2$ transition. This beam is then directed into the atomic cloud, providing $0.75 \mathrm{~mW} / \mathrm{cm}^{2}$ of pure repump light. Ideally, the repump and OP beams would be colinear along the direction of the bias field, so that all of the OP light would drive $\sigma^{+}$transitions. However, achieving this condition would require sacrificing some of our laser power, and so we chose instead to tolerate a small angle $\left(\sim 30^{\circ}\right)$ between the beams.

After optical pumping the lasers are turned off entirely and the magnetic field is turned on at $124 \mathrm{G} / \mathrm{cm}$ for $50 \mathrm{~ms}$ so that the atoms are caught in the magnetic trap but not yet tightly compressed. Over $200 \mathrm{~ms}$ the field is adiabatically ramped up to our maximum possible gradient of $387 \mathrm{G} / \mathrm{cm}$. Ballistic flight analysis, described in Chapter 3, reveals that the cloud temperature has risen to $900 \mu \mathrm{K}$.

The temperature of the cloud in the magnetic trap is largely dependent upon the CMOT stage. In a healthy CMOT, the lasers are balanced and stray fields are shielded or compensated for, keeping the CMOT temperature below $300 \mu \mathrm{K}$. The location of the 
cloud is also at or near the field zero, which is the center of the magnetic trap. If the MOT and CMOT are not well-centered, the cloud will "slosh" into the magnetic trap and the atoms will heat up. The reduced cloud size of the CMOT also helps to minimize the potential energy gained when the atoms are transferred to the much tighter potential of the magnetic trap. Our CMOT compresses the cloud to a Gaussian width of about $2.5 \mathrm{~mm}$, which matches the width of the cloud in the magnetic trap. Figure 2.16 shows the relative sizes of the MOT, CMOT and magnetic trap. 


\section{Chapter 3}

\section{Bose-Einstein Condensation}

The phase space density of our magnetically trapped atoms is $\rho=5 \times 10^{-8}$. We must continue cooling the cloud until $\rho \cong 2$ in order to make a condensate. In this chapter, I discuss how we used evaporative cooling to produce the first Bose-Einstein condensates at the University of Virginia. I will first explain the design and construction of the magnetic trap in which evaporation was performed. The entire trap is carried to the science cell by a specialized translation system. I will provide the step-by-step procedure used for evaporation, and present images of our condensates. Finally I will discuss our imaging system and how we used it to analyze the condition of the cloud.

\subsection{Construction of Trap For Evaporative Cooling}

In the previous chapter, I described the general operation of our magnetic trap. There are several features of the trap especially designed to enable evaporative cooling. While the principles of evaporation are explained in the next section, one requirement is that 
the atomic cloud must have a high elastic collision rate,

$$
R_{c}=n \sigma \bar{v}
$$

where $n$ is the density of the cloud, $\sigma$ is the scattering cross section and $\bar{v}$ is the average relative velocity of the atoms. Since our cloud is cold, we increase the collision rate by using a tight trap that compresses the cloud to high density. Our magnetic trap must be capable of supplying a field gradient $B_{z}^{\prime} \sim 400 \mathrm{G} / \mathrm{cm}$.

The magnetic field in our trap is generated by two anti-Helmholtz coils, as shown in the last chapter. Our coils are constructed out of $1 / 4$ inch-outer-diameter copper tubing, wound into 16 turns so that the outer diameter of the finished coil is $122 \mathrm{~cm}$ and the inner diameter is $45 \mathrm{~cm}$. The tubing is insulated with fiberglass electrical tape. Each coil is epoxied to a Delrin coilholder. Appendix E contains a mechanical sketch of the coilholders. The coilholders are attached with steel screws to an aluminum tower $178 \mathrm{~cm}$ tall and $51 \mathrm{~cm}$ wide, which suspends the coils above and below the MOT chamber. The coils are vertically separated by $64 \mathrm{~cm}$, which is the diameter of the MOT chamber. The entire assembly is illustrated in Figure 3.1.

We generate the current through the coils with our "B field control panel". The design of this panel was the subject of Ben Deissler's Master's thesis [22], which should be referenced for a detailed explanation. The B field controller accepts an analog input control voltage $V_{c}$ from our ADwin pulsing system, and supplies $75 \mathrm{~A}$ of current through the B field coils per $1 \mathrm{~V}$ input. At $V_{c}=10 \mathrm{~V}$, the controller reaches its maximum current 


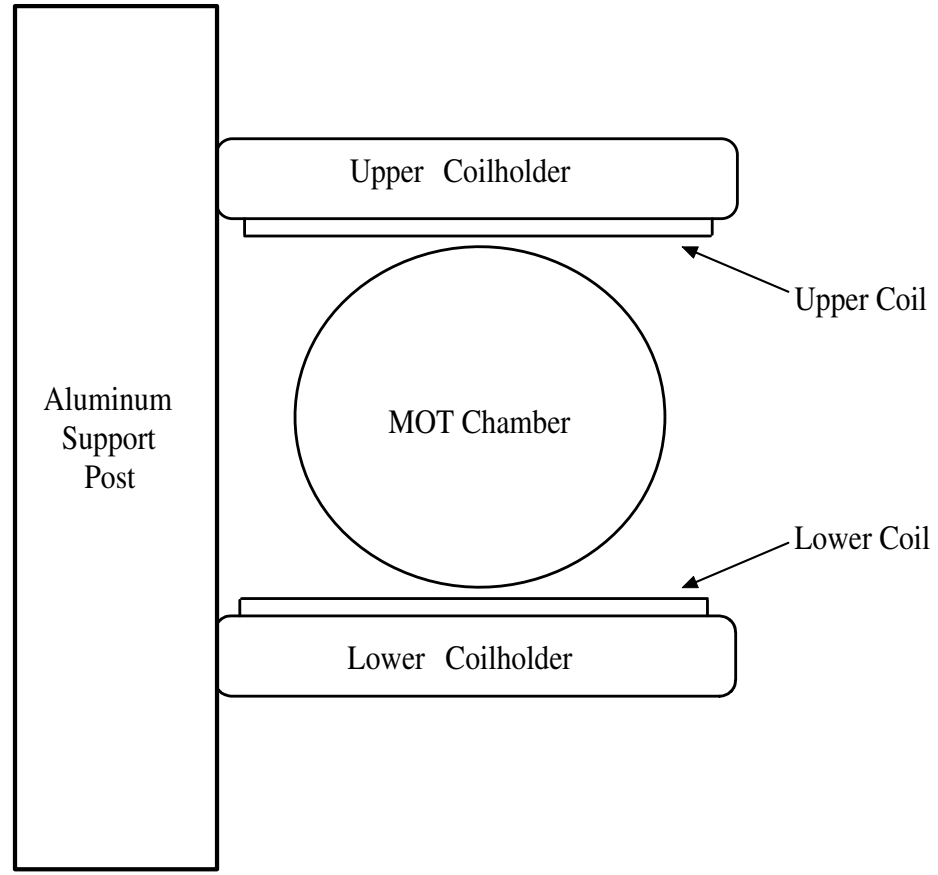

Figure 3.1: Sketch of magnetic trap coilholder system. The MOT chamber vacuum cell is viewed from one end, so that its cross-section is a circle. Each coil is secured to a Delrin coilholder with epoxy. The coilholders are attached with steel screws to an aluminum tower that suspends the coils above and below the MOT chamber. We place the coils as close to the chamber as possible without touching it, in order to maximize the field gradient at the atoms.

output of $750 \mathrm{~A}$. The field gradient generated is about $1 \mathrm{G} / \mathrm{cm}$ per $2 \mathrm{~A}$ current through the coils. The maximum measured field gradient is $B_{z}^{\prime}=387 \mathrm{G} / \mathrm{cm}$.

The total resistance of the B field coils is about $17 \mathrm{~m} \Omega$, and heat dissipation is a significant concern. We run chilled water through the coils at all times to prevent overheating and possible melting of the coil assembly. The cooling water is $20^{\circ} \mathrm{C}$ and flows at a rate of $2.5 \mathrm{~L} / \mathrm{min}$. The water flows through the coils in parallel, so that the flow rate per coil is $1.25 \mathrm{~L} / \mathrm{min}$. Due to equipment malfunction and user error, we experienced three catastrophic coil-explosion incidents. The fundamental problem was reduction or stoppage of the flow of chilled water. The temperature of the coils exceeded $100^{\circ} \mathrm{C}$, causing the water to boil within the tubing. The extreme stress on the coils resulted in leaks 
at weak points like solder joints and plastic patch tubing, spraying water over the optics table and vacuum chamber. To help prevent these incidents, we installed two interlock systems that when tripped interrupted the output of the B field control panel, shutting off the current through the coils. Interlock 1 was a flow meter with an alarm, that monitored the flow of chilled water through the system. We placed the flow meter on the output of our entire chilled water delivery manifold. We then set the flow meter alarm to switch on when the flow rate fell just below the normal level. When the flow meter alarm tripped, it opened a switch within the B field controller, shutting off the current through the coils. Interlock 2 directly monitored the temperature of the coils, with a set point of $60^{\circ} \mathrm{C}$ to trip and open the same switch. This interlock was wired in series with Interlock 1, so that if either circuit was tripped the current through the coils was immediately shut off. Since these interlocks were added, we have not experienced any further overheating incidents.

The B field coils generate a magnetic field

$$
\mathbf{B}=B_{z}^{\prime}\left(z \mathbf{z}-\frac{x}{2} \mathbf{x}-\frac{y}{2} \mathbf{y}\right)
$$

The potential associated with this field is

$$
V=\mu|\mathbf{B}|=\mu B_{z}^{\prime} \sqrt{z^{2}+\frac{x^{2}+y^{2}}{4}} .
$$

Given the general form for the density distribution $n(\mathbf{r})=n_{0} e^{-V(\mathbf{r}) / k_{B} T}$, we can find the 
number of atoms in the trap

$$
N=\int n(\mathbf{r}) d^{3} r=32 \pi n_{0} r_{1}^{3}
$$

where $n_{0}$ is the peak density and $r_{1}=k T / \mu B^{\prime}$ is the radius of the cloud. Alternatively, we can solve for the peak density

$$
n_{0}=\frac{N}{32 \pi r_{1}^{3}} .
$$

Finally, the average density is

$$
\bar{n}=\frac{1}{N} \int n^{2}(\mathbf{r}) d^{3} r=\frac{N}{256 \pi r_{1}^{3}}=\frac{1}{8} n_{0} .
$$

At maximum operating strength of $B_{z}^{\prime}=387 \mathrm{G} / \mathrm{cm}$, the depth of our trap is

$$
\frac{\mu B^{\prime} z}{k_{B}}=50 \mathrm{mK}
$$

at a distance $z=2 \mathrm{~cm}$ from the center of the coils. We load our magnetic trap directly from the MOT, where the background vapor pressure is typically $P_{\text {мот }}=7 \times 10^{-10}$ torr when the $\mathrm{Rb}$ getters are running. The pressure is $P_{\text {мот }}=3 \times 10^{-10}$ torr when the getters are off. Because of its proximity to the Rb getters, the MOT is surrounded by a hot Rb vapor out of which the coldest atoms are cooled and trapped. MOT atoms are thus constantly bombarded by high-energy atoms, and knocked out of the MOT. Losses due to collisions with background atoms do not significantly limit the population of our MOT because the laser beams continuously replenish our stock of cold atoms. However, once the lasers 
are turned off and the atoms are magnetically trapped, we can no longer replace atoms lost to background collisions. Furthermore the temperature of the background vapor is approximately $300 \mathrm{~K}$. Accordingly, even a glancing collision with a highly energetic atom from the background vapor could easily dislodge an atom from our trap.

We suppress collisional losses from the magnetic trap by transporting it to a second vacuum chamber, where the pressure is $P_{\text {science }}=4 \times 10^{-11}$ torr. In this ultra-high vacuum the lifetime of the atoms in the trap is $80 \mathrm{~s}$, compared to 3 or $4 \mathrm{~s}$ in the MOT chamber. The two chambers are isolated from each other by a glass tube $30 \mathrm{~cm}$ long and $1 \mathrm{~cm}$ in diameter. Noting that the average energy of the atoms in the trap is $\frac{9}{2} k_{B} T$, we make the diameter of the cloud well under $1 \mathrm{~cm}$ or much of the population will be lost to collisions with the walls of the tube during the transfer.

Adiabatic compression of the cloud is useful not only for optimizing transfer through the tube, but also for understanding how to load the magnetic trap efficiently and maximizing the collision rate for evaporative cooling. For an adiabatic compression, the temperature and field gradient are related according to

$$
T \propto\left(B^{\prime}\right)^{2 / 3},
$$

so that the radius of the cloud goes as

$$
r \propto \frac{T}{B^{\prime}}=\left(B^{\prime}\right)^{-1 / 3} .
$$

We previously defined the radius of the cloud so that $B^{\prime}=k T / \mu r$. If we catch the 
atoms at gradient $B_{i}^{\prime}$ and then compress the cloud with maximum gradient $B_{f}^{\prime}$, the final temperature will be

$$
T_{f}=T_{i}\left(\frac{B_{f}^{\prime}}{B_{i}^{\prime}}\right)^{2 / 3}
$$

with final radius

$$
r_{f}=r_{i}\left(\frac{B_{i}^{\prime}}{B_{f}^{\prime}}\right)^{1 / 3}
$$

Substituting for $B_{i}^{\prime}$,

$$
\begin{aligned}
& T_{f}=T_{i}\left(B_{f}^{\prime} \frac{\mu r_{i}}{k T_{i}}\right)^{2 / 3} \sim B_{f}^{\prime 2 / 3} r_{i}^{2 / 3} T_{i}^{1 / 3} \\
& r_{f}=r_{i}\left(\frac{k T_{i}}{\mu r_{i}} \frac{1}{B_{f}^{\prime}}\right)^{1 / 3} \sim B_{f}^{\prime-1 / 3} r_{i}^{2 / 3} T_{i}^{1 / 3}
\end{aligned}
$$

We see the dependence of cloud size on field gradient is weak, so that increasing $B^{\prime}$ does not excessively compress the cloud. We consistently observed $75 \%$ of the atoms successfully transferred through the tube to the science cell for gradients as low as $200 \mathrm{G} / \mathrm{cm}$. We maximized the collision rate prior to evaporation by running the magnetic trap at maximum gradient, but in order to load the trap efficiently we used this adiabatic compression technique to execute a "catch and compress" loading procedure. The atoms were initially caught in the magnetic trap with field gradient $124 \mathrm{G} / \mathrm{cm}$, and then the cloud was adiabatically compressed by ramping the field up to $387 \mathrm{G} / \mathrm{cm}$. In this way we loaded up to $80 \%$ of the MOT atoms into the magnetic trap, and minimized the rise in temperature as we tightened the trap in preparation for evaporative cooling.

Once the cloud has been adiabatically compressed, the atoms are transported to the 
UHV chamber, which we call the science cell, by a translating magnetic trap. Modeled after the system described in [36], the translating trap efficiently transfers the atomic population of the magnetic trap to the science cell. The translation system consists of a motor that drives a ball screw, moving a mounting carriage along a track lying parallel to the vacuum chamber. Our system uses a model BE231DJ-NMSN brushless servo motor from Parker Compumotor, that drives the mounting carriage down a $600 \mathrm{~mm}$ track. The carriage moves $10 \mathrm{~mm}$ with every revolution of the ball screw. The motor is capable of applying $0.58 \mathrm{Nm}$ of torque when the screw rotates at $5000 \mathrm{rpm}$ [4]. We assembled the translation track next to our vacuum chamber, running from MOT chamber to science cell. We secured our coilholder assembly to the mounting carriage with an aluminum mounting plate. Figure 3.2 illustrates this assembly. Figure 3.3 shows the translation track in relation to the vacuum chamber. The mechanical drawings for the coilholders, aluminum tower, and aluminum mounting plate are included in Appendix E.

We move the atoms a total distance of $543 \mathrm{~mm}$ in $1.758 \mathrm{~s}$, into the science cell. The motion has three distinct stages. The atoms are first accelerated at $100 \mathrm{~cm} / \mathrm{s}^{2}$ for $400 \mathrm{~ms}$, which moves them $80 \mathrm{~mm}$ and approaches the exit of the MOT cell. This brings the atoms to a cruising velocity of $40 \mathrm{~cm} / \mathrm{s}$, so that they travel $383 \mathrm{~mm}$ over the next $958 \mathrm{~ms}$. During the final stage the atoms are decelerated at $-100 \mathrm{~cm} / \mathrm{s}^{2}$ for $400 \mathrm{~ms}$ over the last $80 \mathrm{~mm}$ of travel. Figure 3.4 shows the position, velocity, and acceleration of the atoms throughout the motion. The Compumotor software package "MotionPlanner" allows the system to be programmed to execute the motions described in sequence. It also accepts a trigger input. We trigger initiation of the motion $200 \mathrm{~ms}$ after the magnetic trap is 


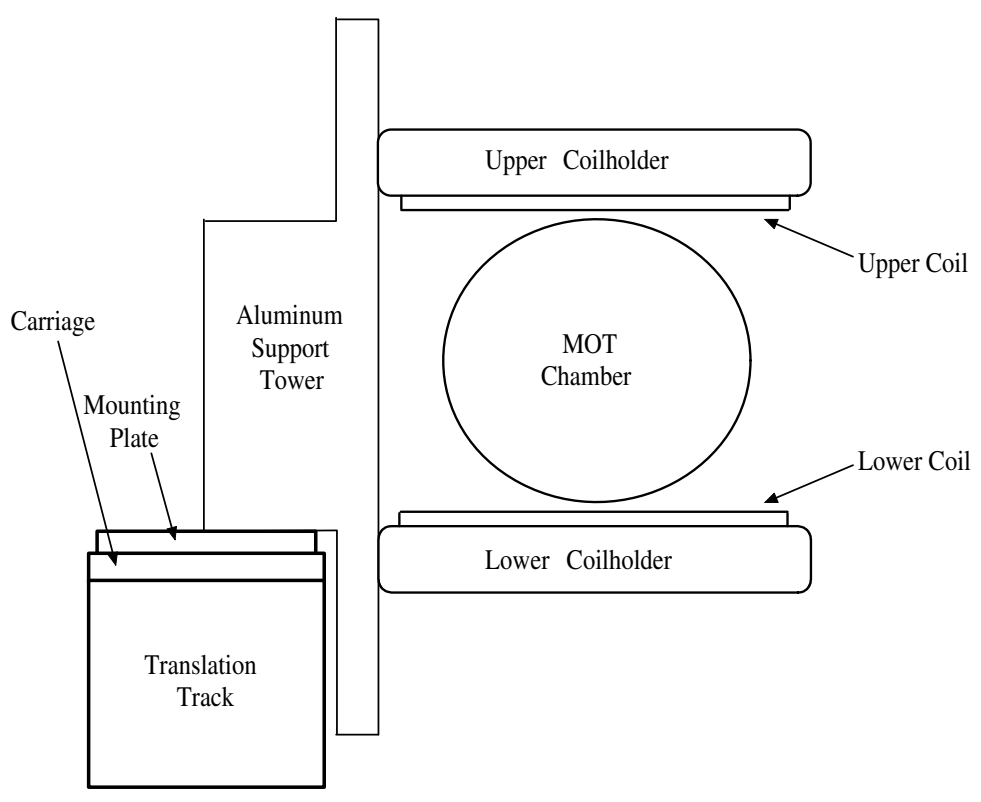

Figure 3.2: The electromagnet coils are epoxied into Delrin coilholders, which are attached with steel screws to an aluminum support tower. The tower is attached with steel screws to an aluminum mounting plate, which is attached with screws to the carriage. This view is from the science cell end of the vacuum chamber.

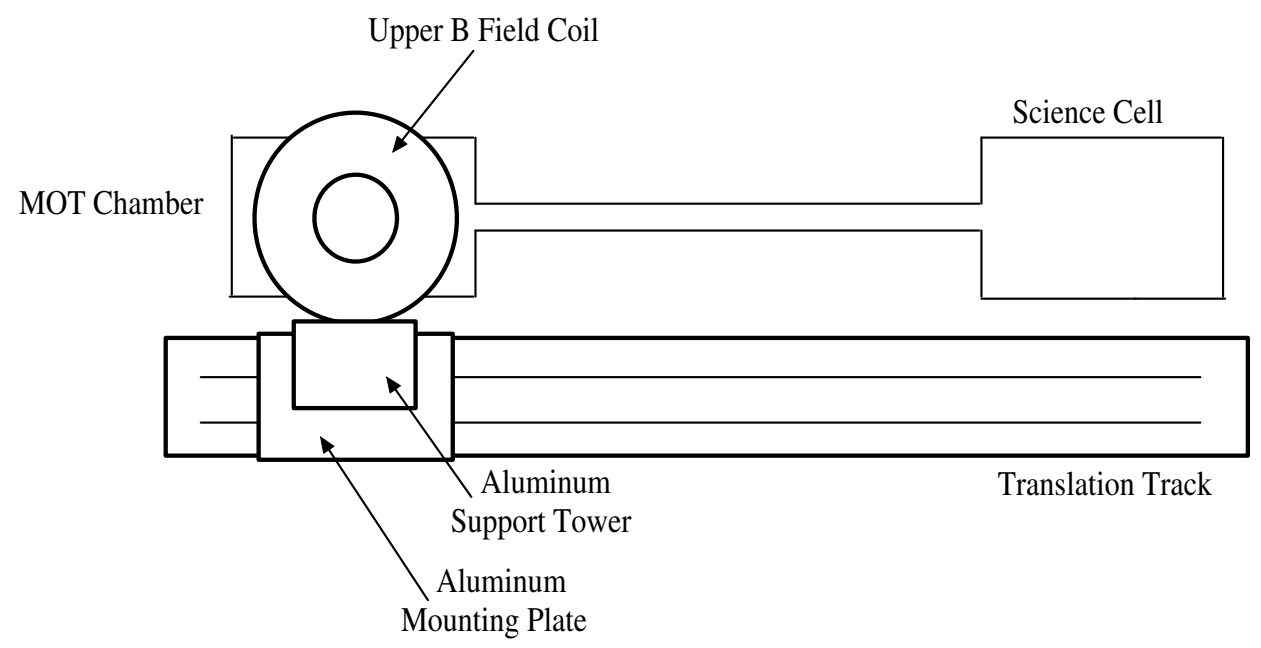

Figure 3.3: The translation track is aligned along the axis of the vacuum chamber. This view is from above the chamber, showing the B field coils mounted to the track and the path of the atoms from the MOT chamber to the science cell. Since this is a top view, the lower B field coil is directly beneath the upper coil, and the translation carriage is directly beneath the aluminum mounting plate. 

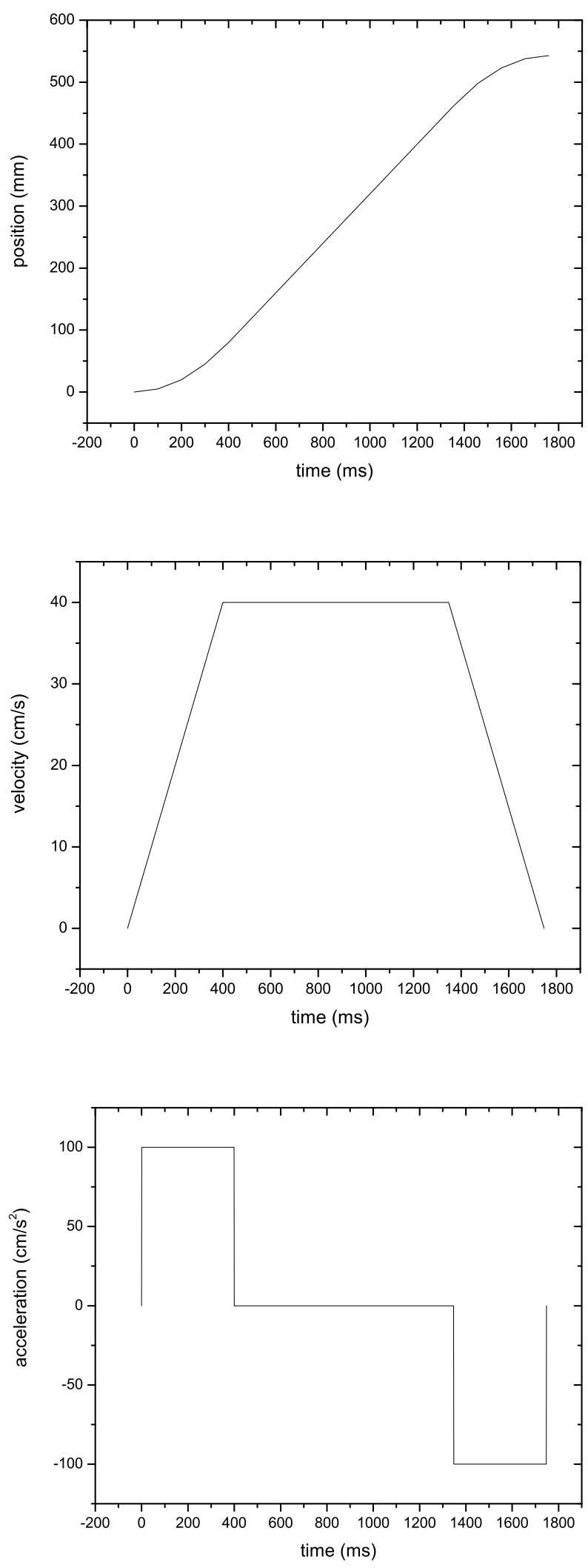

Figure 3.4: Movement of magnetic trap to science cell. Our compumotor system transports the atoms with maximum velocity of $40 \mathrm{~cm} / \mathrm{s}$, taking $1.758 \mathrm{~s}$ to move $543 \mathrm{~mm}$ to the science cell. 
loaded.

The mass of our magnetic coils, control cables, and mounting assembly is approximately $15 \mathrm{~kg}$, and so to execute this motion we require a peak force of $15 \mathrm{~N}$ from the motor. Given that our carriage moves $10 \mathrm{~mm}$ per revolution of the lead screw, the peak torque is then

$$
T=F \frac{d}{\theta}=15 \mathrm{~N} \frac{10 \mathrm{~mm}}{2 \pi}=0.024 \mathrm{Nm} .
$$

While this is well below the motor's rated torque of $0.58 \mathrm{Nm}$, we found that faster movement causes the temperature of the cloud to increase, probably due to vibrations of the trap. The placement of the carriage has specified precision $\pm 5 \mu \mathrm{m}$.

\subsection{Evaporative Cooling Principles}

Once the atoms are transferred to the science cell, we can begin the next cooling process. We use evaporative cooling to preferentially remove atoms with higher than average energy. The atoms that remain rethermalize through elastic collisions, and the average temperature of the cloud is reduced. Evaporative cooling was first applied to trapped atoms in 1988 [41]. One common example of evaporative cooling is to consider a hot cup of coffee. The most energetic molecules in the coffee break away from the surface of the liquid and escape as steam. Over time, many of the hot atoms escape, which lowers the average energy of the atoms remaining, and the coffee cools. Our trapped atoms do not have enough energy to escape over the lip of the trap. Instead, we create an artificial lip that allows atoms with higher energy to escape. 
We know that in the magnetic trap, the Zeeman effect is spatially-dependent. The further an atom travels from the center of trap, the larger the Zeeman shift of its energy levels. As first proposed in 1986 [31], we can apply radio-frequency (RF) radiation to drive transitions between atomic spin states. The RF field will only be in resonance with the spin transitions at a specific value of the B field,

$$
B=\frac{h \nu}{g_{F} \mu_{B}},
$$

where $\nu$ is the frequency of the RF photon, $g_{F}$ is the Landé $\mathrm{g}$ factor and $\mu_{B}$ is the Bohr magneton. We usually select the energy of the RF field to be about 6 times $k_{B} T$ of the atoms, so that only atoms with greater than average energy will make the spin transitions. Recalling that we must have $\mu>0$ in order for an atom to be trapped, these spin transitions will flip the sign of $\mu$ so that the hot atoms switch from weak-fieldseeking to strong-field-seeking, and are ejected from the trap. Since only the lower energy atoms remain, they collide and rethermalize at a lower average temperature than before. Figure 3.5 shows a sketch of the energies of the RF photons and atomic spin states.

To continuously cool the cloud, we scan the RF radiation to lower frequencies. This effectively reduces the depth of the trap, allowing more atoms to leave. The rate of the frequency scan is important, however, since we must allow the atoms time to rethermalize during the evaporation process. Our rate of scan was determined empirically.

Although we discard atoms during evaporation, these atoms carry a disproportionate share of the total energy of the cloud. Therefore by removing a relatively small number of 


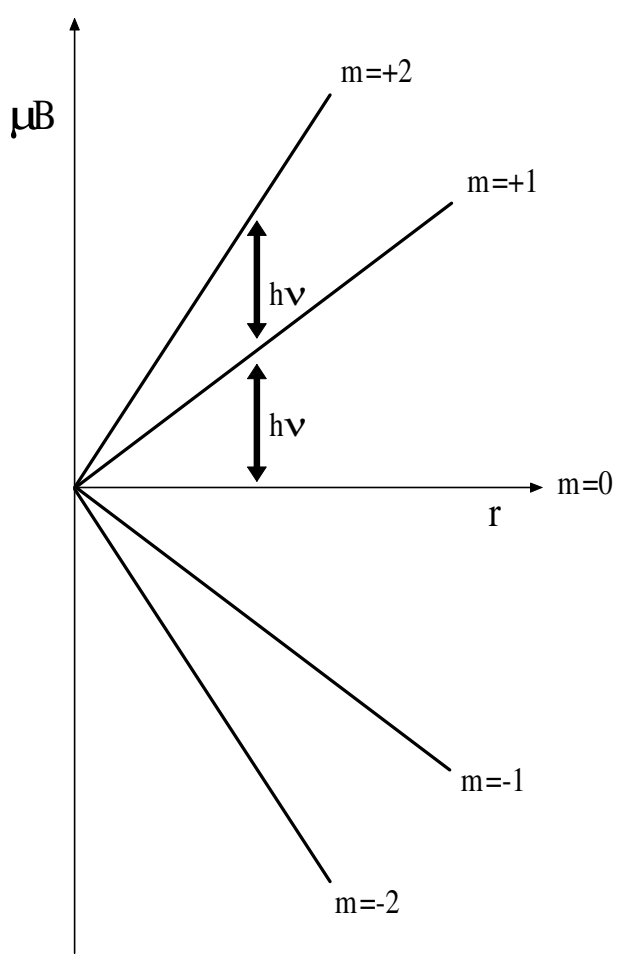

Figure 3.5: Energies of RF field and atomic spin states during evaporative cooling. The RF energy $h \nu$ is resonant with a transition between Zeeman levels only at the appropriate distance $r$ from the center of the trap. Atoms with enough kinetic energy to travel that distance away from the trap will make RF transitions to anti-trapped spin states and leave the trap.

atoms, we can cool the cloud significantly. The cooled atoms also spend more time near the bottom of the trap, so that evaporative cooling works to increase the density of the cloud as well as lower the average temperature. As a result, the phase space density of the atoms is increased.

One important loss mechanism is collisions with the background vapor. Although we perform evaporative cooling in the UHV science cell, the background pressure still limits the lifetime of the cloud to about $80 \mathrm{~s}$. It is to our advantage to evaporate as quickly and efficiently as possible so as to reduce the impact of the background vapor. Consequently, high collision rates are desirable. However higher collision rates also result in increased 
losses due to three-body collisions. The total loss rate is

$$
R_{\mathrm{T}}=R_{\text {lifetime }}+R_{\text {3body }},
$$

where $R_{\text {lifetime }} \sim 1 / 80 \mathrm{~s}$ and $R_{3 \mathrm{body}}=G_{3} n^{2}$. The measured value of $G_{3}$ is $1.8 \times 10^{-29} \mathrm{~cm}^{6} / \mathrm{s}$ [62]. As the atoms get colder, we must keep the density in an optimum range so that the losses do not overwhelm the evaporation process. In general, we try to keep $R_{\mathrm{T}} \leq R_{c} / 150$. The other loss mechanism we must address is spin disorientation. While the angle between $\vec{\mu}$ and $\mathbf{B}$ is normally fixed due to the adiabatic motion of the atoms in the trap, that angle can change when an atom passes through the field zero. The momentary loss of spin alignment allows the atom to reorient itself, thereby transitioning to a different spin state which may not have $\mu \geq 0$. These spin disorientations are known as "Majorana flips" [39]. Since our cloud is trapped about a field zero, we suffer Majorana losses at the center of the trap. The lifetime of the cloud due to Majorana losses is given by

$$
\tau=\frac{1}{4} \alpha \sigma_{F W H M}^{2},
$$

where $\alpha=3.7 \times 10^{4} \mathrm{~s} / \mathrm{cm}^{2}$ is experimentally determined [54] and $\sigma_{F W H M}$ is the full width half max of the cloud. We can convert to the measured width $w$ of a Gaussian fit to the density of the cloud so that the lifetime is

$$
\tau=\alpha \ln 2 \frac{w^{2}}{1.8^{2}}=w^{2} \times 80 \mathrm{~s} / \mathrm{mm}^{2} .
$$


At $900 \mu \mathrm{K}$ prior to the beginning of evaporative cooling, our atoms are still highly energetic and spend most of the time away from the center of the trap. When the cloud is cooled to about $200 \mu \mathrm{K}$, Majorana flips become the dominant loss mechanism. In order to suppress the steady leak of cold atoms out of the trap, we need to use a trap with a nonzero bias field, so that the atoms are trapped about a nonzero local B field minimum. There are several options for doing so. We have selected the time-orbiting potential (TOP) trap, which is described in detail below and in Chapter 4.

\subsection{Implementation}

We perform evaporative cooling using a signal generator to produce the RF radiation. The signal generator is connected to an amplifier and then to an antenna made of one loop of wire. The antenna has diameter $3.8 \mathrm{~cm}$, and delivers the RF radiation to the atoms. As I will discuss in the next chapter, we perform the evaporation while the atoms are located within a structure that will be used to make an atom waveguide once the condensate has been created. That waveguide remains off during evaporative cooling, but the structure of the device tends to shield the atoms from our RF antenna.

To help couple as much radiation to the atoms as possible, we placed the antenna directly on top of the glass vacuum chamber. Initially, having the RF antenna oriented the same way as the main B field coils was problematic, due to pickup between the two sets of coils. We eliminated that effect by placing the antenna on the side of the chamber, so that its axis was perpendicular to the axis of the main coils. Even so, we needed a $25 \mathrm{~W}$ amplifier in order to couple enough radiation to the atoms to see any evaporation. 
We used a model 325LA amplifier from Electronic Navigation Industries, with its output reading just below maximum as evaporation was initiated. We eventually shielded the entire science cell with aluminum sheeting, and placed the antenna on top of the chamber again since the antenna was now shielded from the main B field coils and was physically closer to the atoms here than from the side of the chamber.

We controlled the RF scan rate with a computer program that communicated with the RF signal generator through a GPIB. We varied the RF frequency according to the exponential ramp

$$
\nu(t)=\left(\nu_{\text {start }}-\nu_{0}\right) e^{-t / \tau}+\nu_{0}
$$

where $\nu_{\text {start }}$ is the evaporation start frequency, $\nu_{0}$ is the frequency corresponding to the bottom of the trap, and $\tau$ is the time constant of the frequency ramp. The bottom frequency $\nu_{0}$ is nonzero due to the bias field we apply to prevent Majorana losses. The bias field changes the V-shaped potential of the magnetic trap into a bowl-shaped harmonic trap, with the rounded-off bottom no longer reaching to zero energy. $\nu_{0}$ is only a few $\mathrm{MHz}$ though, and so the fact that it is nonzero is not significant until the cloud becomes fairly cold.

\subsubsection{TOP Trap}

In the TOP trap, the field zero rotates due to an oscillating bias field

$$
\mathbf{B}_{0}=B_{0}(\mathbf{x} \sin \Omega t+\mathbf{z} \cos \Omega t),
$$


and the atomic spins follow adiabatically. Recalling the quadrupole field due to the main coils,

$$
\mathbf{B}_{\text {Quad }}=B_{z}^{\prime}\left(z \mathbf{z}-\frac{x}{2} \mathbf{x}-\frac{y}{2} \mathbf{y}\right)
$$

we can solve for the time-averaged field magnitude

$$
\langle|\mathbf{B}|\rangle=\left\langle\left|\mathbf{B}_{\text {Quad }}+\mathbf{B}_{0}\right|\right\rangle
$$

In the first step the vector components of the quadrupole and bias fields are added together, and then squared. The result is

$$
|\mathbf{B}|^{2}=B_{0}^{2}+B_{z}^{\prime 2}\left(z^{2}+\frac{x^{2}+y^{2}}{4}\right)+B_{0} B_{z}^{\prime}(2 z \cos \Omega t-x \sin \Omega t) .
$$

In order to take the square root, we will use the binomial expansion

$$
(1+\epsilon)^{1 / 2} \approx 1+\frac{\epsilon}{2}-\frac{\epsilon^{2}}{8}
$$

where we will preserve only terms up to order two. We must first arrange our expression in the proper form by factoring out $B_{0}^{2}$.

$$
|\mathbf{B}|=B_{0}^{2}\left[1+\frac{B_{z}^{\prime 2}}{B_{0}^{2}}\left(z^{2}+\frac{x^{2}+y^{2}}{4}\right)+\frac{B_{z}^{\prime}}{B_{0}}(2 z \cos \Omega t-x \sin \Omega t)\right]
$$


Now we can take the square root, with

$$
\begin{aligned}
|\mathbf{B}|=B_{0}+\frac{B_{z}^{\prime 2}}{2 B_{0}}\left(z^{2}+\frac{x^{2}+y^{2}}{4}\right)+\frac{B_{z}^{\prime}}{2} & (2 z \cos \Omega t-x \sin \Omega t) \\
& -\frac{1}{8} \frac{B_{z}^{\prime 2}}{B_{0}}(2 z \cos \Omega t-x \sin \Omega t)^{2} .
\end{aligned}
$$

The final step is to average over the oscillating terms. The resulting time-averaged field magnitude of the TOP trap, to second order in the coordinates, is

$$
\langle|\mathbf{B}|\rangle=B_{0}+\frac{B_{z}^{\prime 2}}{B_{0}}\left(\frac{x^{2}}{16}+\frac{y^{2}}{8}+\frac{z^{2}}{4}\right) .
$$

Since this is a harmonic trap, we can set

$$
\mu\langle|\mathbf{B}|\rangle=\frac{1}{2} m\left(\omega_{x}^{2} x^{2}+\omega_{y}^{2} y^{2}+\omega_{z}^{2} z^{2}\right),
$$

allowing us to solve for the trap frequencies

$$
\begin{aligned}
\omega_{x} & =\left(\frac{1}{8} \frac{\mu}{m} \frac{B_{z}^{\prime 2}}{B_{0}}\right)^{1 / 2} \\
\omega_{y} & =\left(\frac{1}{4} \frac{\mu}{m} \frac{B_{z}^{\prime 2}}{B_{0}}\right)^{1 / 2} \\
\omega_{z} & =\left(\frac{1}{2} \frac{\mu}{m} \frac{B_{z}^{\prime 2}}{B_{0}}\right)^{1 / 2}
\end{aligned}
$$

The path of the orbiting field zero defines an ellipse known affectionately as the "circle of death" with radius $x_{c d}=2 B_{0} / B_{z}^{\prime}$ and $z_{c d}=B_{0} / B_{z}^{\prime}$. We can solve for the depth of the 
TOP trap by finding the energy of the circle of death

$$
U_{c d} \cong \frac{1}{2} m \omega_{z}^{2} z_{c d}^{2}=\frac{1}{4} \mu B_{0} .
$$

We can operate the bias field up to $20 \mathrm{G}$, giving a maximum depth of $340 \mu \mathrm{K}$. This is significantly colder than the temperature of the cloud at the onset of evaporative cooling, so we cannot load the TOP trap immediately. Instead we turn it on once the atoms have been sufficiently cooled.

\subsubsection{Successful Application of Evaporative Cooling}

The most difficult part of the evaporative cooling process was getting it started. At first, our efforts were impeded by poor coupling of the RF radiation to the atoms. Once we installed the $25 \mathrm{~W}$ amplifier and improved RF delivery, we observed removal of high energy atoms from our cloud by measuring the number of atoms in the trap with and without application of RF. We were unable to produce "runaway evaporation" (where the collision rate steadily increases as number decreases), however. The general rule of thumb for runaway evaporation is that the product of the lifetime $\tau$ of the atoms in the trap and the collision rate $R_{c}$ should be greater than or equal to 150 [44]. When we first attempted evaporative cooling, we were delivering to the science cell a cloud of trapped atoms with $N=6 \times 10^{8}, T=1 \mathrm{mK}$ and $R_{C}=3 \mathrm{~s}^{-1}$. According to the general rule, we then needed $\tau \sim 50 \mathrm{~s}$. We calculated $\tau$ by measuring the number of atoms in the trap for various trapping times, and fitting to an exponential function. We then found the $1 / \mathrm{e}$ 
lifetime was $\tau=26 \mathrm{~s}$, making runaway evaporation impossible.

We went back to our MOT and CMOT, in an effort to improve the initial conditions of the atomic cloud. We carefully aligned and re-optimized the MOT and CMOT, keeping the temperature as low as possible. We also paid careful attention to the power, alignment, polarization, and frequency of the optical pumping and diode repumper beams prior to loading the magnetic trap. Finally, we again ran the Titanium-sublimation pump in the science cell to remove background vapor. With these adjustments, we were able to transport $1.5 \times 10^{9}$ atoms to the science cell, at temperature $T=900 \mu \mathrm{K}$. This colder, denser cloud now had a collision rate of $R_{c}=10 \mathrm{~s}^{-1}$. However, the lifetime was still about $30 \mathrm{~s}$. While this was above the margin of the rule of thumb, we still did not observe runaway evaporation.

The pressure gauge in the science cell consistently read $4 \times 10^{-11}$ torr, so we were satisfied that the lifetime was not pressure-limited. We even attempted to raise the pressure by applying a heat gun to the walls of the vacuum chamber and running $25 \mathrm{~A}$ of current through the waveguide structure. No effect on $\tau$ was observed. Lifetime can also be limited by heating of the atoms themselves. We investigated several possible heating sources, such as current noise from the B field control panel, and glancing collisions from highly energetic trapped atoms (the so-called "Oort cloud" hypothesis [44]), but we eventually eliminated all of these possibilities.

After careful scrutiny, we attributed the poor lifetime to stray resonant light and RF fields. We devised a test whereby once the atoms were in place in the science cell, we ramped the gradient of the magnetic trap from $387 \mathrm{G} / \mathrm{cm}$ to $23 \mathrm{G} / \mathrm{cm}$ over a time period 
of $500 \mathrm{~ms}$. Since $|22\rangle$ atoms require a gradient of $15 \mathrm{G} / \mathrm{cm}$ to be supported against gravity while $|21\rangle$ atoms require $30 \mathrm{G} / \mathrm{cm}$, the reduced gradient allows us to release the $|21\rangle$ atoms and keep only the $|22\rangle$ atoms. The field remained at $23 \mathrm{G} / \mathrm{cm}$ for $100 \mathrm{~ms}$, allowing the $|21\rangle$ atoms to leave the trapping region, and then ramped back up to $387 \mathrm{G} / \mathrm{cm}$ in $500 \mathrm{~ms}$. By comparing the size of the atomic population with and without the ramp, we determined the number of atoms in the $|21\rangle$ state. This exercise revealed that after being held in the magnetic trap for $1 \mathrm{~s}, 30 \%$ of the population was in the $|21\rangle$ state and $70 \%$ was in the $|22\rangle$ state. After $30 \mathrm{~s}$ in the magnetic trap, the population evolved so that $70 \%$ of the atoms were in the $|21\rangle$ state and $30 \%$ were in the $|22\rangle$ state. It was now apparent that something was driving the $m=2 \rightarrow m=1$ transition.

This observed evolution of atomic states could be produced by either stray light, RF radiation, or a combination of the two. The two possibilities have different mechanisms. Stray resonant light will drive $|22\rangle$ atoms up into the $F^{\prime}=3$ excited state, from which some of the atoms will relax into the $|21\rangle$ state. Low frequency RF fields will drive spin state transitions, moving the population from $|22\rangle$ directly into $|21\rangle$. The key difference between the two mechanisms is that a low frequency RF field will affect both $|22\rangle$ and $|1-1\rangle$ atoms, while the resonant light will strongly interact with only the $|22\rangle$ atoms. Therefore examination of the $|1-1\rangle$ atoms should help determine the source of the population redistribution.

We measured lifetime and heating rate for both states, with results shown in Table 3.1. While the parameters for the $|1-1\rangle$ state are somewhat better than for the $|22\rangle$ state, they are still not impressive. 


\begin{tabular}{||c|c|c||}
\hline State & $\tau(\mathrm{s})$ & $\Delta T(\mu \mathrm{K} / \mathrm{s})$ \\
\hline$|22\rangle$ & 26 & 19.8 \\
$|1-1\rangle$ & 45 & 4.5 \\
\hline
\end{tabular}

Table 3.1: Lifetime and heating rate in science cell for atoms in either trapped state, when evaporative cooling did not work. The heating rate is lower in $F=1$, since the affected atoms are simply lost.

We believe that both stray light and RF fields were at play. By magnetically trapping atoms in the $|22\rangle$ state, we chose a state that strongly interacted with the main laser beams in the experiment. Since we have almost $1 \mathrm{~W}$ of laser power driving the $F=2 \leftrightarrow F^{\prime}=3$ transition, even a small amount of light from stray reflections will significantly impact the trapped population. To control this, we built a light box around our lasers and part of the beam path, with small holes just large enough to allow the beams through where necessary.

We also attempted to identify sources of $\mathrm{RF}$ radiation. We tested the Compumotor controller panel, shielded the cables from the Compumotor panel, tested dc power supplies, and ran a pickup coil over most of the optics table and surrounding area. Finally, instead of tracking down numerous possible RF sources we decided to shield the science cell. We wrapped the science cell with aluminum sheeting about $1 / 4 \mathrm{~mm}$ thick, with holes cut for laser probing. We also cut slits in the sheeting to minimize eddy currents due to the strong fields from the main B field coils. We then remeasured the lifetime and heating rate for both states, with results shown in Table 3.2. With these parameters, we achieved runaway evaporation and were able to load atoms into the TOP trap.

To continue RF evaporative cooling in the TOP trap, we cannot just begin at the same 


\begin{tabular}{||c|c|c||}
\hline State & $\tau(\mathrm{s})$ & $\Delta T(\mu \mathrm{K} / \mathrm{s})$ \\
\hline$|22\rangle$ & 83 & 2.7 \\
$|1-1\rangle$ & 162 & 1.6 \\
\hline
\end{tabular}

Table 3.2: Lifetime and heating rate in science cell for atoms in either trapped state, after construction of light box and shielding of science cell.

RF frequency that we stopped at. The bias field has changed the shape of the potential, so that the depth of the trap has changed. We can proceed to Bose-Einstein condensation by either continued RF evaporation, or ramping down the bias field so that the circle of death evaporates higher energy atoms. We found the best results through a combination of the two.

\subsection{Bose-Einstein Condensation}

We created our first Bose-Einstein condensate at 4:00 am on May 4, 2005. In this section, I present the procedure used to observe BEC.

We begin with the optimized stages for cooling and capturing atoms in the MOT chamber. Table 3.3 lists the laser state, detuning, B field gradient, and duration of each stage in the cooling and trapping process. The MOT reaches steady-state after loading for $15 \mathrm{~s}$. We then command the ADwin pulser system to begin our Bose-Einstein condensation sequence. The actual pulse sequence used is included in Appendix F. As the table shows, we first run the MOT with trapping and repump beams at full power, and then move to a CMOT with repump intensity attenuated. As described in Appendix B, we control the repump intensity by applying a bias voltage to the EOM driver. Afterwards we cool the 
atoms further with an optical molasses. We then optically pump the atoms into the $|22\rangle$ state for $1 \mathrm{~ms}$. We catch the atoms in the magnetic trap with $B^{\prime}=124 \mathrm{G} / \mathrm{cm}$, because this traps the atoms without compressing the cloud from the CMOT width. In order to protect the magnetically trapped atoms from stray resonant light, we close a mechanical shutter located in the main beam path so that all beams to the vacuum chamber are extinguished. Once the atoms are loaded into the magnetic trap, we ramp the gradient up to the maximum of $387 \mathrm{G} / \mathrm{cm}$.

\begin{tabular}{||c|c|c|c|c||}
\hline Action & Trapping/Repump & $\Delta(\mathrm{MHz})$ & B' $\left.^{\prime} \mathrm{G} / \mathrm{cm}\right)$ & Time $(\mathrm{ms})$ \\
\hline MOT & on/on full & -20 & 9 & $\sim 15000$ \\
CMOT & on/on half & -38 & 9 & 15 \\
Molasses & on/on half & -42 & 0 & 1 \\
Optical Pumping & off/off & -267 & 0 & 1 \\
B Catch & off/off & - & 124 & 50 \\
B Ramp & off/off & - & $124 \rightarrow 387$ & 200 \\
\hline
\end{tabular}

Table 3.3: Laser usage, detuning, B field gradient, and duration for each stage in the atom capture process. All detunings are referenced against the $F=2 \leftrightarrow F^{\prime}=3$ resonance.

Table 3.4 displays the number of atoms, temperature, and average density at each step. Assuming the MOT has a Gaussian density distribution, we estimate it contains $3 \times 10^{9}$ atoms at $800 \mu \mathrm{K}$. Due to the CMOT and optical molasses the cloud cools to $200 \mu \mathrm{K}$, but after the cloud is transfered to the magnetic trap, the number of atoms drops to $2 \times 10^{9}$ and due to compression the temperature rises to $900 \mu \mathrm{K}$. We consistently observe $75 \%$ transfer efficiency through the tube to the science cell.

In the science cell the atoms are evaporatively cooled. We found the evaporation stages listed in Table 3.5 resulted in successful condensation. These steps were performed 


\begin{tabular}{||c|c|c|c||}
\hline Stage & $\mathrm{N}\left(10^{9}\right)$ & $\mathrm{T}(\mu \mathrm{K})$ & $n_{0}\left(10^{10} \mathrm{~cm}^{-3}\right)$ \\
\hline MOT & 3 & 800 & 0.8 \\
CMOT & 3 & 400 & 4 \\
B Trap MOT Cell & 2 & 800 & 9 \\
B Trap Science Cell & 1.5 & 900 & 7 \\
\hline
\end{tabular}

Table 3.4: The number, temperature, and peak density of atom cloud during the MOT, CMOT and magnetic trap.

as part of the overall progression to condensation, shown in Table 3.6.

\begin{tabular}{||c|c|c|c|c|c||}
\hline Stage & $\nu_{\text {start }}(\mathrm{MHz})$ & $\nu_{\text {stop }}(\mathrm{MHz})$ & $\tau(\mathrm{s})$ & RF Power $(\mathrm{dBm})$ & $\nu_{0}(\mathrm{MHz})$ \\
\hline 1 & 50 & 30 & 12 & -13 & 0.0 \\
2 & 30 & 15 & 6 & -13 & 0.0 \\
3 & 10.4 & 4.4 & 6 & -13 & 7.6 \\
4 & 1.85 & 0.65 & 6 & -18 & 2.55 \\
5 & 0.65 & 0.18 & 3 & -18 & 2.55 \\
\hline
\end{tabular}

Table 3.5: Details of evaporative cooling at each stage. Each frequency is referenced against $\nu_{0}$, the frequency of the bottom of the trap.

Evaporative cooling begins with stages 1 and 2, which occur together. This cools the cloud below $200 \mu \mathrm{K}$, so that $k_{B} T$ of the atoms is well below the maximum TOP trap depth. Then we turn on our oscillating bias field, initiating the TOP trap at maximum bias field amplitude $B_{0}=20 \mathrm{G}$. After the atoms are loaded into the new trap, we begin stage 3 of evaporation. There is a discontinuity between the stop frequency of stage 2 and the start frequency of stage 3 because initiation of the TOP trap has changed the potential experienced by the atoms. Therefore, the RF level appropriate for the next cut has also changed. After stage 3 , we ramp the TOP field down to $B_{0}=4 \mathrm{G}$, making a 


\begin{tabular}{||c|c|c|c|c||}
\hline Stage & $\mathrm{N}$ & $\mathrm{T}(\mu \mathrm{K})$ & $\mathrm{R}_{c}\left(\mathrm{~s}^{-1}\right)$ & PSD \\
\hline Before Evap & $1.5 \times 10^{9}$ & 900 & 11 & $7 \times 10^{-8}$ \\
Evap 1 & $5 \times 10^{8}$ & 400 & 28 & $1 \times 10^{-6}$ \\
Evap 2 & $1.5 \times 10^{8}$ & 180 & 65 & $2 \times 10^{-5}$ \\
Load TOP & $9 \times 10^{7}$ & 85 & 10 & $2 \times 10^{-6}$ \\
Ramp TOP & $4 \times 10^{7}$ & 40 & 20 & $3 \times 10^{-5}$ \\
Evap 3 & $2 \times 10^{7}$ & 18 & 20 & $1 \times 10^{-4}$ \\
Ramp TOP & $4 \times 10^{6}$ & 7 & 52 & $2.2 \times 10^{-3}$ \\
Evap 4 & $5 \times 10^{5}$ & 1.4 & 37 & $3.8 \times 10^{-2}$ \\
Evap 5 & $2 \times 10^{4}$ & 0.2 & & $>2$ \\
\hline
\end{tabular}

Table 3.6: Evaporative cooling sequence raises phase space density (PSD) to achieve condensation.

tighter, shallower trap. This once again changes the potential, and so evaporation stages 4 and 5, which also occur together, again do not start where stage 3 left off. In stage 3 the frequency of the trap bottom starts to become important, as we begin to reach near enough to the bottom of the well to discern that it is not actually at $0 \mathrm{MHz}$. Each start and stop frequency is referenced against the frequency of the trap bottom, so that in stage 1 the actual applied RF frequency is $50+0=50 \mathrm{MHz}$, while in stage 5 the applied value is $0.65+2.55=3.2 \mathrm{MHz}$.

Note that the time constant and RF intensity are lowered as evaporation proceeds. The rapidly increasing collision rate allows the rethermalization rate to increase, reducing the time required before the next cut can be made. As the average energy of the atoms becomes commesurate with the spectrum of the RF field, the RF knife dulls due to power broadening. Also, the efficiency of our RF antenna increases at lower frequencies. To make the precise cuts we require, the intensity of the RF is reduced as we continue evaporating. 
We experimented with both low frequency $(\sim \mathrm{MHz})$ evaporation between Zeeman levels, and high frequency $(\sim 6.8 \mathrm{GHz})$ evaporation between the $F=2$ and $F=1$ ground states. While we ended up using the low frequency evaporation to make condensates, the only substantial obstacle to using the high frequency evaporation is that we had difficulty supplying enough RF power. At that time we were using a $1 \mathrm{~W}$ amplifier, and we have not attempted high frequency evaporation since we upgraded to the $25 \mathrm{~W}$ amplifier. Note the high-frequency evaporation requires a microwave antenna and waveguide instead of a simple single-loop antenna.

While the waveguide that I will discuss in the next chapter was always intended for condensates of $|22\rangle$ atoms, we originally attempted to use atoms in the $|1-1\rangle$ state for evaporative cooling, primarily because many groups have found this state easier to work with. The plan was to evaporate with $|1-1\rangle$ atoms, and then apply an RF field to transfer the population to $|22\rangle$ after the condensate was formed. We eventually decided to evaporate with $|22\rangle$ atoms, since we loaded them into the magnetic trap more efficiently and when we were struggling with evaporative cooling we used every means possible of increasing $N$ and $R_{c}$. However, we did observe a longer lifetime for the $|1-1\rangle$ state, and now that we have achieved success with evaporative cooling there is no reason why we could not try to make a condensate of $|1-1\rangle$ atoms in the future.

We observe condensation of atoms in the $|22\rangle$ state with approximately $2 \times 10^{4}$ atoms at $200 \mathrm{nK}$. Figures 3.6 and 3.7 show the onset of condensation. The frequency of the applied $\mathrm{RF}$ is $2.95 \mathrm{MHz}$ in the first image, where we have a cold, uncondensed cloud just above $200 \mathrm{nK}$. In the next image, the RF has been scanned down to $2.90 \mathrm{MHz}$, and we 
observe the formation of a tiny condensate at the center of the thermal cloud. Finally when the $\mathrm{RF}$ reaches $2.77 \mathrm{MHz}$, we observe most of the remaining thermal atoms join the condensate.

Figure 3.8 depicts temperature and phase space density as the number of atoms is decreased through evaporative cooling. Notice the drop in phase space density as the TOP trap is loaded, at $N \sim 1 \times 10^{8}$ atoms. This signifies an inefficiency in the process of transferring the atoms to the TOP trap. Certainly it would be preferable to simply begin evaporation within the TOP trap and avoid such a transfer altogether, but the maximum depth of our TOP trap is well below the average energy of the atoms prior to evaporation. Furthermore, we cannot translate the TOP trap between the two vacuum chambers because, as we shall see in Chapter 4, the oscillating bias field is produced using part of the waveguide structure.

We believe the problem of loading the TOP trap is due to non-adiabatic expansion of the atom cloud. We switch the TOP trap on quickly at full strength, for obviously if we were to ramp the TOP fields up the field zero would slowly pass through the cloud and devastate the population of the trap. But by initiating the TOP trap quickly, we make a sudden change to the confining potential. The atoms relax into the new trap through a non-adiabatic expansion of the cloud, reducing the phase space density. Some possible solutions to this problem include laser cooling the atoms in the science cell, or perhaps rapidly increasing $B^{\prime}$ during the transfer. This issue will likely be explored in the future, since we believe that if it were resolved we could produce condensates with $N \geq 10^{5}$ atoms. 


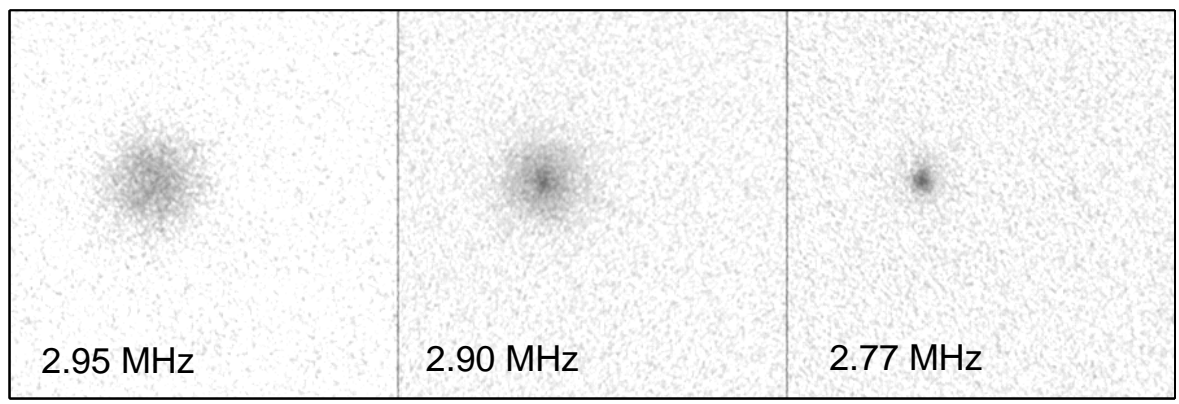

Figure 3.6: Condensate formation. The first image shows the cloud just prior to the onset of Bose-Einstein condensation. As the temperature is lowered, the second image shows the condensate begin to form, and in the third image most of the atoms have joined the condensate. The frequencies refer to the RF applied at each stage.

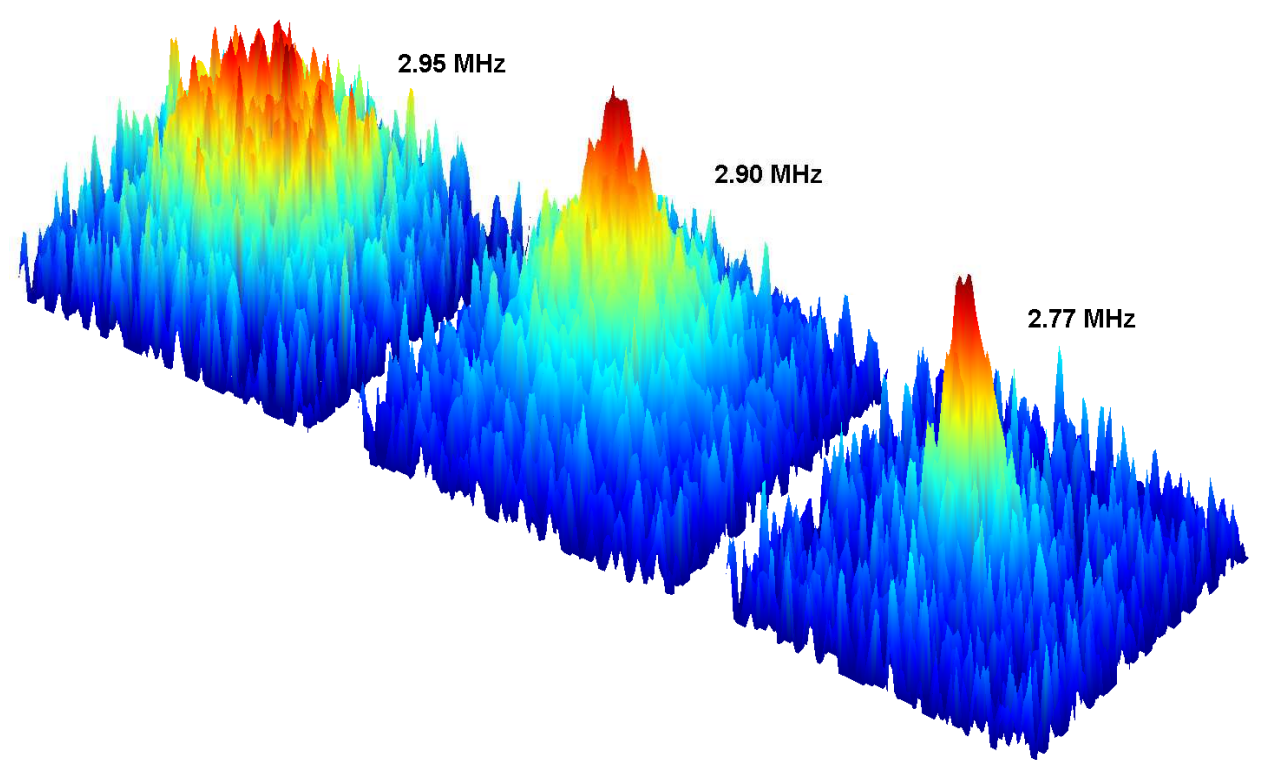

Figure 3.7: Condensate formation. Each frame is a density plot of the data in Figure 3.6. The frequencies refer to the RF applied at each stage. As the condensate forms, a sharp high-density peak forms at the center of the cloud. As the temperature is gradually reduced, more atoms populate the ground state, joining the condensate. 

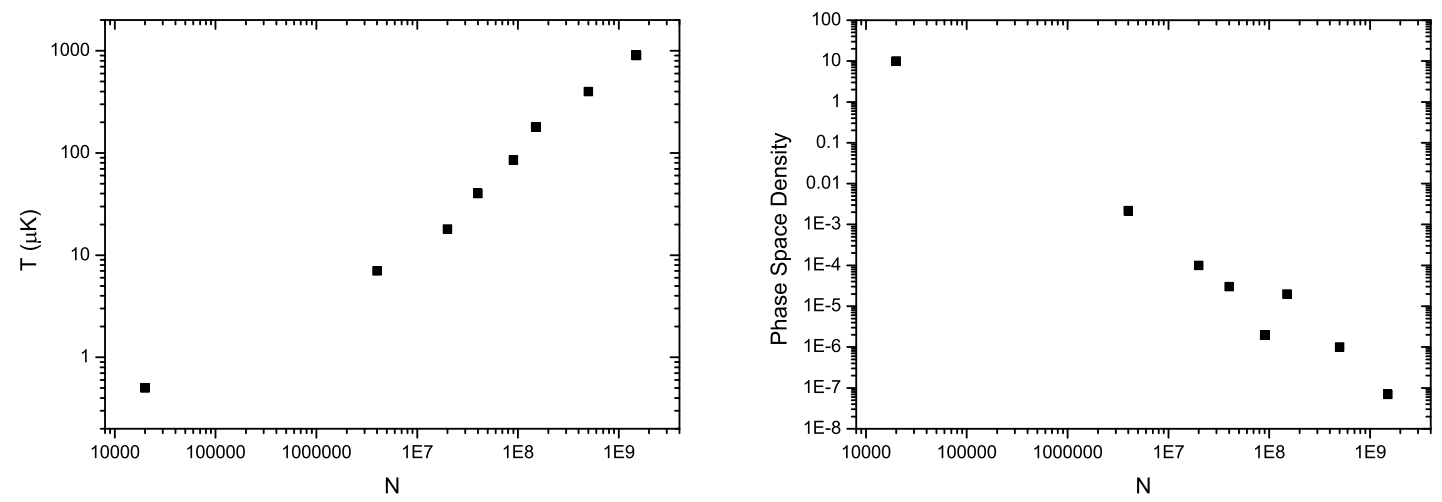

Figure 3.8: Progression of evaporative cooling. Temperature decreases and phase space density (PSD) increases as highest energy atoms are removed from cloud. Due to the drop in PSD at $N=1 \times 10^{8}$, as the TOP trap is loaded, our condensates are somewhat smaller than typical. Improved efficiency while loading the TOP trap should produce higher condensate number.

\subsection{Imaging}

We collect data about the atomic cloud by taking pictures of it and then processing the images. Since our condensates are tens of micrometers in size, we require a camera with high magnification and resolution. As a further complication, our ${ }^{87} \mathrm{Rb}$ condensate scatters light at $780 \mathrm{~nm}$, which is just outside the visible spectrum. Also, the number of photons scattered by $10^{4}$ atoms from a few hundred $\mu \mathrm{W}$ of laser power is small, and so there is not much light to be detected. As if these concerns were not enough, the imaging system must also be versatile enough to view the MOT, CMOT, magnetic trap, and various steps in evaporation.

There are two primary methods of viewing a cloud of atoms. Fluorescence imaging captures the light scattered by the atoms, producing a bright image of the atoms against a dark background. Absorption imaging involves shining a laser through the atoms, directly 
into the camera. The atoms appear as a dark spot against a bright background, so that we are essentially seeing the shadow cast by the cloud. Both methods are used at various points in our experiment.

\subsubsection{Fluorescence}

Atoms trapped in the MOT undergo spontaneous emission and radiate $780 \mathrm{~nm}$ light in all directions. A photodiode will detect a portion of that light, depending on the solid angle subtended by the detector. We use a series of lenses to collect as much light as possible. We then use the measured photodiode current to calculate the number of atoms, as given by

$$
N=\frac{4 \pi(\text { photodiode current })}{(\text { solid angle })(\text { responsivity })(\text { energy of photon }) R_{s}}
$$

where the solid angle is $\pi R^{2} / d^{2}$ and $R$ is the radius of the collection lens at distance $d$ from the atoms. $R_{s}$ is the scattering rate given by Eq. (2.6), the energy of a photon is $\hbar \omega=h c / \lambda$, and the responsivity of our photodiode is measured at $0.52 \mathrm{~A} / \mathrm{W}$. Figure 3.9 shows our fluorescence imaging system. Our collection lens is placed as close to the window into the vacuum chamber as we could get it (about $450 \mathrm{~mm}$ from the atoms), and has focal length $150 \mathrm{~mm}$ and diameter 2 inches. The captured light is passed to a beamsplitter, which directs a portion of the signal into a stronger $(f=25 \mathrm{~mm})$ lens which then focuses the signal onto the face of a photodiode with $13 \mathrm{~mm}^{2}$ active area. The photodiode signal is our primary indicator of MOT performance. The rest of the light passes to an infrared camera, with its output displayed on a television monitor. While we can make quick estimates of MOT health by observing apparent size, shape 


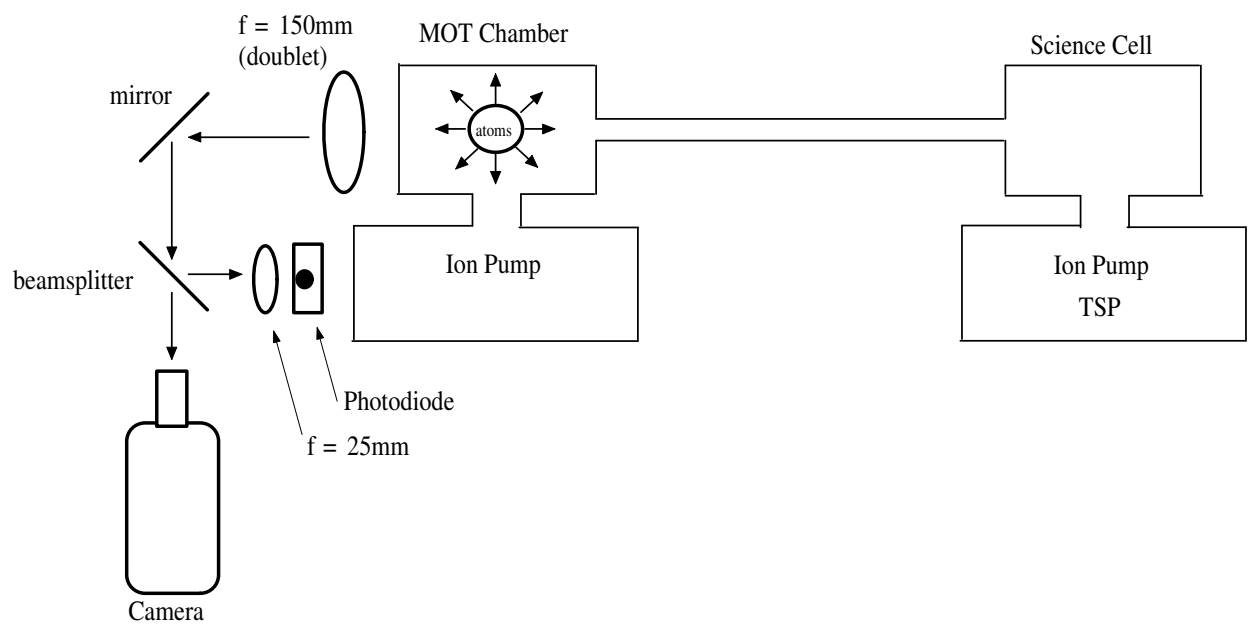

Figure 3.9: Fluorescence imaging system. A large diameter collection lens sits just outside the MOT chamber, directing fluorescence from the atoms into the imaging system. A beamsplitter divides the signal between an infrared camera and a photodiode. The output of the camera is displayed on a television monitor, allowing us to verify at any given moment that the MOT is working. The photodiode signal is displayed on an oscilloscope and an analog meter. There is a small diameter, strong lens just prior to the photodiode, that focuses the light to fit onto the $13 \mathrm{~mm}^{2}$ face of the photodiode.

and brightness of the image on the tv monitor, the photodiode signal is preferred for quantitative comparison.

Fluorescence imaging is simple to implement. It requires only a clear view of the atoms and a camera capable of detecting light at $780 \mathrm{~nm}$. Merely running the MOT allows fluorescence images to be taken continuously. However, fluorescence images are generally imprecise. It is difficult to calibrate the photodiode, primarily because of issues with the scattering rate. $R_{s}$ is affected by a number of mechanisms, including power broadening, line broadening and optical thickness. Additionally, to calculate $R_{s}$ accurately we must know not only the polarization of the light but also the distribution of atomic spin states, which is we do not know exactly. For these reasons, we tend to use fluorescence imaging as a "quick and dirty" tool, but we rely more on absorption imaging for firm data about 
the atoms.

\subsubsection{Absorption}

Absorption imaging works by detecting the absence of scattered photons, in direct contrast to fluoresence imaging. As illustrated by Figure 3.10, we use a system of lenses and mirrors to focus a CCD camera on the plane of the atoms. A probe laser beam is sent through the atoms, into the imaging optics, and is incident upon the CCD window. The camera thus looks directly into the beam, and the atoms appear as a dark spot within the image of the beam. We used an Apogee model AP47 CCD camera system. We image the condensate after it is released from the trap, as it falls away from the camera. We allowed the cloud to expand ballistically so that the density was reduced until the absorption coefficient of the image was typically between 0.5 and 2.0. For different points in the evaporative cooling process, this required expansion times of $1 \mathrm{~ms}, 10 \mathrm{~ms}$, or $20 \mathrm{~ms}$. The cloud can fall up to $2 \mathrm{~mm}$ away from the camera during this expansion, and unless our imaging system can compensate some of the images will be blurry. We placed one of the lenses on a micrometer, and adjusted it to bring images at each different expansion time into focus. We control the magnification by selecting either a $2 \mathrm{X}$ or $5 \mathrm{X}$ microscope objective.

To produce one calibrated composite image of the atoms, we capture three raw images. The first image, captured with no atoms or lasers present, is designated the background image. It provides a measure of the level of ambient and leakage light through the system, as well as the CCD offset signal. We then capture an image of the probe beam shining through the atom cloud, which we call the "atoms" image. Finally, we image the probe 


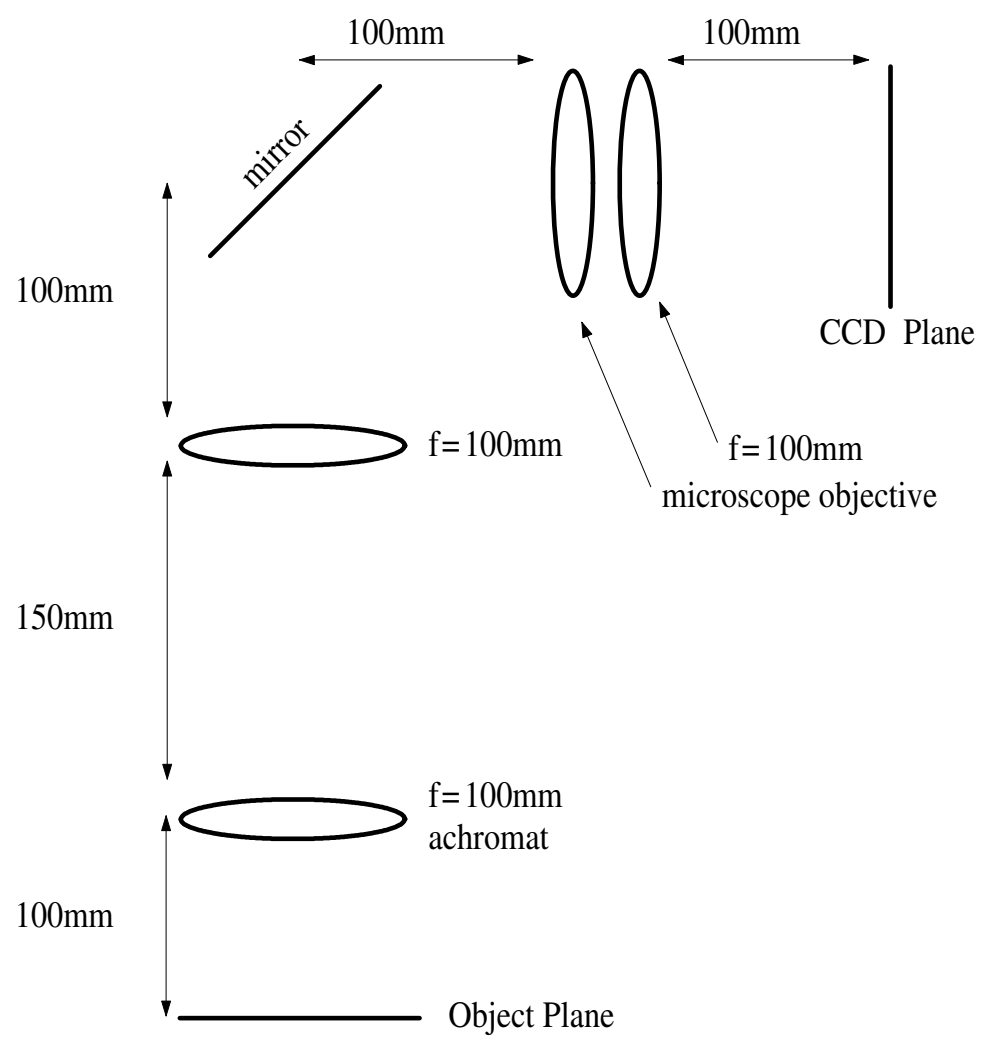

Figure 3.10: Arrangement of optics in absorption imaging system. We have objectives at $2 \mathrm{X}$ and $5 \mathrm{X}$ magnification. The longer we allow the condensate to fall prior to imaging, the farther the object plane will be from the first lens. To compensate, we placed the second lens from the object plane on a micrometer so that the position of this lens can be adjusted as needed to focus the image.

beam again, but with no atoms present. We call this the "no atoms" image. We can calculate the absorption $\alpha(x, y)$ in the plane of the image as

$$
e^{-\alpha}=\frac{(\text { atoms image }- \text { background image })}{(\text { no atoms image }- \text { background image })} \text {. }
$$

It is important that the laser intensity and the exposure time are the same in the "atoms" and "no atoms" images.

We obtain clear absorption images with short laser flashes (about $30 \mu \mathrm{s}$ ) and low 
laser intensity (a few $\mu \mathrm{W}$ per $\mathrm{cm}^{2}$ ), making this method more reliable than fluorescence imaging. The calibration is also easier to perform, since we simply measure the background light level and subtract it out. We use absorption images to calculate quantities like atom number, temperature, density, collision rate, and phase space density.

\subsubsection{Calculations}

There are four distinct conditions in which we analyze the atomic cloud. These are the MOT/CMOT, the quadrupole trap, the TOP trap, and the Bose-Einstein condensate. The density distribution is different in each case, which must be reflected in the equations used to find the quantities of interest.

\section{MOT/CMOT}

For atoms in the MOT and CMOT, we were most interested in the number of atoms $N$ and their average temperature T. We often used fluorescence imaging and Eq. (3.30) to determine $N$, although we also arranged an absorption imaging system for better accuracy. This system included a probe beam that traveled along the axis of the chamber, from the science cell through the tube to the MOT cell and then into the camera used to monitor the MOT. The absorption images were difficult in the MOT, however, since the width of the cloud was comparable to the width of the probe beam. Using either type of imaging, we obtained the temperature of the cloud by turning off the trap and allowing the cloud

to expand ballistically. The rate of expansion is directly related to the temperature of the 
cloud, since

$$
\frac{1}{2} k T=\frac{1}{2} m\left\langle v^{2}\right\rangle
$$

We analyze the images by fitting to the Gaussian function $\frac{1}{\sqrt{\pi} w} e^{-r^{2} / w^{2}}$. The Gaussian width $w$ is related to the mean square radius as $w^{2}=2\left\langle r^{2}\right\rangle$. We can then take the w's from a series of images taken at different expansion times, and fit to the function $w^{2}=\alpha+\xi t^{2}$. Taking only the second order term, we can solve for the mean square velocity as $\xi=2\left\langle r^{2}\right\rangle / t^{2}=2\left\langle v^{2}\right\rangle$. By inserting this relationship into Eq. (3.32), we can solve for the temperature

$$
T=\frac{m \xi}{2 k}
$$

\section{Quadrupole Trap}

Fluorescence imaging is difficult in the magnetic trap due to the high field gradients and resultant Zeeman shifts. Once the atoms are loaded into the magnetic trap, we use absorption imaging exclusively. Given the spatial absorption profile of the image and the shape of the trapping potential, we can directly calculate atom number and temperature.

The absorption coefficient $\alpha$ of the image is related to the spatial density $n$ of the cloud as

$$
\alpha(x, y)=\sigma \int n \mathrm{~d} z
$$

where $\sigma$ is the light scattering cross section, $x y$ is the plane of the image and $z$ is the axis along which we are looking. We will address $n$ first, and then $\sigma$.

As discussed in Section 3.1, the general form for the density distribution is $n(\mathbf{r})=$ 
$n_{0} e^{-V(\mathbf{r}) / k T}$. For a quadrupole trap the shape of the potential is given by

$$
V=\mu|\mathbf{B}|=\mu B_{z}^{\prime} \sqrt{z^{2}+\frac{x^{2}+y^{2}}{4}},
$$

and the peak density is

$$
n_{0}=\frac{N}{32 \pi r_{1}^{3}}
$$

The quadrupole potential leads to a complicated integral for $\alpha$. This integral is not solvable analytically, making use of a fitting routine problematic. However it can be wellapproximated by a Gaussian curve, as shown below. Therefore, we estimate the number, temperature and density by fitting the images of our cloud to a Gaussian distribution.

Accordingly, we assume a Gaussian density distribution, so that $n(\mathbf{r})=n_{0} \exp \left(-\frac{x^{2}}{w_{x}^{2}}-\right.$ $\left.\frac{y^{2}}{w_{y}^{2}}-\frac{z^{2}}{w_{z}^{2}}\right)$. Integrating through all space with $N=\int n(\mathbf{r}) \mathrm{d}^{3} r$, we find peak density

$$
n_{0}=\frac{N}{\pi^{3 / 2} w_{z} w_{y} w_{z}} .
$$

Now we solve for the integral of the density distribution

$$
\begin{aligned}
\int n \mathrm{~d} z & =n_{0} e^{-\frac{x^{2}}{w_{x}^{2}}-\frac{y^{2}}{w_{y}^{2}}} \int e^{\left(-z^{2} / w_{z}^{2}\right)} \mathrm{d} z \\
& =\frac{N}{\pi^{3 / 2} w_{x} w_{y} w_{z}} e^{-\left(\frac{x^{2}}{w_{x}^{2}}+\frac{y^{2}}{w_{y}^{2}}\right)} \sqrt{\pi} w_{z} \\
& =\frac{N}{\pi w_{x} w_{y}} e^{-\left(\frac{x^{2}}{w_{x}^{2}}+\frac{y^{2}}{w_{y}^{2}}\right)} .
\end{aligned}
$$

To complete our calculation of $\alpha$, we must also find the light scattering cross section. 
In an unbroadened system, the cross section is given by

$$
\sigma=\frac{\sigma_{0} \Gamma^{2}}{\Gamma+4 \Delta^{2}}
$$

where $\Delta$ is the detuning, $\Gamma$ is the natural linewidth of $2 \pi \times 6 \mathrm{MHz}$, and $\sigma_{0}=6 \pi\left(\frac{\lambda}{2 \pi}\right)^{2} \times$ $\bar{c}$. Here the average coupling $\bar{c}$ reflects the coupling strengths of the different Zeeman sublevels, as depicted in Figure A.1. Assuming the atoms are depolarized during the probe, which is linearly polarized, $\bar{c}=\frac{1}{5}\left[\frac{1}{3}+\frac{8}{15}+\frac{3}{5}+\frac{8}{15}+\frac{1}{3}\right]$, so that $\sigma_{0}=2.8 \pi\left(\frac{\lambda}{2 \pi}\right)^{2}$. In reality, our system experiences line broadening, with observed linewidth $\Gamma^{\prime}$. Conservation of the oscillator strength $\int \sigma \mathrm{d} \omega$ gives a reduced cross section $\sigma_{1}$, with $\sigma_{0} \Gamma=\sigma_{1} \Gamma^{\prime}$. The total cross section is then

$$
\sigma=\frac{\sigma_{1} \Gamma^{2}}{\Gamma^{\prime 2}+4 \Delta^{2}}=\frac{\sigma_{0} \Gamma \Gamma^{\prime}}{\Gamma^{\prime 2}+4 \Delta^{2}}
$$

All that remains in order to find $\alpha$ is to put Eq.'s (3.38) and (3.40) together, so that

$$
\alpha_{0}=\int \sigma n \mathrm{dz}=\frac{\sigma_{0} \Gamma \Gamma^{\prime}}{\Gamma^{\prime 2}+4 \Delta^{2}} \frac{N}{\pi w_{x} w_{y}} .
$$

Rearranging,

$$
N=\frac{4 \pi^{2} \times \alpha_{0}\left(\Gamma^{2}+4 \Delta^{2}\right) w_{x} w_{y}}{2.8 \lambda^{2} \Gamma \Gamma^{\prime}}
$$

where $w_{x}$ and $w_{y}$ are the widths of the cloud in the plane of the image, $\Gamma^{\prime}$ is measured by probing the atoms from one edge of the resonance to the next and then fitting the response to a Lorentzian and finding the FWHM of the fit, and $\alpha_{0}$ is the peak absorption coefficient. We measured $\Gamma^{\prime}=2 \pi \times 12 \mathrm{MHz}$ in the MOT, with $\Gamma^{\prime}$ approaching $2 \pi \times 6 \mathrm{MHz}$ 
as the cloud cools.

To find the temperature, we need to relate the Gaussian fit to the actual quadrupole trap distribution. The exact form of the absorption coefficient is

$$
\alpha(x, y)=\sigma n_{0} \int_{-\infty}^{\infty} e^{-\frac{\mu B^{\prime}}{k T}\left(z^{2}+\frac{x^{2}+y^{2}}{4}\right)^{1 / 2}} \mathrm{~d} z
$$

Substituting $u=z \frac{2}{\sqrt{x^{2}+y^{2}}}$, the absorption is now

$$
\begin{aligned}
\alpha & =\sigma n_{0} \frac{\sqrt{x^{2}+y^{2}}}{2} \int_{-\infty}^{\infty} e^{-\frac{\mu B^{\prime}}{k T} \frac{\sqrt{x^{2}+y^{2}}}{2} \sqrt{u^{2}+1}} \mathrm{~d} u \\
& =\sigma n_{0} \frac{\rho}{2} \int_{-\infty}^{\infty} e^{-\frac{\mu B^{\prime}}{2 k T} \rho \sqrt{u^{2}+1}} \mathrm{~d} u
\end{aligned}
$$

Here we have defined $\rho=\sqrt{x^{2}+y^{2}}$. At this point we can collect terms by introducing

$$
\beta=\frac{\mu B^{\prime} \rho}{2 k_{B} T}=\frac{\rho}{2 r_{1}} .
$$

Now our equation for $\alpha$ is of the form

$$
\alpha(\beta)=\sigma n_{0} 2 r_{1} f(\beta)
$$

for

$$
f(\beta)=\beta \int_{-\infty}^{\infty} \mathrm{d} u e^{-\beta \sqrt{u^{2}+1}} .
$$

We plotted $f(\beta)$ against $\beta$, and fit to a Gaussian to obtain $w_{\beta}=1.8$ as shown in Figure 3.11. Consequently, $w_{\rho}=2 r_{1} w_{\beta}=3.6 r_{1}$ and by decomposing $r_{1}$ we find the temper- 


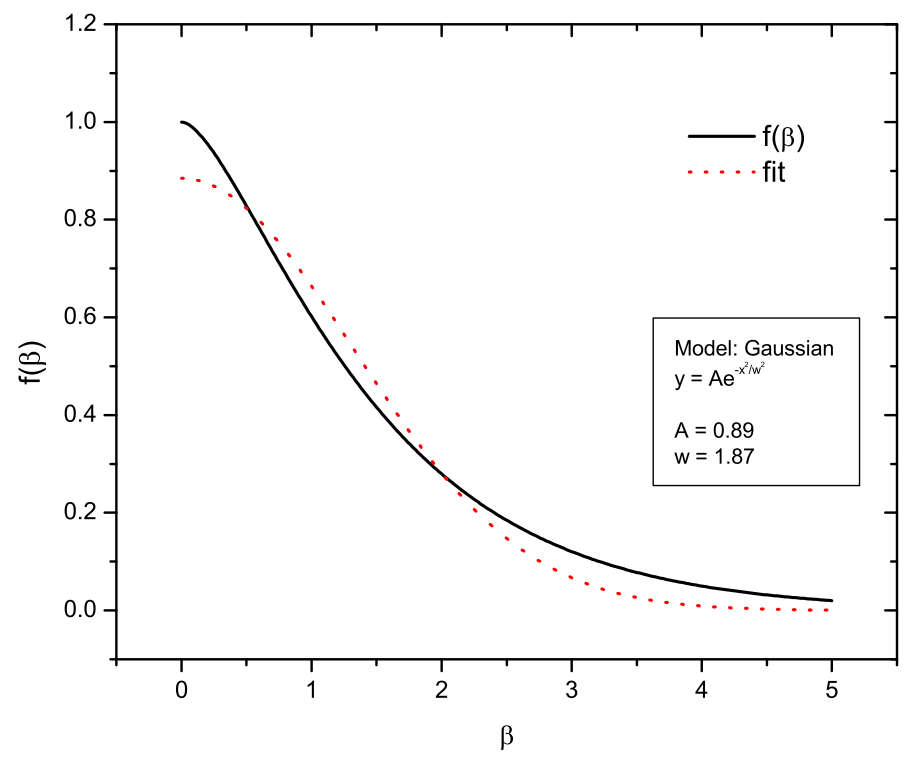

Figure 3.11: In order to find the temperature of the cloud, we must relate the Gaussian fit to the actual quadrupole distribution. The solid line is a numerical plot of $f(\beta)$, and the dotted line is a Gaussian fit to that plot. The width of the Gaussian is 1.9. For historical reasons, we use 1.8 in our calculations.

ature is

$$
T=\frac{\mu B^{\prime} w_{\rho}}{3.6 k}
$$

where $w_{\rho}$ is the measured width of the cloud image. We estimated $w_{\rho}=\left(w_{x}+w_{y}\right) / 2$.

Another quantity of interest is the interatomic collision rate $R_{c}=\bar{n} \sigma \bar{v}$, where $\bar{v}=$ $\sqrt{k T / m}$ and $\sigma=8 \pi a^{2}$. The scattering length of ${ }^{87} \mathrm{Rb}$ is $a=5.77 \mathrm{~nm}$. Using Eq. (3.37) for the density of the cloud, the collision rate is

$$
R_{c}=\frac{a^{2}}{32 \sqrt{m}}\left(\mu B_{z}^{\prime}\right)^{3} N(k T)^{-5 / 2} .
$$




\section{TOP Trap}

These calculations must be modified when the atoms are moved from the quadrupole trap into the TOP trap, because the trapping potential changes. The new potential is harmonic, with trap frequencies according to Eq. (3.28) as derived in Section 3.3.1.

The atom cloud assumes a Gaussian shape in the TOP trap. Therefore, we can once again use Eq. (3.42) for the number of atoms. The harmonic form of the density is

$$
n(\mathbf{r})=n_{0} e^{-\frac{m}{2 k T}\left[\omega_{x}^{2} x^{2}+\omega_{y}^{2} y^{2}+\omega_{z}^{2} z^{2}\right]}
$$

As a result, the widths of the cloud along all three coordinate axes are

$$
w_{x}^{2}=16 \frac{k T B_{0}}{\mu B_{z}^{\prime 2}} \quad w_{y}^{2}=8 \frac{k T B_{0}}{\mu B_{z}^{\prime 2}} \quad w_{z}^{2}=4 \frac{k T B_{0}}{\mu B_{z}^{\prime 2}} .
$$

The peak density is again

$$
n_{0}=\frac{N}{\pi^{3 / 2} w_{x} w_{y} w_{z}},
$$

and the average density is

$$
\bar{n}=\frac{1}{N} \int n^{2}(\mathbf{r}) \mathrm{d}^{3} r=\frac{N}{(2 \pi)^{3 / 2} y_{0}^{3}}=\frac{n_{0}}{2^{3 / 2}} .
$$

The temperature calculation begins again with the absorption coefficient

$$
\alpha(x, y)=\int \sigma n \mathrm{~d} z=\frac{N \sigma_{L}}{\pi w_{x} w_{y}} e^{-\left(\frac{x^{2}}{w_{x}^{2}}+\frac{y^{2}}{w_{y}^{2}}\right)},
$$


which we have found more easily than before since the integral can now be solved analytically. Since we have a Gaussian distribution, we can solve Eq. (3.51) to find the temperatures in the $x$ and $y$ directions.

$$
T_{x}=\frac{\mu B_{z}^{\prime 2} w_{x}^{2}}{16 k B_{0}} \quad T_{y}=\frac{\mu B_{z}^{\prime 2} w_{y}^{2}}{8 k B_{0}}
$$

The average temperature is then

$$
T=\frac{\mu B_{z}^{\prime 2}}{16 k B_{0}}\left(w_{y}^{2}+\frac{1}{2} w_{x}^{2}\right)
$$

Finally, using the Gaussian widths and $\sigma=8 \pi a^{2}$, we can find the collision rate in the TOP trap.

$$
R_{c}=\bar{n} \sigma \bar{v}=\frac{a^{2}}{\sqrt{\pi m} k T} N\left(\frac{\mu B_{x}^{\prime 2}}{B_{0}}\right)^{3 / 2}
$$

\section{Bose-Einstein Condensate}

Once the condensate forms, the density of the cloud is usually described with a ThomasFermi distribution. Within the cloud, the density is given by

$$
n_{c}(\mathbf{r})=\frac{15 N \bar{\omega}}{8 \pi}\left(\frac{m}{2 \mu}\right)^{3 / 2}\left(1-\frac{x^{2}}{x_{0}^{2}}-\frac{y^{2}}{y_{0}^{2}}-\frac{z^{2}}{z_{0}^{2}}\right),
$$

where $\bar{\omega}=\omega_{x} \omega_{y} \omega_{z}$ is the product of the trap frequencies and $\mu$ is the chemical potential. The surface $n_{c}=0$ defines the boundary of the condensate, beyond which $n_{c}$ remains zero. To find the number of atoms in the condensate, we use the same integral $N=\int n(\mathbf{r}) \mathrm{d}^{3} r$ 
as before.

In the actual Thomas-Fermi model, the widths $x_{0}, y_{0}$ and $z_{0}$ are determined by the number of atoms, the trap potential, and the scattering length [20]. However, for a condensate that has been allowed to freely expand before imaging, the relationship is complicated. For simplicity, we treat the widths as independent fit parameters. We estimated the number as

$$
N_{c}=\frac{2 \pi}{5} \frac{\alpha_{0}}{\sigma} x_{0} y_{0}
$$

where $\sigma$ is the light cross section, $\alpha_{0}$ is the peak absorption coefficient and $x_{0}, y_{0}$ are the widths of the image of the cloud. For partially condensed clouds, we applied a double fit using this Thomas-Fermi estimate for the condensate number $N_{c}$ and the TOP trap fit to find the number of thermal atoms $N_{t h}$. The condensate fraction is then $N_{c} / N_{t h}$. More detailed development of the Thomas-Fermi distribution can be found in [33] and [20]. 


\section{Chapter 4}

\section{Atom Waveguide}

Atom interferometry with Bose-Einstein condensates has drawn a considerable amount of interest due to the potential for high-precision measurements [14]. In this chapter I will discuss the merits of condensate interferometry, the design of our device, and its successful operation.

\subsection{Atom Interferometry}

Interferometry is the study of interference. Figure 4.1 shows a typical laser interferometer. A light source, usually a laser, supplies photons that encounter a beamsplitter which coherently splits the light into two distinct wavepackets. Each wavepacket follows its own route through the interferometer, developing a phase

$$
\phi=\frac{2 \pi}{\lambda} \int_{s_{1}}^{s_{2}} n(s) \mathrm{d} s
$$

where $n$ is the index of refraction of the medium, $s$ is the distance that the light travels through the medium, and $\lambda$ is the wavelength of the light. Any difference in $n$ or $s$ between the two arms of the device results in the acquisition of a differential phase between the two 


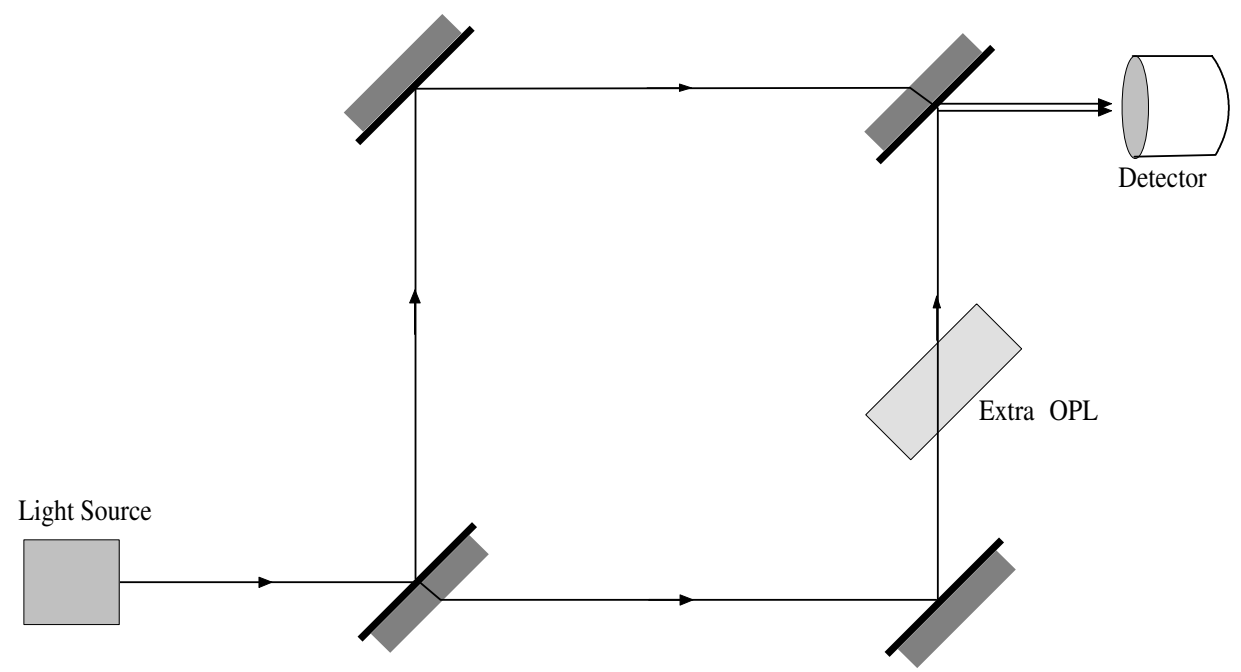

Figure 4.1: Typical laser interferometer (Mach-Zender). The input beam is incident upon a beamsplitter, which sends the two output beams into the arms of the device. Once arm includes more optical path length (OPL) than the other, so that a relative phase develops between the two beams. A final beamsplitter recombines the beams, which are then analyzed at the detector. The relative phase difference results in an interference pattern at the detector.

beams of photons. The photons are then coherently recombined at another beamsplitter and analyzed. The observed interference signal depends on the relative phase between the two paths.

For example, if the Mach-Zender interferometer in Figure 4.1 is rotated counterclockwise, the upper path is shortened and the lower path is lengthened. This is known as the Sagnac effect [58], and occurs in any planar interferometer when the device is rotated in the plane of the optics. The Sagnac phase is given by [63]

$$
\phi=\frac{4 \pi \Omega A}{\lambda v},
$$

where $\Omega$ is the rotation rate, $A$ is the area enclosed by the arms of the device, and $v$ is the velocity of the interfering field. The first ring laser gyroscope based on the Sagnac effect 
was developed in 1963 [38], and Sagnac interferometers are now widely used for inertial sensing.

Atoms can also be used for interferometry. An atom interferometer operates in much the same way as a photon interferometer. A source of atoms interacts with a coherent atom beamsplitter, which splits the atomic wavefunctions so that there are two separate routes through the device. As the atoms travel along either path, they develop a differential quantum phase. When the atomic wavefunctions are coherently recombined at the next beamsplitter, this relative phase is determined through observation of interference. Such a device highlights the wavelike nature of atoms. This is also the only way that quantum phase can be measured, since we cannot directly observe the atomic wavefunction.

Atoms have been used to measure rotations, with [28] standing as the most sensitive such device on record. Another example of atom interferometry is a gravity sensor. If we send a beam of atoms through a device such as shown in Figure 4.1, with interferometer arms oriented vertically, the atoms will accumulate phase due to their gravitational potential energy

$$
\phi=\frac{1}{\hbar} \int m g \Delta z \mathrm{~d} t
$$

The atoms traveling through the upper arm are a greater distance $z$ from the earth than the atoms in the lower arm. By measuring the differential phase between the two arms, we can find the potential energy difference and then calculate the value of the gravitational constant $g$ at that location. Such a gravity sensor might be used to search for oil deposits, by detecting local variations in $g$ that indicate a low density region in the earth's surface.

Atom interferometry is well-suited for sensor applications since atoms are affected by 
phenomena that photons are not, such as electric and magnetic fields. Atoms are especially attractive for inertial sensing, given their considerable inertia compared to photons. This advantage is demonstrated through calculation of the Sagnac phase for both particles. The optical field has wavelength $\lambda=\frac{2 \pi c}{\omega}$, while the atomic wavelength is given by $\lambda=\frac{h}{m v}$ where $m$ is the mass of an atom and $v$ is the atomic velocity. If we take the ratio of the phase sensitivity $d \phi / d \Omega$ for the two types of particles, we find a relative phase sensitivity of

$$
\frac{\phi_{\text {atom }}}{\phi_{\text {photon }}}=\frac{\lambda_{\text {photon }} v_{\text {photon }}}{\lambda_{\text {atom }} v_{\text {atom }}}=\frac{2 \pi c^{2}}{\omega} \frac{m v}{h v}=\frac{m c^{2}}{\hbar \omega} .
$$

For visible wavelength light and middle-weight atoms, this ratio is on the order of $10^{11}$, indicating the fundamental limit on the sensitivity of an atom-based device substantially exceeds that of its photon-based counterpart.

This advantage is reduced for a number of reasons, most significantly because atoms are more difficult to manipulate than photons. Photon sources are more readily available and photon production rates are several orders of magnitude greater than is the case with atoms. Also, photon optics are better understood and more easily constructed. For example, consider the operation of splitting and recombining a wavefunction. A photon beamsplitter is conveniently made from glass, quartz, plastic, and other materials. An atom beamsplitter, in comparison, might be a finely-tuned laser pulse. Photons can also be deflected at arbitrary angles, while atom optics can only impart a limited amount of momentum, typically giving a few $\mathrm{cm} / \mathrm{s}$. These difficulties prevent the full theoretical advantage of atom interferometer from being realized. Nonetheless atom interferometry has proven useful for several applications, including measurements of fundamental constants 
[69], atom interaction studies [59], and gravitational sensing [52], in addition to inertial sensing.

Since the production of the first Bose-Einstein condensates more than 10 years ago, the intrinsic advantages of condensates over thermal atoms for atom interferometry have been recognized. The first advantage is slower atomic velocity. Condensates are essentially stationary, moving much slower than the hundreds of meters per second velocities typical of thermal atoms. Therefore little force is required to control the motion of a condensate atom, and arbitrary angular deflections are possible. In contrast, common deflection angles with thermal atoms are just a few $\mu \mathrm{rad}$. For example, the experiment of [28] used an optical standing wave beamsplitter which deflected the atoms by only $20 \mu \mathrm{rad}$. Consequently the arms of a condensate interferometer can be separated by a larger amount that in a thermal atom device, resulting in greater sensitivity. Between these extremes, atom interferometry can be implemented using laser cooled atoms, which typically move a few $\mathrm{cm} / \mathrm{s}$. This offers more control than in a thermal source, but still less than is possible with a condensate.

Condensates are also highly coherent. This can be understood by analogy to the coherence properties of optical lasers. The frequency bandwidth of a pulsed laser is approximately the reciprocal of the temporal width of the pulse,

$$
\Delta \nu \approx 1 / \Delta t
$$

The time scale $\Delta t$ can be referred to as the coherence time, which I shall now denote as 
$t_{c}$, and the coherence length $l_{c}$ is just the length of the pulse

$$
l_{c}=v t_{c}
$$

where the velocity is the speed of light. If an interferometer is used with path lengths that differ by more than $l_{c}$, the two pulses will not overlap on the detector and no interference will be observed. Less coherent light sources also have limited coherence times and lengths. For instance, light from a lamp has $l_{c} \sim \lambda$, the mean wavelength of the light. This severely constrains the acceptable path length difference in an interferometer.

Through analogy with an optical field, we see that for a condensate $v$ is the velocity of the atoms and $l_{c}$ is the length of the wavepacket, and for a thermal cloud $l_{c}$ is the atomic de Broglie wavelength $\lambda_{d B}$. The coherence length is the spatial distance over which the phase of the wavepacket can be reliably predicted [30]. Once again in order to view interference fringes, the path length difference must be less than $l_{c}$. Due to longer coherence lengths, condensates will exhibit high contrast interference fringes for larger path length differences than will thermal atoms.

Finally, condensate atoms are typically all in the same internal state. The two interferometric arms are created by separating the atoms into different motional states, which are later recombined into the same state. In contrast, atom interferometry experiments with warmer atoms must generally separate the two paths by transferring the atoms into a superposition of internal states [28]. The interferometer measurement is then made by detecting the number of atoms in each state. This causes the atoms to be sensitive 
to the phase of the field that drives the transitions between internal atomic states, and so this technique introduces decoherence and shortens the coherence time of the experiment. This type of manipulation is unnecessary with condensates since they are spatially well-localized, allowing direct observation of motional states with relative ease.

Though producing and working with condensates remains challenging, several groups have demonstrated Mach-Zehnder or Michelson interferometers using condensates [7,13, $26,29,57,60,65,66]$. However, all these experiments have been limited to measurement times of roughly ten milliseconds or less. We expect that the novel atom waveguide described here will permit significantly longer measurement times.

\subsection{Interferometer Design}

Our interferometer is subject to a number of design requirements. The most basic consideration is how the atoms will be transported through the device. Optical interferometers merely direct a light beam into the device and the photons freely propagate through at speed $c$. The path of the beam is altered using ordinary mirrors and beamsplitters. The atom interferometer must permit control of atomic trajectories, so that we can split the atomic cloud and send the wavepackets through the arms of the device. A standing wave laser field can be used as a diffraction grating to accomplish the splitting, with lasers or magnetic field gradients or gravity then used to propel the atoms through the interferom-

eter arms. This process is complicated by the fact that the atoms will undergo diffractive wavefunction expansion and repulsive atom interactions, causing an initially well-localized atomic wave packet to spread out. 
The simplest method is to orient the axis of the device vertically, allowing the two atomic wavepackets to fall freely under the influence of gravity $[7,26,65]$. This method is appealing since the problem of propagation is solved by simply dropping the atoms, without use of any additional fields that might induce dephasing. However, the measurement time is limited by the speed at which the condensate falls. For example, dropping the atoms a distance of $10 \mathrm{~cm}$ results in a $0.14 \mathrm{~s}$ measurement time.

The short measurement time is compounded by the fact that BEC experiments generically suffer from low production rates. This reduces the signal-to-noise ratio, since the statistical fluctuations in phase scale as $N^{-1 / 2}$ for number of atoms $N$. While thermal atomic beam experiments can produce $10^{9}$ atoms/s, condensate production rates are more typically $10^{5}$ atoms/s, with rates for laser-cooled uncondensed atoms falling in between. In order to make up for these low numbers, long interaction times will be required so that the overall phase is increased. This makes interferometers based on falling atoms unattractive, though some of the difficulties might be circumvented using either a fountain geometry [73] or a magnetic levitation approach [68].

The alternative possibility is to use trapped atoms. Condensate interferometers using atoms confined by either magnetic $[29,61,66]$ or optical $[13,57,60]$ fields have been demonstrated. Measurement times in these devices have been limited for a variety of reasons, but a common concern is the effect of interatomic interactions, which can introduce phase noise and cause spatial distortions in the cloud $[48,57,60]$.

We can estimate the effect of interactions using mean field theory. This is the primary mechanism of atomic interactions. The interaction energy of an atom in a condensate 
with density $n$ is

$$
U=\frac{4 \pi \hbar^{2} a}{m} n,
$$

where $m$ is the mass of the atom and $a$ is the s-wave scattering length which is $5.77 \mathrm{~nm}$ for ${ }^{87} \mathrm{Rb}$ [12]. The interaction energy leads to a phase shift

$$
\phi=\frac{U}{\hbar} t=\frac{4 \pi \hbar a}{m} n t .
$$

For a typical density of $n=10^{12} \mathrm{~cm}^{-3}$ and anticipated measurement time of $1 \mathrm{~s}$, the resultant interaction-induced phase shift is $53 \mathrm{rad}$. Ideally, both wavepackets would have the same number of atoms and their phase shifts would cancel out. In reality variations in spatial density will cause the phase shift to fluctuate, thus introducing noise [48]. This effect is known as phase distortion. The mean field energy also varies due to statistical fluctuations of the number of atoms $N$ in the condensate, with $\Delta N=N^{-1 / 2}$. This effect is known as phase diffusion. We can limit both effects by reducing the number of atoms in the condensate, at the cost of lower signal-to-noise. We can also then weaken the confining potential, so that the spatial density of the cloud decreases.

Confinement of the condensate in the interferometer also imposes geometrical constraints due to the need to avoid uncontrolled motional excitations [61,71]. To avoid all of these problems, one wants a trap capable of holding the atoms against gravity but otherwise as weakly confining as possible. Weak three-dimensional confinement has previously been observed [34], but we have developed a novel weakly confining waveguide that is particularly well-suited for the demands of atom interferometry. 
Our interferometer must also avoid all other sources of decoherence. Environmental noise induces phase shifts

$$
\Delta \phi_{\text {noise }}=\frac{\Delta E_{\text {noise }} t}{\hbar} .
$$

While we hope to exploit the sensitivity of the atoms to make improved sensors, it follows as well that atoms are also more sensitive to environmental noise than are photons. Furthermore, long interaction times cause condensate experiments to accumulate larger $\Delta \phi_{\text {noise }}$ than thermal atom experiments. An interferometer using trapped atoms to enhance interaction times must therefore take special care to shield out decoherence sources. Because we trap the atoms in a state with nonzero magnetic moment, one important noise source is ambient magnetic fields. Even a small magnetic field difference of $50 \mathrm{nG}$ between the two interferometer arms produces a phase shift of approximately $1 \mathrm{rad}$.

\subsubsection{Waveguide Structure and Fields}

Our waveguide is illustrated in Figure 4.2. It is based on a four-wire linear quadrupole and uses the time-orbiting potential (TOP) technique $[9,27,54]$. Four current-carrying rods provide a linear quadrupole field, with the zero line at the center. A rotating bias field pushes the zero away from the atoms to prevent Majorana losses. We preferred the TOP to other options because of its noise-reduction effects. As described above, our device will be sensitive to fluctuating ambient magnetic fields. The degree of shielding necessary to eliminate these fields would be challenging. Most of this noise arises from ac power lines and thermal drifts, so that the ambient fields tend to occur at low frequencies. A means of raising the sensitivity of our device to higher frequencies, where fewer noise sources are 


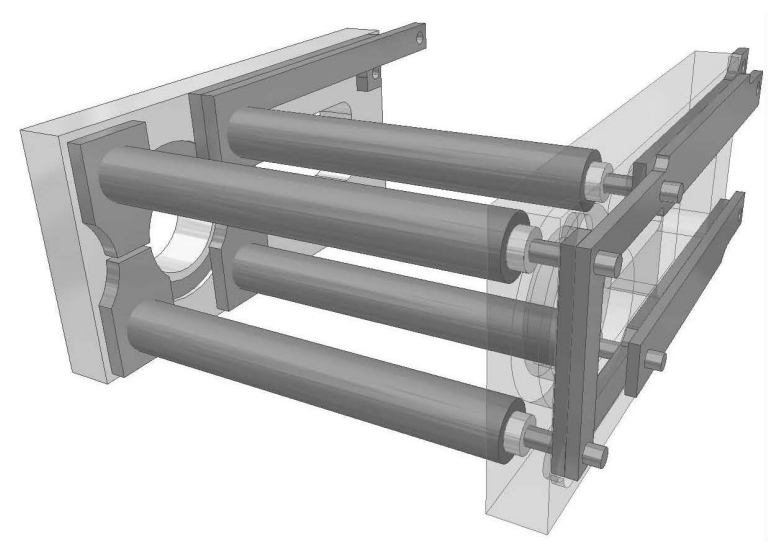

Figure 4.2: Scale drawing of the trap structure. The main fields are generated by the four horizontal rods, each of which is a coaxial pair. A pair consists of an outer conductor that is a 5-mm-diameter, 1-mm-wall oxygen-free high-conductivity copper tube, an alumina insulator, and an inner conductor that is a 1.6-mm-diameter copper wire. The rods are held by two boron nitride blocks, which also support the leads and circuit connections. The right block has been depicted as transparent in order to display the arrangement of the conductors. The rod centers form a square $15 \mathrm{~mm}$ on a side and the blocks are spaced $5 \mathrm{~cm}$ apart. The function of each of the conductors is described in Figure 4.5.

present, is therefore beneficial.

The rotating bias field of the TOP trap induces rotation of the atomic spins, as they adiabatically follow the field. Noise at frequencies below the rotation rate will tend to cancel out, so that the phase shift due to the noise has only a second order term

$$
\Delta \phi_{\text {field }}=\frac{\mu t}{2 \hbar} \frac{\delta B^{2}}{B_{0}} .
$$

Here $B_{0}$ is the strength of the rotating field, $t$ is the transit time and $\mu$ is the atomic magnetic moment. While the goal of lifting the pertinent frequencies to higher values is accomplished, we have also induced higher sensitivity to fluctuations at the rotation rate, since the noise then couples directly to the rotating spins.

We must select rotation rate judiciously to avoid phase noise. Our field must rotate 
more slowly than the Larmor precession rate,

$$
\omega_{L}=\frac{\mu_{B} B}{\hbar} \approx 10 \mathrm{MHz}
$$

so that the atomic spins are able to follow adiabatically. However, the atoms are trapped in a weak-field seeking state. In order to prevent them from moving towards the zero, we must rotate more rapidly than the frequency of the atomic motion, at about $10 \mathrm{~Hz}$. A range of $1-100 \mathrm{kHz}$ is appropriate. To narrow this range, we analyzed the noise spectrum of our laboratory. We used two small pickup coils to sense the ambient B field, by detecting the field gradient between them. The coils were aligned oppositely, so that in the presence of a uniform field the signals from the two coils would cancel each other out. We calibrated each coil separately, by measuring the known field induced from a third coil. This measurement was frequency dependent. We scanned a range of frequencies for calibration, and then recorded the ambient noise of the surrounding environment over the same range. The resulting noise spectrum for the laboratory environment is displayed in Figure 4.3. The noise floor is lowest around $10 \mathrm{kHz}$, but we also want to avoid discrete peaks. We selected $11.88 \mathrm{kHz}$ rotation rate because this is a multiple of $60 \mathrm{~Hz}$ and its first few harmonics, which in principle allows for perfect noise cancellation.

While rotating the bias field works well to limit the effects of ambient noise sources, it will not address the mean field interactions. I mentioned earlier that phase distortion can be limited by using a weak confinement field to lower the spatial density $n$, but conventional TOP traps are not especially weak. The obvious way to reduce the confinement 


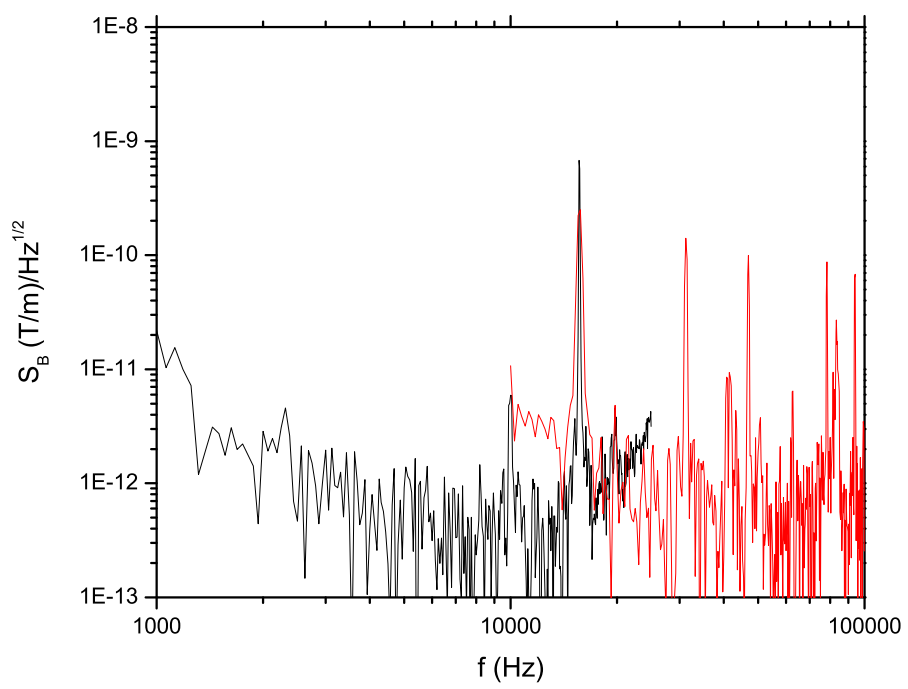

Figure 4.3: Noise spectrum.

strength is to reduce the magnetic field amplitude, but we cannot lower the force below what is required to counter gravity otherwise the atoms will fall out of the trap. The solution we have found is to oscillate not only the bias field, but also the quadrupole field. With the appropriate choice of phase, this causes the field zero to oscillate back and forth above the atoms. The atoms are constantly attracted to the overhead zero, and we can weaken the confinement further than would otherwise be possible. Figure 4.4 gives a simple illustration of the trajectory of the B field zero in our trap.

To understand this mechanism, suppose the oscillating quadrupole field is

$$
\mathbf{B}_{Q}=B_{Q}^{\prime}(x \hat{\mathbf{x}}-z \hat{\mathbf{z}}) \cos \Omega t
$$

where $\hat{\mathbf{x}}$ and $\hat{\mathbf{z}}$ are the transverse directions and $\hat{\mathbf{y}}$ is along the axis of the waveguide. The 


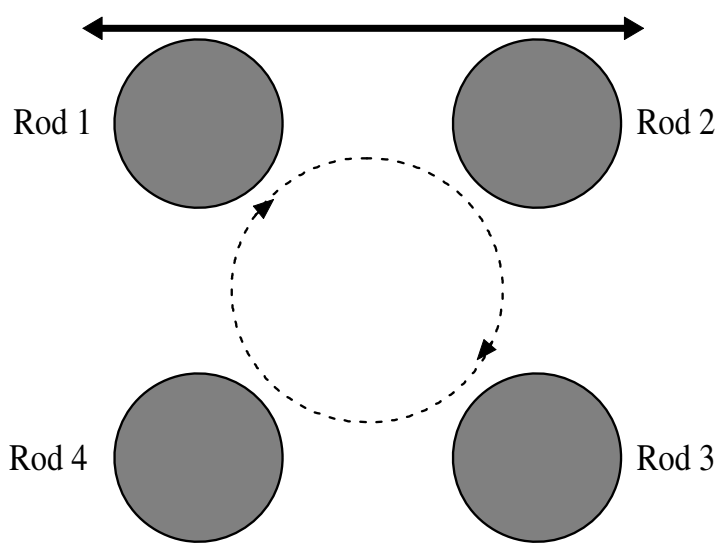

Figure 4.4: Illustration of rotating field. The waveguide structure is viewed from one end, so that the 4 rods appear as circles. The dotted circle indicates the path of the B field zero when only the bias field is rotated. The solid line above the rods indicates the path traced by the zero when the confinement quadrupole field is oscillated along with the bias field.

$\hat{\mathbf{z}}$ direction is vertical. The bias field is

$$
\mathbf{B}_{0}=B_{0}(\hat{\mathbf{x}} \sin \Omega t+\hat{\mathbf{z}} \cos \Omega t)
$$

with rotation frequency $\Omega=11.88 \mathrm{kHz}$. We can solve for the the time-averaged field magnitude,

$$
\langle|\mathbf{B}|\rangle=\left\langle\left|\mathbf{B}_{Q}+\mathbf{B}_{0}\right|\right\rangle
$$

just as in Section 3.3.1. In the first step the vector components of the quadrupole and bias fields are added together, and then squared. The result is

$$
|\mathbf{B}|^{2}=B_{0}^{2}+B_{Q}^{\prime 2}\left(x^{2}+z^{2}\right) \cos ^{2} \Omega t+B_{Q}^{\prime} B_{0}\left(\cos \Omega t \sin \Omega t-\cos ^{2} \Omega t\right) .
$$

In order to take the square root, we again use the binomial expansion, preserving only 
terms up to order two, so that

$$
\begin{aligned}
|\mathbf{B}|=B_{0}+\frac{B_{Q}^{\prime 2}}{2 b_{0}}\left(x^{2}+z^{2}\right) & \cos ^{2} \Omega t-B_{Q}^{\prime} z \cos ^{2} \Omega t \\
& -\frac{1}{8} \frac{B_{Q}^{\prime 2}}{B_{0}}\left(x^{2} \cos ^{2} \Omega t \sin ^{2} \Omega t+z^{2} \cos ^{4} \Omega t\right)
\end{aligned}
$$

Finally we average over the oscillating terms. The resulting time-averaged field magnitude, to second order in the coordinates, is

$$
\langle|\mathbf{B}|\rangle=B_{0}-\frac{1}{2} B_{Q}^{\prime} z+\frac{B_{Q}^{\prime 2}}{16 B_{0}}\left(3 x^{2}+z^{2}\right) .
$$

The total potential energy from this field is

$$
U=\mu B+m g z=\mu B_{0}-\frac{1}{2} \mu B_{Q}^{\prime} z+m g z+\frac{1}{2} m\left(\omega_{x}^{2} x^{2}+\omega_{z}^{2} z^{2}\right)
$$

for atomic mass $m$, gravitational acceleration $g$, and magnetic moment $\mu$. We set the gradient $B_{Q}^{\prime}=2 m g / \mu$ to support the atoms against gravity and obtain trap frequencies

$$
\omega_{x}=\left(\frac{3 m g^{2}}{2 \mu B_{0}}\right)^{\frac{1}{2}} \quad \text { and } \quad \omega_{z}=\frac{\omega_{x}}{\sqrt{3}} .
$$

For a $10 \mathrm{G}$ bias field, this gives trap frequencies $\omega_{x}=2 \pi \times 7 \mathrm{~Hz}$ and $\omega_{z}=2 \pi \times 4 \mathrm{~Hz}$ for $\mathrm{Rb}$ atoms in the $|22\rangle$ state. 

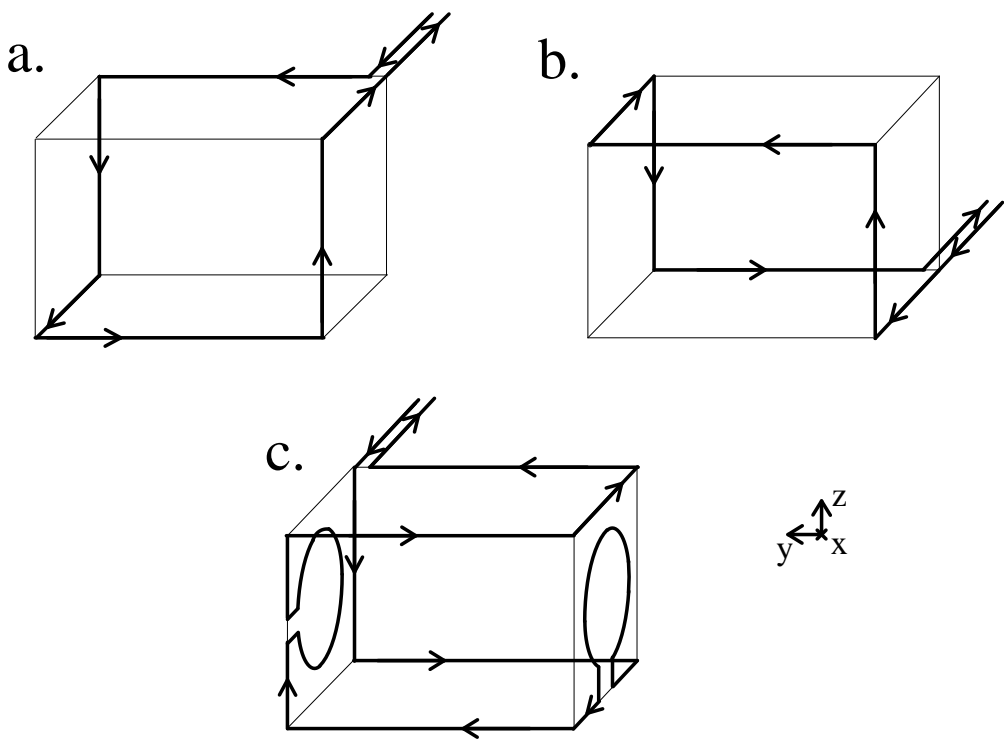

Figure 4.5: Current flow through waveguide, indicated by thickened lines with directional arrows. The four rods in Figure 4.2 are depicted here as the edges of a rectangular box. Circuits (a) and (b) refer to the current through the inner conductors, which provides the oscillating bias field. Circuit (c) is composed of the outer conductors, which supply the confinement quadrupole field. The end loops on circuit (c) help to minimize the axial quadrupole field.

\subsection{Operation}

The waveguide is made of machined copper rods held inside a vacuum chamber, as shown in Figure. 4.2. Each of the four rods is a coaxial pair. As Figure 4.5 illustrates, the four outer conductors are connected in one circuit that provides the quadrupole field while the inner conductors form two circuits used to generate the two components of the bias field. The end loops shown on the quadrupole circuit help minimize the axial quadrupole field.

The trap is mounted on two boron nitride blocks. As shown in Figure 4.6, the trap is then attached to several copper blocks that deliver current and remove heat. The first drawing in the figure is viewed from the side of the trap, looking along the axis of the rods. The second drawing is viewed from above. 

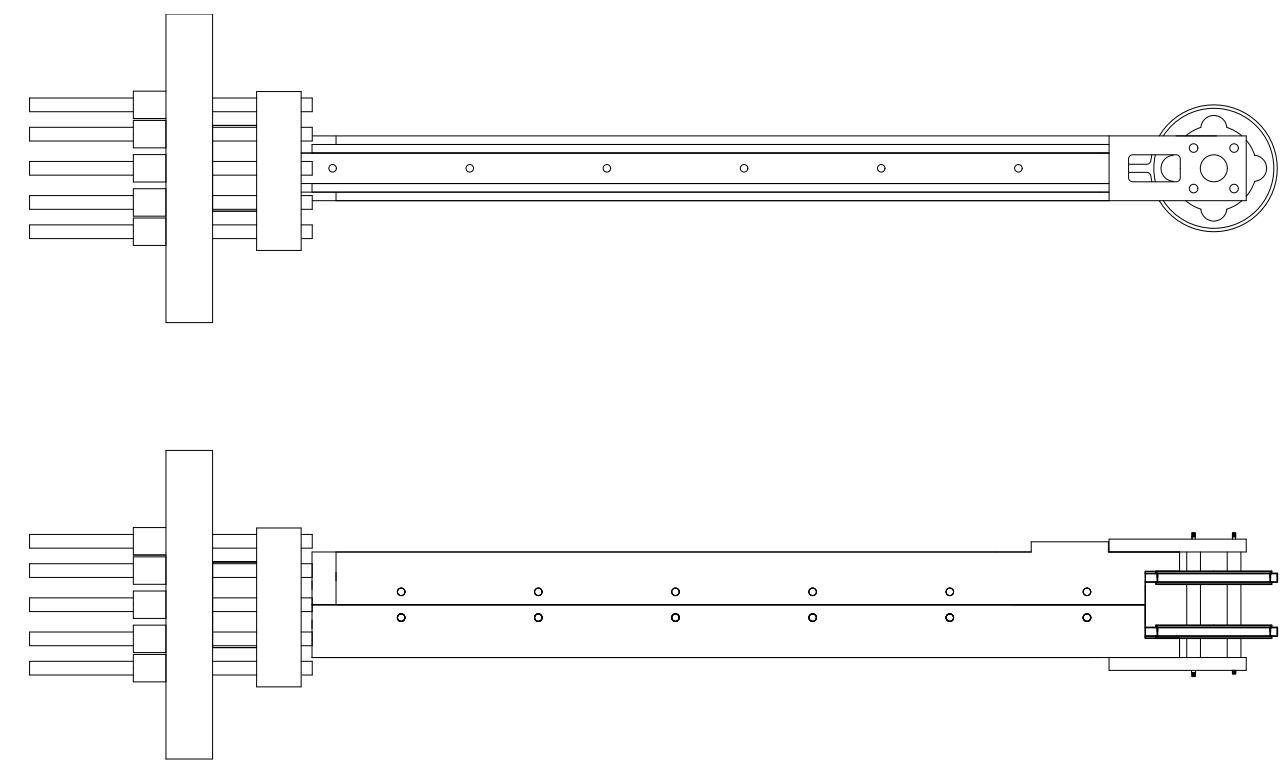

Figure 4.6: Outline of entire assembled waveguide structure. The first drawing is viewed from the side, looking along the axis of the rods. The circular shape on the end of the mount is an axial endloop, included to provide the option of an axial confinement field. The second drawing is viewed from above. Here the endloops appear as horizontal lines and the rods are drawn vertically.

The leads of the circuits do have an appreciable effect on the trap potential. For instance, any residual axial component causes the quadrupole field to become

$$
\mathbf{B}_{Q}=(a x \hat{\mathbf{x}}+c y \hat{\mathbf{y}}-b z \hat{\mathbf{z}}) \cos \Omega t
$$

with $c=b-a$. A more accurate description of the potential requires the inclusion of many other first- and second-order terms in the magnetic fields. Categorizing all such terms is prohibitive, but we have found that in the relevant parameter range, the total 
average field magnitude is well approximated by

$$
\begin{aligned}
\langle|\mathbf{B}|\rangle=B_{0}-\frac{1}{2} b z & +\left(\frac{3}{16 B_{0}} a^{2}+\alpha B_{0}\right) x^{2} \\
& +\left(\frac{1}{4 B_{0}} c^{2}+\gamma B_{0}\right) y^{2}+\left(\frac{1}{16 B_{0}} b^{2}+\beta B_{0}\right) z^{2}
\end{aligned}
$$

which yields frequencies

$$
\begin{aligned}
& \omega_{x}=\left[\frac{2 \mu}{m}\left(\frac{3}{16 B_{0}} a^{2}+\alpha B_{0}\right)\right]^{\frac{1}{2}} \\
& \omega_{y}=\left[\frac{2 \mu}{m}\left(\frac{1}{4 B_{0}} c^{2}+\gamma B_{0}\right)\right]^{\frac{1}{2}} \\
& \omega_{z}=\left[\frac{2 \mu}{m}\left(\frac{1}{16 B_{0}} b^{2}+\beta B_{0}\right)\right]^{\frac{1}{2}} .
\end{aligned}
$$

Here $a, b$ and $c$ are from Eq. (4.20) and $\alpha, \beta$, and $\gamma$ come from variations in the bias fields. We obtained this form by modeling the total field using the Biot-Savart law and the mechanical design of the leads. For example, consider the line segment shown in Figure 4.7. Here $d l$ is an element of length along the wire, $r_{1}$ and $r_{2}$ are the ends of the line segment, $\mathbf{x}$ is the vector from the source element to the measurement point $(x, y, z)$, and $\mathbf{r}$ is the vector from the origin to the measurement point. For a current $I$ flowing through the wire, the total field from the wire segment is

$$
\mathbf{B}=\frac{\mu_{0} I}{4 \pi} \int \frac{d \mathbf{l} \times \mathbf{x}}{x^{2}} .
$$




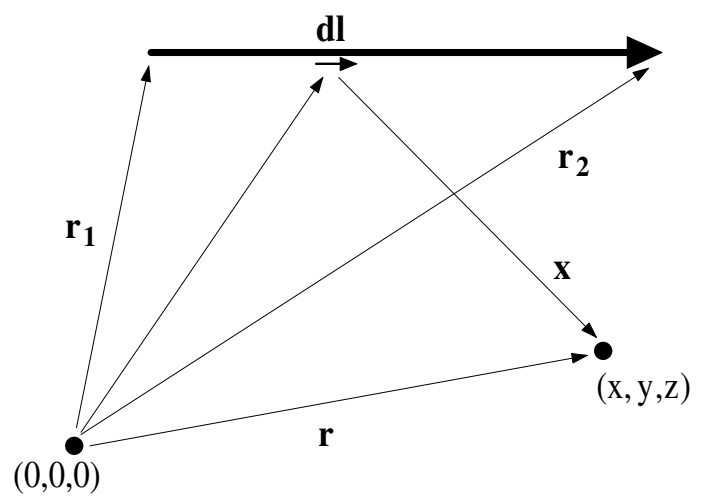

Figure 4.7: Description of coordinate system for calculation of B field from a segment of current-carrying wire.

Let the length of the wire segment be $\mathbf{L}=\mathbf{r}_{2}-\mathbf{r}_{1}$ and let $\mathbf{a}=\mathbf{r}-\mathbf{r}_{1}$. Then

$$
\mathbf{B}=\frac{\mu_{0} I}{4 \pi} \frac{\mathbf{L} \times \mathbf{a}}{|\mathbf{L} \times \mathbf{a}|^{2}}\left[\frac{\mathbf{L} \cdot(\mathbf{L}-\mathbf{a})}{|\mathbf{L}-\mathbf{a}|}+\frac{\mathbf{L} \cdot \mathbf{a}}{|\mathbf{a}|}\right]
$$

Our model performs this calculation for each segment of wire in the trap, including the circular loops, and sums up all contributions to find the net field at any point in space for a specified current.

This model is simple in that it ignores the thickness of the rods and leads, instead approximating each wire segment as a line at the center of the wire. The model predicts $B_{0} / I_{0}=0.40 \mathrm{G} / \mathrm{A}, a / I_{Q}=-0.83 \mathrm{G} /(\mathrm{A} \mathrm{cm}), b / I_{Q}=-0.86 \mathrm{G} /(\mathrm{A} \mathrm{cm}), \alpha=-0.11 \mathrm{~cm}^{-2}$, $\beta=-0.061 \mathrm{~cm}^{-2}$, and $\gamma=0.019 \mathrm{~cm}^{-2}$, where $I_{0}$ is the bias current amplitude and the $I_{Q}$ is the quadrupole current amplitude. These values were coarsely verified using a gaussmeter, yielding $B_{0} / I_{0} \approx 0.4 \mathrm{G} / \mathrm{A}$ and $a / I_{Q} \approx b / I_{Q} \approx 0.8 \mathrm{G} /(\mathrm{A} \mathrm{cm})$.

The three trap circuits have similar impedances, presenting a $10 \mathrm{~m} \Omega$ resistive and $0.3 \mu \mathrm{H}$ inductive load. The circuits are driven with an actively stabilized commercial 
audio amplifier, using transformers to match the amplifier's output impedance. We chose the resistance of the load to set the power dissipation. The inductance sets the required voltage, with $\omega L>R$ here. The trap is mounted on several copper blocks that deliver the current and remove heat. The measured thermal coefficient of the trap structure is $2 \mathrm{~W} / \mathrm{K}$. The quadrupole field requires a current of $38 \mathrm{~A}$ to cancel gravity and a bias field of $20 \mathrm{G}$ requires $I_{0}=50 \mathrm{~A}$ in both bias circuits. The total power to be dissipated is $P=\frac{1}{2} I^{2} R=32 \mathrm{~W}$, yielding a total temperature rise of about $16 \mathrm{~K}$.

The operation of our BEC system is described in Chapter 3. The TOP trap used during evaporative cooling is produced by the quadrupole field of the magnetic trap and the bias field of the waveguide. Therefore we perform evaporative cooling while the atoms are located within the waveguide structure, although the waveguide's linear quadrupole field remains off until after the condensates have been created.

We used evaporative cooling to obtain a more accurate calibration of the bias field. For a bias field driven by a control voltage of $2 \mathrm{~V}$, we determined that all atoms were driven out of the trap when the RF field was scanned down to $2.585 \mathrm{MHz}$. Using the relation

$$
\frac{1}{2} \mu B_{0}=h \nu_{\text {stop }},
$$

we solved for a bias field of $B_{0}=3.69 \mathrm{G}$. Similarly, we found bias fields of $9.97 \mathrm{G}$ and $16.28 \mathrm{G}$ for control voltages of $5 \mathrm{~V}$ and $8 \mathrm{~V}$. Letting $B_{0}=\kappa V$, we applied a linear fit to our three data points and determined that $\kappa=2.1 \mathrm{G} / \mathrm{V}_{\text {control }}$. If we define the relation between the field and the current as $B_{0}=\gamma I$, then to calibrate the field we will solve for $\gamma$. The current through each pair of conductors can be written as $I_{1}=A \cos \omega t$ and $I_{2}=A \sin \omega t$, 
where $A$ is the current amplitude. At $t=0$ the peak-to-peak current through the system is $I_{p p}=2 A$, so that $\gamma=2 B_{0} / I_{p p}$. We monitored the output of the function generators used to make the bias field, and found that $V_{\text {monitor }}=9.55 \mathrm{~V}_{p p}$ at $10 \mathrm{~V}_{\text {control }}$. Since the current is $10 \mathrm{~A} / \mathrm{V}$ times $\mathrm{V}_{\text {monitor }}$, we find that the peak-to-peak current is $I_{p p}=9.55 \mathrm{~A}_{p p} / \mathrm{V}_{\text {monitor }}$. Combining all of this data, the bias field can be calibrated according to

$$
\gamma=\frac{2 B_{0}}{I_{p p}}=\frac{2 \kappa V_{c}}{9.55 \mathrm{~A} / \mathrm{V} \times V_{c}}=0.440 \mathrm{G} / \mathrm{A}
$$

The actual bias field then is $0.440 \mathrm{G} / \mathrm{A}$ times the current amplitude $I_{0}$.

Once the condensate is made the waveguide quadrupole field is ramped on and the spherical quadrupole field ramped off. The centers of the main trap and the waveguide do not exactly coincide, so the fields are ramped with a $7 \mathrm{~s}$ time period to enable the atoms to move adiabatically to the new local B field minimum. The details of the ramp are shown in Appendix F, which contains the ADwin pulse sequence used to load the condensate into the waveguide. We do not observe any losses in the transfer. Figure 4.8 shows snapshots of the cloud as the atoms are being transferred.

We selected the strength of the quadrupole confinement field by applying the field necessary to keep the atoms in the same vertical position as the waveguide was loaded. If we did not supply a large enough gradient, the cloud assumed a slightly lower position and the image of the cloud was out of focus. We found that when the trap was loaded with bias field set to $B_{0}=3.69 \mathrm{G}$, we required that the quadrupole field control voltage $V_{Q}$ must be $7.8 \mathrm{~V}$. Similarly, we found $V_{Q}=8.0 \mathrm{~V}$ for $B_{0}=9.97 \mathrm{G}$ and $V_{Q}=8.2 \mathrm{~V}$ for 


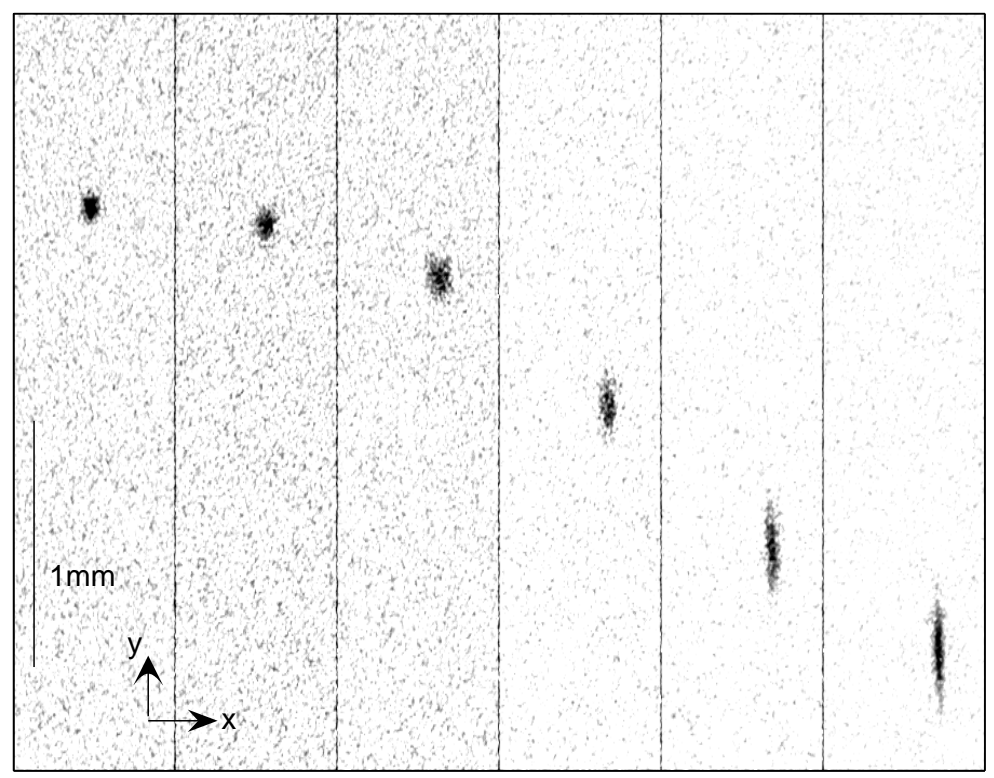

Figure 4.8: Loading a Bose-Einstein condensate into the waveguide. The sequence of pictures show the trapped condensate as the static quadrupole field is gradually turned off: (a) $29 \mathrm{G} / \mathrm{cm}$ (b) $19 \mathrm{G} / \mathrm{cm}$ (c) $9.7 \mathrm{G} / \mathrm{cm}$ (d) $3.9 \mathrm{G} / \mathrm{cm}$ (e) $1.9 \mathrm{G} / \mathrm{cm}$ (f) $0 \mathrm{G} / \mathrm{cm}$. During the loading, the cloud moves because the centers of the external quadrupole and the waveguide are not coincident. The bias field here is $3.69 \mathrm{G}$, and the final trap frequencies are $\omega_{x}=2 \pi \times 11 \mathrm{~Hz}, \omega_{z}=2 \pi \times 6.2 \mathrm{~Hz}$, and $\omega_{y}=2 \pi \times 0.6 \mathrm{~Hz}$.

$B_{0}=16.28 \mathrm{G}$.

As Figure 4.8 demonstrates, the atomic cloud undergoes two noticeable changes as it is transferred to the waveguide. First, the cloud changes location by about $2 \mathrm{~mm}$ along the waveguide axis. We believe this is because the center of the main quadrupole trap does not exactly coincide with the center of the trap formed by the waveguide. We can control one degree of freedom by adjusting the distance the trap moves when transferring the atoms from the MOT chamber to the science cell. We found that $543 \mathrm{~mm}$ was the correct distance to center the atoms in the waveguide along the $x$ direction. However, to adjust the $y$ direction we would have to physically move the translation stage closer to or further 
from the science cell. We elected to slowly ramp down the main quadrupole field and slowly ramp on the waveguide quadrupole field to allow the atoms to adiabatically follow the location of the center of the trap. (Note, since this writing residual oscillations of cloud have been observed even with the slow ramp, so it is possible that this displacement of the cloud is part of those oscillations.)

We also see that the cloud stretches out along the $y$ axis. This is due to the relatively weak confinement along the axis of the guide. If not for the residual axial confinement, the cloud would be unconfined in the $y$ direction and spread without limit.

To solve for the values of the parameters of Eq. (4.22), it was necessary to characterize the potential of the atom waveguide. After loading atoms into the trap, we perturbed the cloud in an effort to induce oscillations at the trap frequencies. For the $x$ and $y$ oscillations, we altered the Compumotor system to drive the coils a few $\mathrm{mm}$ short of the center of the waveguide. We then evaporated the cloud to just above the BEC transition, so that our oscillation measurements would avoid the effects of atomic interactions within the condensate. The transfer of the cold cloud to the waveguide was initiated as before, by ramping the magnetic fields. However, instead of finishing the ramp, with $0.5 \mathrm{~V}$ to go we suddenly switched the external quadrupole field to $0 \mathrm{~V}$. This caused the atoms to experience a jolt as they moved into the waveguide, and set up oscillations of the cloud at the trap frequencies.

We observed both center-of-mass oscillations, where the entire cloud sloshed back and forth within the trap, and breathing mode oscillations, where the width of the cloud periodically expanded and contracted. For breathing modes, the oscillation frequency is 

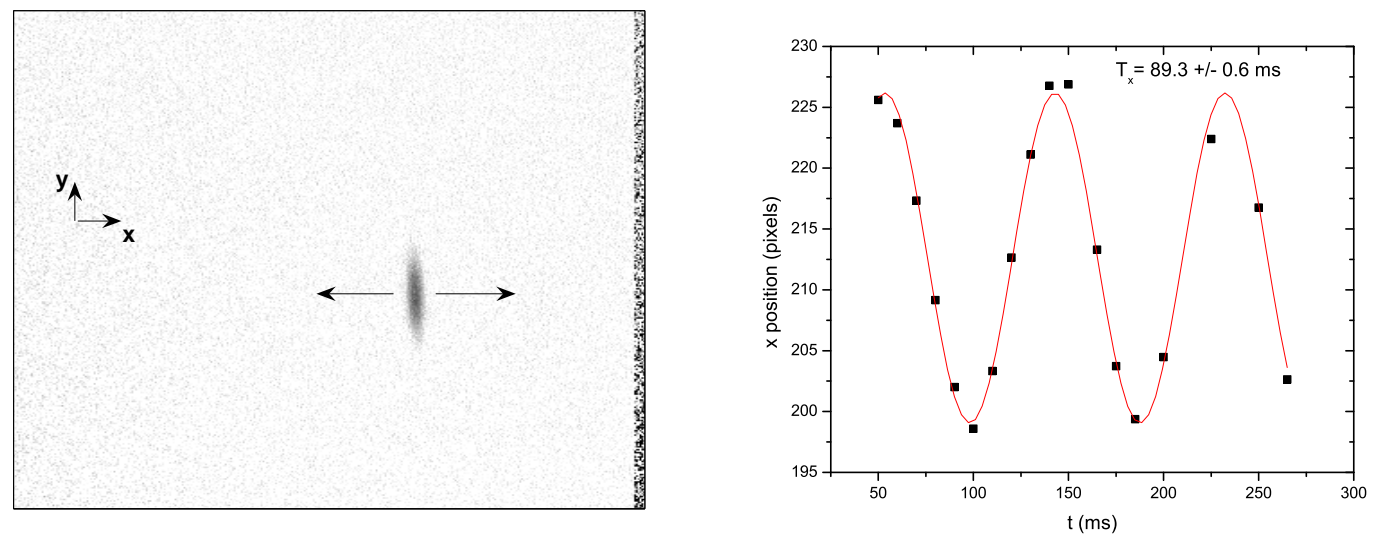

Figure 4.9: Top view of oscillating condensate. We measure the trap frequency in the $x$ direction by observing center-of-mass oscillations of the condensate. The graph to the right is a scatter plot of cloud position vs. time, with a damped sine wave fit. The fit indicates a period of $89.4 \mathrm{~ms}$, for a trap frequency of $\omega_{x}=2 \pi \times 11.2 \mathrm{~Hz}$. Here the bias field is $3.69 \mathrm{G}$, with waveguide control voltages $V_{\text {bias }}=1.8$ and $V_{\text {Quad }}=7.8$.

actually twice the trap frequency. As it turned out, we readily observed center-of-mass oscillations in the $x$ direction and breathing mode oscillations in the $y$ direction, so we recorded that data. Our $x$ and $y$ data for $B_{0}=3.69 \mathrm{G}$ are displayed in Figures 4.9 and 4.10. These tests were done at temperatures of about $1 \mu \mathrm{K}$, which is above the BEC transition. From the periods we determined the trap frequencies as a function of the applied currents.

Our imaging system was constructed so as to view the condensate from above, so that our images of the cloud are in the $x y$ plane. Therefore $z$ oscillations were not directly observable. We arranged one of our infrared security cameras to peer into the chamber from the side. This second camera did not have the resolution or magnification of the Apogee AP47 used for the bulk of the condensate images. We found that we could obtain clear pictures of the cloud with this camera by evaporating only to about $2 \mu \mathrm{K}$, so that we 

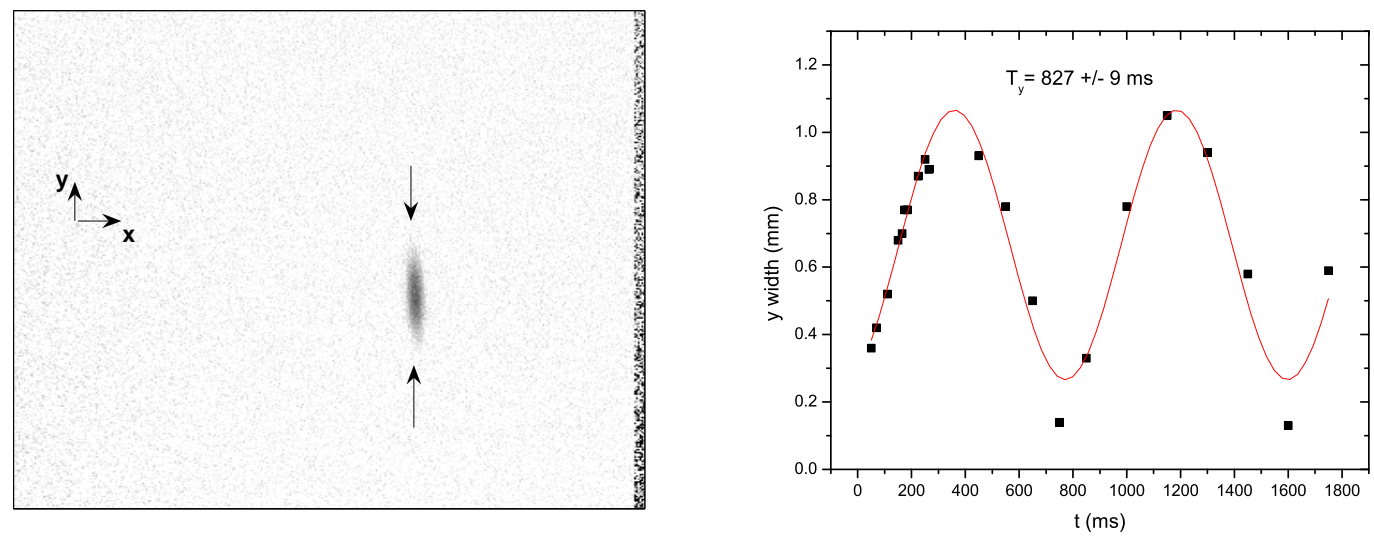

Figure 4.10: Top view of oscillating condensate. We measure the trap frequency in the $y$ direction by observing breathing mode oscillations of the cloud. The graph to the right is a scatter plot of the length of the cloud in the $y$ direction vs. time, with a damped sine wave fit. The fit indicates a period of $825 \mathrm{~ms}$. Because this is a breathing mode, we must multiply the period by 2 . We then find a trap frequency of $\omega_{y}=2 \pi \times 0.6 \mathrm{~Hz}$. Here the bias field is $3.69 \mathrm{G}$, with waveguide control voltages $V_{\text {bias }}=1.8$ and $V_{\text {Quad }}=7.8$.

imaged $z$ oscillations of a slightly hotter cloud than in the case of the $x$ and $y$ oscillations.

We observed center of mass motion of the cloud in the $z$ direction, as shown in Figure 4.11.

\begin{tabular}{||c|c|c|c|c|c||}
\hline $\mathrm{V}_{Q}(\mathrm{~V})$ & $\mathrm{V}_{\text {bias }}(\mathrm{V})$ & $\mathrm{B}_{0}(\mathrm{G})$ & $\nu_{x}(\mathrm{~Hz})$ & $\nu_{z}(\mathrm{~Hz})$ & $\nu_{y}(\mathrm{~Hz})$ \\
\hline 7.8 & 1.8 & 3.69 & 11.2 & 6.17 & 0.61 \\
8.0 & 4.8 & 9.97 & 7.29 & 4.05 & 0.88 \\
8.2 & 9.8 & 16.28 & 6.27 & 3.5 & 1.09 \\
\hline
\end{tabular}

Table 4.1: Measured trap frequencies for three bias field strengths.

We measured the frequencies over a range of bias fields from 3 to $16 \mathrm{G}$, obtaining the data in Table 4.1. From this data, we solved for the trap parameters in our model Eq. (4.22). We used a multivariable mimimization routine, with $a, b, c, \alpha, \beta$, and $\gamma$ allowed to vary as each frequency was calculated. We fit to the measured frequencies at each bias 

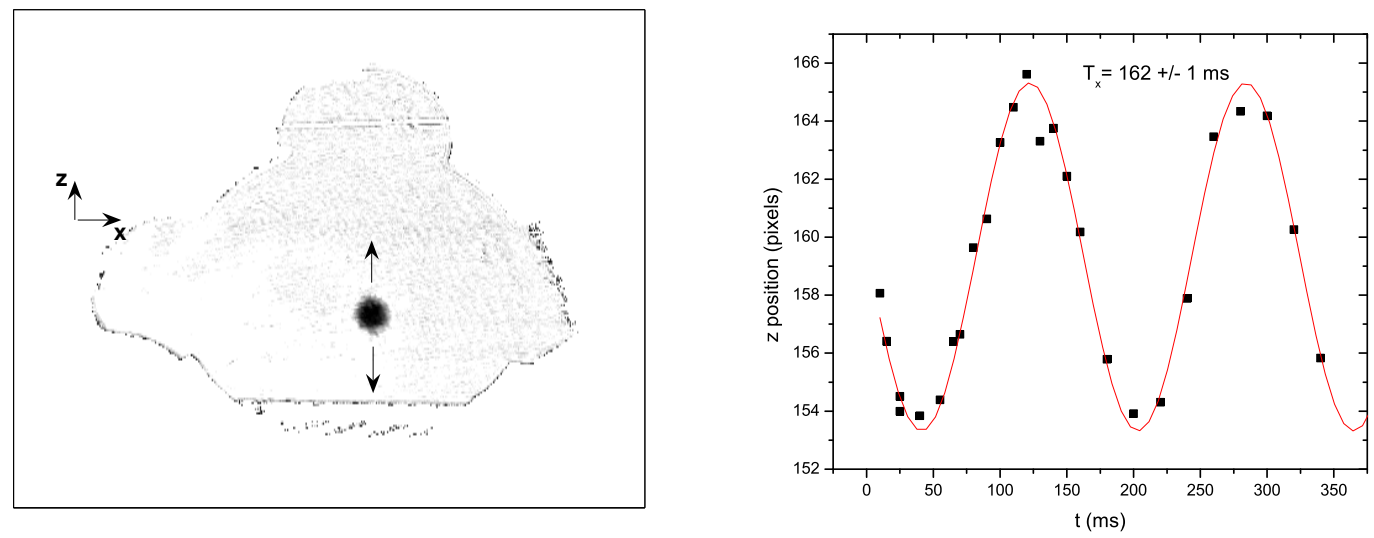

Figure 4.11: Side view of oscillating condensate. We measure the trap frequency in the $z$ direction by observing center-of-mass oscillations of the cloud. The graph to the right is a scatter plot of cloud position vs. time, with a damped sine wave fit. The fit indicates a period of $162 \mathrm{~ms}$, for a trap frequency of $\omega_{z}=6.17 \mathrm{~Hz}$. Here the bias field is $3.69 \mathrm{G}$, with waveguide control voltages $V_{\text {bias }}=1.8$ and $V_{\text {Quad }}=7.8$.

field strength, and found the solutions with the minimum $\chi^{2}$. In this way we found $|a| / I_{Q}=0.734 \mathrm{G} /(\mathrm{A} \mathrm{cm}),|b| / I_{Q}=0.709 \mathrm{G} /(\mathrm{A} \mathrm{cm}), \alpha=0.17 \mathrm{~cm}^{-2}, \beta=0.05 \mathrm{~cm}^{-2}$, and $\gamma=0.02 \mathrm{~cm}^{-2}$. The quadratic coefficients are rather different from our model predictions, though the order of magnitude is correct. We do not have a good explanation for this. Perhaps the cloud is not at the center of the waveguide structure, or other external gradients are present. Using the empirical coefficients, Eq. (4.22) reproduces the measured frequencies to an accuracy of about $0.1 \mathrm{~Hz}$ over the range of bias fields tested. Figure 4.12 compares the measured and predicted trap frequencies.

The weakest confinement can be implemented at $B_{0}=20.5 \mathrm{G}$, with trap frequencies $\omega_{x}=2 \pi \times 6.0 \mathrm{~Hz}, \omega_{y}=2 \pi \times 1.2 \mathrm{~Hz}$ and $\omega_{z}=2 \pi \times 3.3 \mathrm{~Hz}$. We did not measure these frequencies, but solved for them using Eq. (4.22) and the empirically-determined coefficients. Besides being useful for minimizing interatomic interactions, this weak con- 


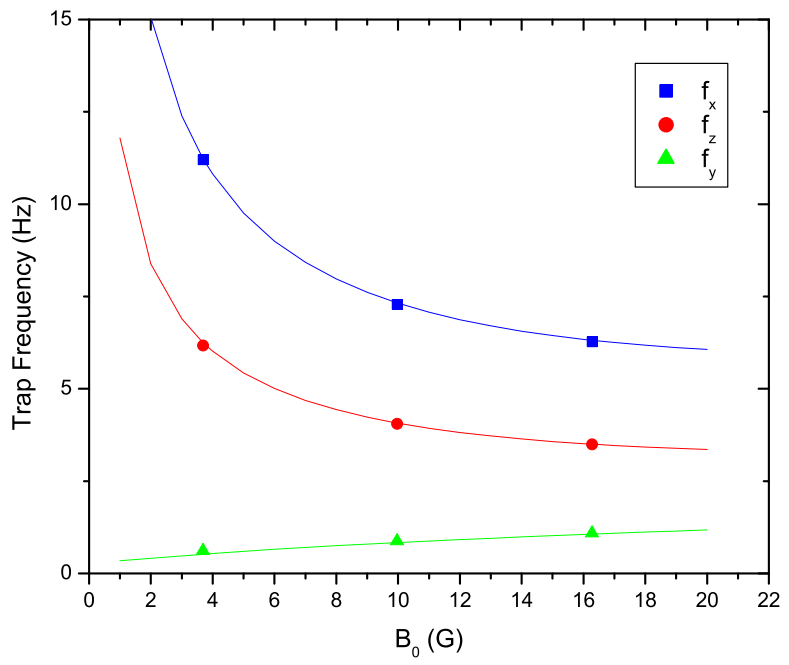

Figure 4.12: Comparison of measured and predicted trap frequencies. The frequencies predicted with our multivariable fit agree with the measured trap frequencies to within $0.1 \mathrm{~Hz}$. The squares, circles and triangles are the measured frequencies in the $x, z$ and $y$ directions. The lines are generated by Eq. (4.22) and the measured values of the parameters $a, b, c, \alpha, \beta$ and $\gamma$.

finement also allows the atoms to be adiabatically expanded to very low temperatures.

Figure 4.13 shows an image of $1.6 \times 10^{3}$ atoms in the trap with this bias field. The critical temperature $T_{c}$ is

$$
T_{c}=0.94 \frac{\hbar \omega_{0}}{k_{B}} N^{1 / 3}
$$

where $\omega_{0}=\left(\omega_{x} \omega_{y} \omega_{z}\right)^{1 / 3}$. To find the temperature of the cloud in the trap, we use

$$
N_{0}=N\left[1-\left(\frac{T}{T_{c}}\right)^{3}\right]
$$

where $N_{0} / N$ is the condensate fraction which is 0.76 in this instance. Rearranging,

$$
T=T_{c}\left(1-\frac{N_{0}}{N}\right)^{1 / 3}=0.62 T_{c} .
$$


We find that $T_{c}=1.35 \mathrm{nK}$ and $T=850 \mathrm{pK}$. Although lower temperatures have been observed in $\mathrm{Na}$ [34], this is the lowest temperature achieved for Rb atoms of which we are aware.

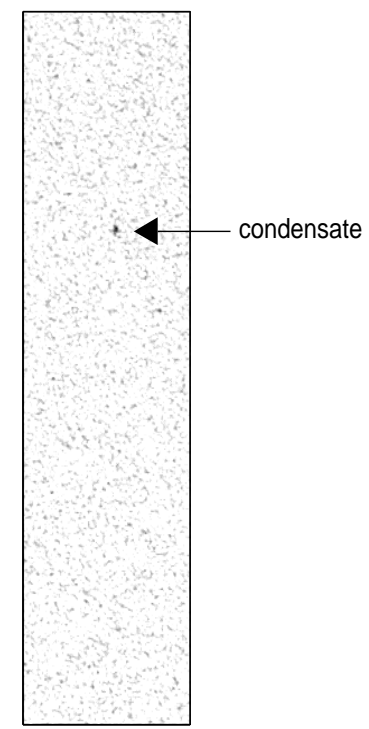

Figure 4.13: An atomic cloud after increasing the bias field to $20.5 \mathrm{G}$, with estimated trap frequencies $\omega_{x}=2 \pi \times 6.0 \mathrm{~Hz}, \omega_{y}=2 \pi \times 1.2 \mathrm{~Hz}$ and $\omega_{z}=2 \pi \times 3.3 \mathrm{~Hz}$. We estimate the temperature of this cloud to be $850 \mathrm{pK}$.

\subsection{Interferometer Measurement}

With the successful demonstration of our trap, we are now preparing to explore condensate interferometry. In order to observe high contrast interference fringes, we require that the source condensate be split into two wavepackets with narrow momentum distributions. For optimum spatial resolution between the two interferometer arms, these wavepackets must also have large momentum separations [72]. We plan to conduct experiments similar 
to those of Wang et al., [66], using a Bragg laser pulse to split and recombine condensate wave packets.

The process of splitting and recombining the wavepackets is described in detail in [66] and [72], so I will give only a brief overview of the process. Our condensate interacts with a far-off resonant standing wave laser pulse, such as created by using a mirror to reflect the beam back onto itself. The wavefunction of the condensate evolves in time as $\Psi \rightarrow \Psi e^{i E t / \hbar}$. The standing wave laser pulse has intensity

$$
I=I_{0} \cos ^{2} k z .
$$

The light shifts the energy levels of an atom as $E \propto I$, so that

$$
\frac{E t}{\hbar}=\beta \cos ^{2} k z=\frac{1}{2} \beta(1+2 \cos 2 k z) \approx \beta \cos 2 k z,
$$

where we have discarded the 1 because it results in an overall phase $e^{i \beta}$ which is uniform and can thus be ignored. If $\beta$ is small, we can write the wavefunction as

$$
\begin{aligned}
\Psi & \rightarrow \Psi e^{i \beta \cos 2 k z} \\
& =\Psi(1+i \beta \cos 2 k z) \\
& =\Psi\left[1+i \frac{\beta}{2}\left(e^{i 2 k z}+e^{-i 2 k z}\right)\right] \\
& \equiv \Psi_{0}+i \frac{\beta}{2}\left(\Psi_{+}+\Psi_{-}\right) .
\end{aligned}
$$

Here the wavefunction has been split into three separate pieces, where $\Psi_{0}$ represents 
the original wavefunction with zero momentum and $\Psi_{+}$and $\Psi_{-}$are wavepackets with momentum $\pm 2 \hbar k$. These packets fly apart, and will eventually be brought back together to interfere. This establishes the basic idea of how the splitting works. It is limited, however, to $\beta \ll 1$, which means only a small fraction of the atoms are used.

To obtain larger $\beta$ we can write the exponential as a Bessel function.

$$
e^{i \beta \cos 2 k z}=\sum_{n} J_{n}(\beta) e^{i 2 n k z}
$$

Then the initial wavefunction

$$
\Psi(0)=\sum_{n} J_{n}(\beta) e^{2 i n k z}
$$

will evolve as

$$
\Psi(t)=\sum_{n} J_{n} \beta e^{2 i n k z} e^{i E_{n} t / \hbar},
$$

where

$$
E_{n}=\frac{p^{2}}{2 m}=\frac{4 n^{2} k^{2} \hbar^{2}}{2 m}=4 n^{2} \hbar \omega_{\text {recoil }} .
$$

In this way, we can excite $\pm 2 \hbar k$ packets with up to $67 \%$ of the atoms from the source condensate involved. Even further improvements can be achieved by applying two such pulses, as demonstrated in [66] where nearly perfect splitting was observed.

Assuming that there is no axial potential, the wavepackets are allowed to propagate apart, until another pulse is applied to turn them around. This reflection pulse is longer in duration and achieves momentum reversal through normal Bragg scattering of the 


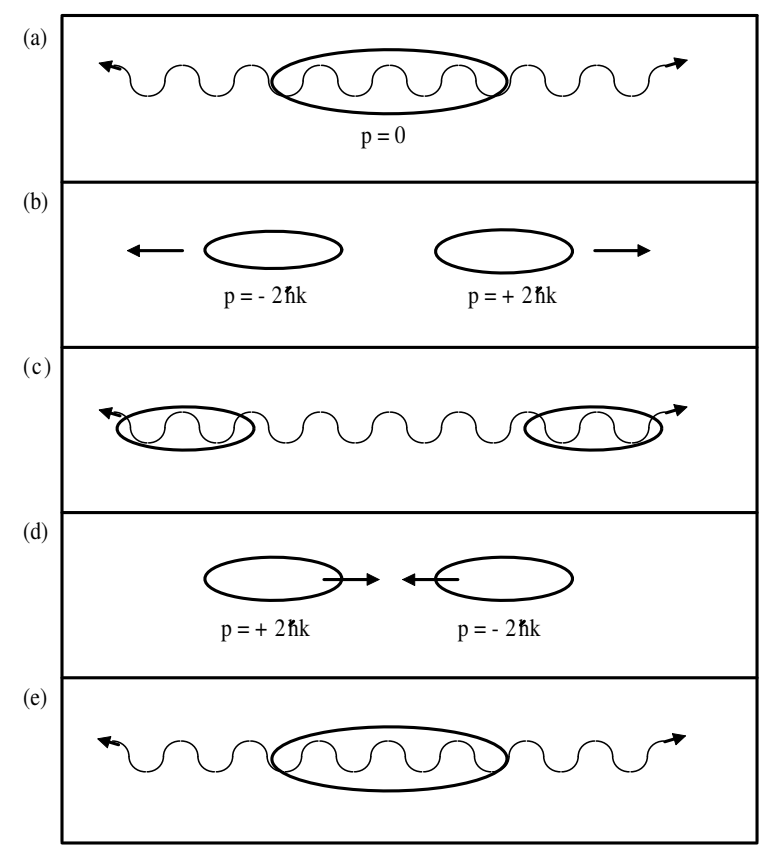

Figure 4.14: Splitting and recombining of condensate wavepackets. The initial doubles pulse splits the source condensate into two wavepackets with momentum $\pm \hbar k$. The two wavepackets propagate apart. Their momenta are reversed by the application of a Bragg pulse. When the two wavepackets have returned to their original position, another double laser pulse recombines them.

atoms off of the light beam [40]. Once the wavepackets return to the point of origin, they are recombined through the same sequence of laser pulses that was used to split them. Figure 4.14 displays a simple cartoon of the splitting and recombining process. We expect our ultimate interferometer to operate with this basic scheme. Indeed, as of this writing the splitting, reflection and recombination operations have been implemented, and an interference signal observed. Another possibility would be to reflect the condensates using the residual axial confinement observed in our waveguide. Alternatively, if the residual field causes spurious oscillations, we could cancel this field with the application of an appropriate external axial field. In any case we expect the detailed knowledge of the guide potential presented here to be useful. 


\section{Chapter 5}

\section{Conclusion}

The experiment described in this dissertation has three principle results. First, we have assembled and operated a BEC production machine based on the method of Lewandowski et al. [36], using two vacuum chambers isolated from each other by a thin tube. A MOT is operated in the first chamber, where atoms are captured out of a thermal vapor and cooled to about $200 \mu \mathrm{K}$. The atoms are then transferred to a magnetic trap which is mounted to a movable stage. A programmable motor moves the stage about half a meter, carrying the atoms to the second vacuum chamber where they are evaporatively cooled. We successfully used this apparatus to observe the first Bose-Einstein condensates at the University of Virginia.

Second, we have implemented a novel atom trap for Bose-Einstein condensates of ${ }^{87} \mathrm{Rb}$ to be used in atom interferometry experiments. The trap is based on a time-orbiting potential waveguide. It supports the atoms against gravity while providing weak confinement to minimize interaction effects. We have loaded a condensate into the waveguide, and removed all other confinement fields. Up to $2 \times 10^{4}$ condensate atoms have been loaded into the trap, at estimated temperatures as low as $850 \mathrm{pK}$. We expect this novel type of 
trap will be useful for a variety of applications in condensate interferometry.

Finally, we have characterized our trap by perturbing the atomic cloud with a sudden change in the confinement field. We subsequently obtain harmonic oscillation frequencies $\left(\omega_{x}, \omega_{y}, \omega_{z}\right)$ as low as $2 \pi \times(6.0,1.2,3.3) \mathrm{Hz}$. We have modified our mathematical description of the waveguide fields to account for the residual fields from the trap leads, obtaining good agreement between the measured and predicted trap behavior. We anticipate that interferometer measurement times of $1 \mathrm{~s}$ or more should be achievable in this device.

The weak confinement of our guide should greatly reduce the limiting effects of interactions. For instance, the phase distortions discussed in [48] should have negligible effect for condensate numbers below about $1.5 \times 10^{4}$, and phase diffusion effects during the wavepacket propagation should not become important for interaction times less than about $1 \mathrm{~s}$ [32]. Using the current apparatus, we plan to study these and other limiting effects. With suitable modifications, our waveguide could be used to precisely measure electric polarizability, gravitational forces, rotations, and other phenomena [10]. We expect that the trap design presented here will play an essential role in allowing condensate interferometry to realize its potential. 
Appendices 


\section{Appendix A}

\section{Tables of Constants}

\begin{tabular}{||l|c|c|c||}
\hline Constant & Symbol & Value & Units \\
\hline Speed of Light & $c$ & $2.99792458 \times 10^{8}$ & $\mathrm{~m} / \mathrm{s}$ \\
Planck's Constant & $h$ & $6.62606876 \times 10^{-34}$ & $\mathrm{Js}$ \\
Planck's Constant & $\hbar$ & $1.054571596 \times 10^{-34}$ & $\mathrm{Js}$ \\
Boltzmann's Constant & $k_{B}$ & $1.3806503 \times 10^{-23}$ & $\mathrm{~J} / \mathrm{K}$ \\
Electron Charge & $e$ & $1.602176462 \times 10^{-19}$ & $\mathrm{C}$ \\
Electron Mass & $m_{e}$ & $9.10938188 \times 10^{-31}$ & $\mathrm{~kg}$ \\
Atomic Mass Unit & $\mathrm{u}$ & $1.66053873 \times 10^{-27}$ & $\mathrm{~kg}$ \\
Bohr Magneton & $\mu_{B}$ & $9.27400899 \times 10^{-24}$ & $\mathrm{~J} / \mathrm{T}$ \\
Bohr Radius & $a_{0}$ & $0.5291772083 \times 10^{-10}$ & $\mathrm{~m}$ \\
Permittivity of Vacuum & $\epsilon_{0}$ & $8.854187817 \times 10^{-12}$ & $\mathrm{C}^{2} / \mathrm{N}-\mathrm{m}^{2}$ \\
Permeability of Vacuum & $\mu_{0}$ & $4 \pi \times 10^{-7}$ & $\mathrm{~N} / \mathrm{A}^{2}$ \\
\hline
\end{tabular}

Table A.1: Exact values of frequently used constants.

\begin{tabular}{||l|c|c|c||}
\hline Constant & Symbol & Value & Units \\
\hline Atomic Mass & $m$ & 86.909180520 & $\mathrm{u}$ \\
Scattering Length & $a$ & 5.77 & $\mathrm{~nm}$ \\
Nuclear Spin & $I$ & $3 / 2$ & \\
\hline
\end{tabular}

Table A.2: Physical Properties of ${ }^{87} \mathrm{Rb}$. 

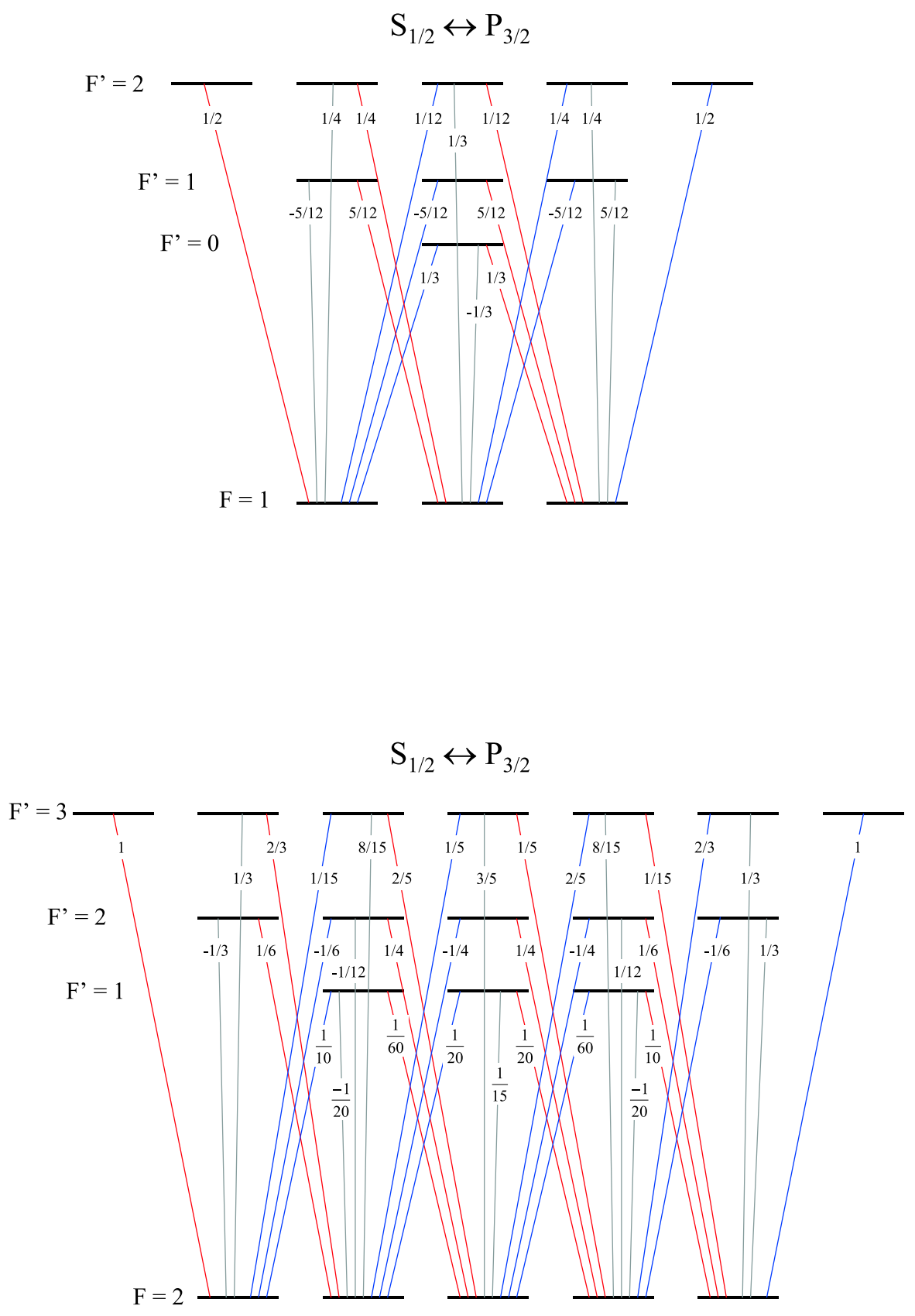

Figure A.1: Coupling constants for ${ }^{87} \mathrm{Rb}$. 

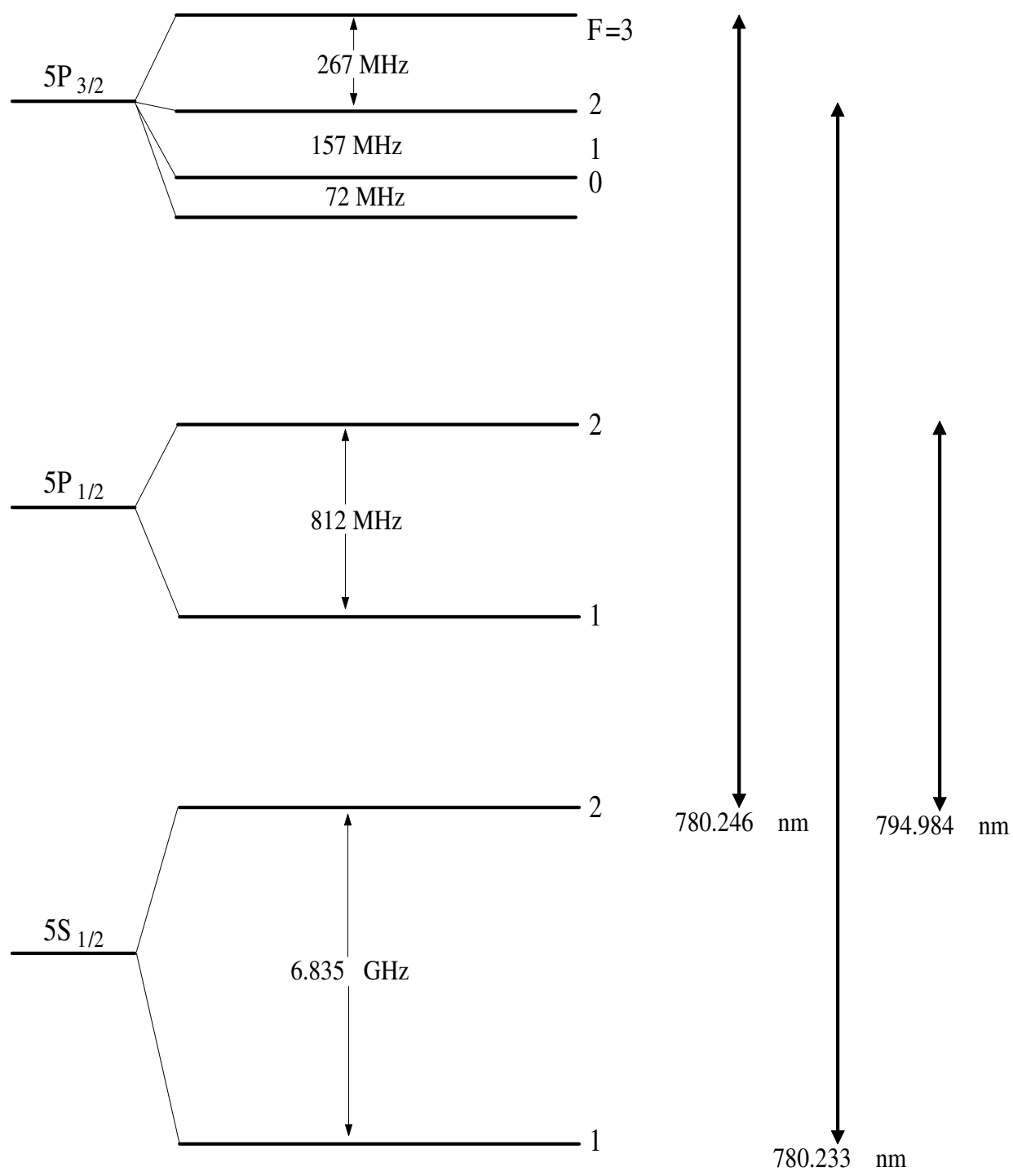

Figure A.2: Energy level diagram for ${ }^{87} \mathrm{Rb}$. 


\begin{tabular}{||l|c|c|c||}
\hline Constant & Symbol & Value & Units \\
\hline Wavelength & $\lambda$ & 780.241209686 & $\mathrm{~nm}$ \\
Lifetime & $\tau$ & 26.24 & $\mathrm{~ns}$ \\
Natural Linewidth & $\Gamma$ & $2 \pi \times 6.065$ & $\mathrm{MHz}$ \\
Recoil velocity $=\frac{\hbar k}{m}$ & $\mathrm{v}_{r}$ & 5.8845 & $\mathrm{~mm} / \mathrm{s}$ \\
Recoil temperature $=\frac{\hbar^{2} k^{2}}{m k_{B}}$ & $T_{r}$ & 361.96 & $\mathrm{nK}$ \\
Doppler temperature $=\frac{\hbar \Gamma}{2 k_{B}}$ & $T_{D}$ & 146 & $\mu \mathrm{K}$ \\
Electron spin $g$-factor & $g_{S}$ & 2.0023193043737 & \\
Electron orbital $g$-factor & $g_{L}$ & 0.99999369 & \\
Fine structure Landé & $g_{J}\left(5^{2} S_{1 / 2}\right)$ & 2.00233113 & \\
$g$-factor & $g_{J}\left(5^{2} P_{3 / 2}\right)$ & 1.3362 & \\
Nuclear Landé $g$-factor & $g_{I}$ & -0.0009951414 & \\
\hline
\end{tabular}

Table A.3: ${ }^{87} \mathrm{Rb} \mathrm{D}_{2}\left(5^{2} \mathrm{~S}_{1 / 2} \leftrightarrow 5^{2} \mathrm{P}_{3 / 2}\right)$ Transition Properties. Rubidium line data obtained from [5].

\begin{tabular}{||l|c||}
\hline Polarization & Saturation Intensity \\
\hline isotropic & $3.576 \mathrm{~mW} / \mathrm{cm}^{2}$ \\
$\pi$ & $2.503 \mathrm{~mW} / \mathrm{cm}^{2}$ \\
$\sigma^{ \pm}$ & $1.669 \mathrm{~mW} / \mathrm{cm}^{2}$ \\
\hline
\end{tabular}

Table A.4: Saturation intensity for $F=2 \leftrightarrow F^{\prime}=3$ transition of ${ }^{87} \mathrm{Rb}$. Rubidium line data obtained from [5], where the scattering rate is defined as $R_{s}=\frac{\Gamma}{2} \frac{I / I_{s}}{1+2\left(\frac{\Omega}{\Gamma}\right)^{2}+4\left(\frac{\Delta}{\Gamma}\right)^{2}}$ for $I / I_{s}=2\left(\frac{\Omega}{\Gamma}\right)^{2}$. 


\section{Appendix B}

\section{EOM Driver}

Our electro-optic modulator (EOM) requires an RF driver. While such a device can be purchased, the cost can be significant, and so we have constructed our own. The heart of the driver is illustrated by the circuit diagram in Figure B.1. A VCO at $3.3 \mathrm{GHz}$ generates the main RF signal, which then passes to a doubler. This is where the $6.6 \mathrm{GHz}$ signal described in Chapter 2 originates. The RF signal is transmitted then to the microwave cavity within the EOM itself. A Pasternack PE2204-20 directional coupler sends a small portion of the reflected RF signal into the lock circuit shown in Figure B.2 for frequency interrogation and locking. The main RF signal is mixed with a $10 \mathrm{MHz}$ modulation signal to assist in frequency locking. The lock circuit is contained within the circuit box shown in Figure B.1. The frequency lock is necessary because our EOM operates at a relatively high frequency which requires use of a microwave resonator. Since the resonance frequency can drift, our lock keeps the operating frequency at the resonance of the cavity.

We control the efficiency of the EOM through a bias input to the $3.3 \mathrm{GHz} \mathrm{VCO}$. By attenuating the output of the VCO, we reduce the RF amplitude. Figure B.3 shows the bias control circuit, and the $10 \mathrm{MHz}$ VCO that produces the modulation for the lock 


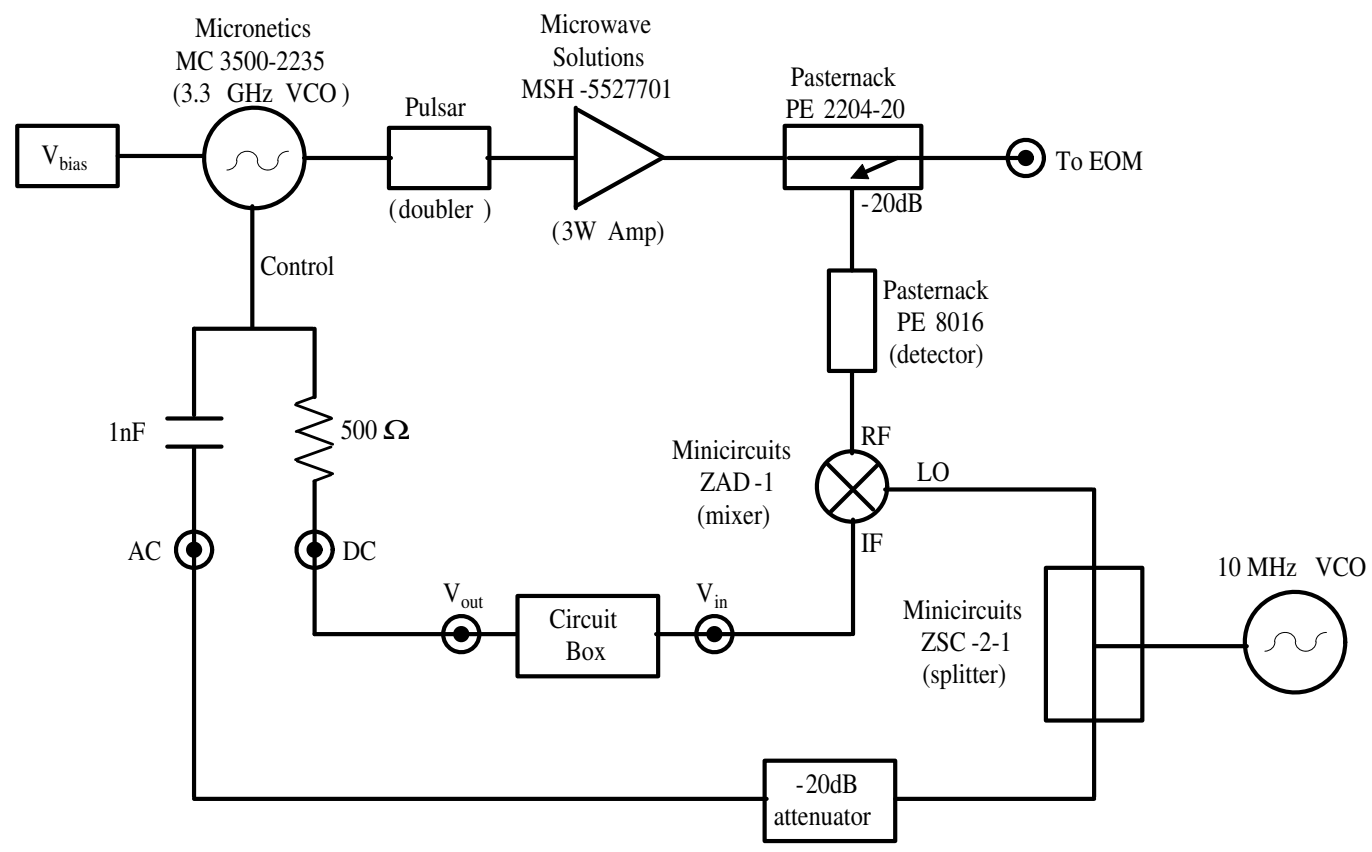

Figure B.1: Circuit diagram for generation of RF signal to drive EOM.

signal. When we were working on producing condensates but not yet using the atom waveguide, we controlled the VCO bias with an analog channel on our ADwin pulsing system. When we later need that analog channel to control the linear quadrupole field of the waveguide, we moved the VCO bias control to a digital channel. The switch shown at the input to the bias circuit allows us to accommodate use of either a digital or an analog channel to control the VCO bias. By passing the input signal directly to the transistor, we can simply use an analog signal from the ADwin system to select the desired amplitude of EOM operation. Here, a $0 \mathrm{~V}$ signal turned the EOM on full, a $5 \mathrm{~V}$ signal turned it full off, and a $2.2 \mathrm{~V}$ signal was used during the CMOT stage to turn the EOM approximately halfway on. When we later switched channels so that the EOM was now controlled by a digital signal, we added the potentiometer option to the bias control. 


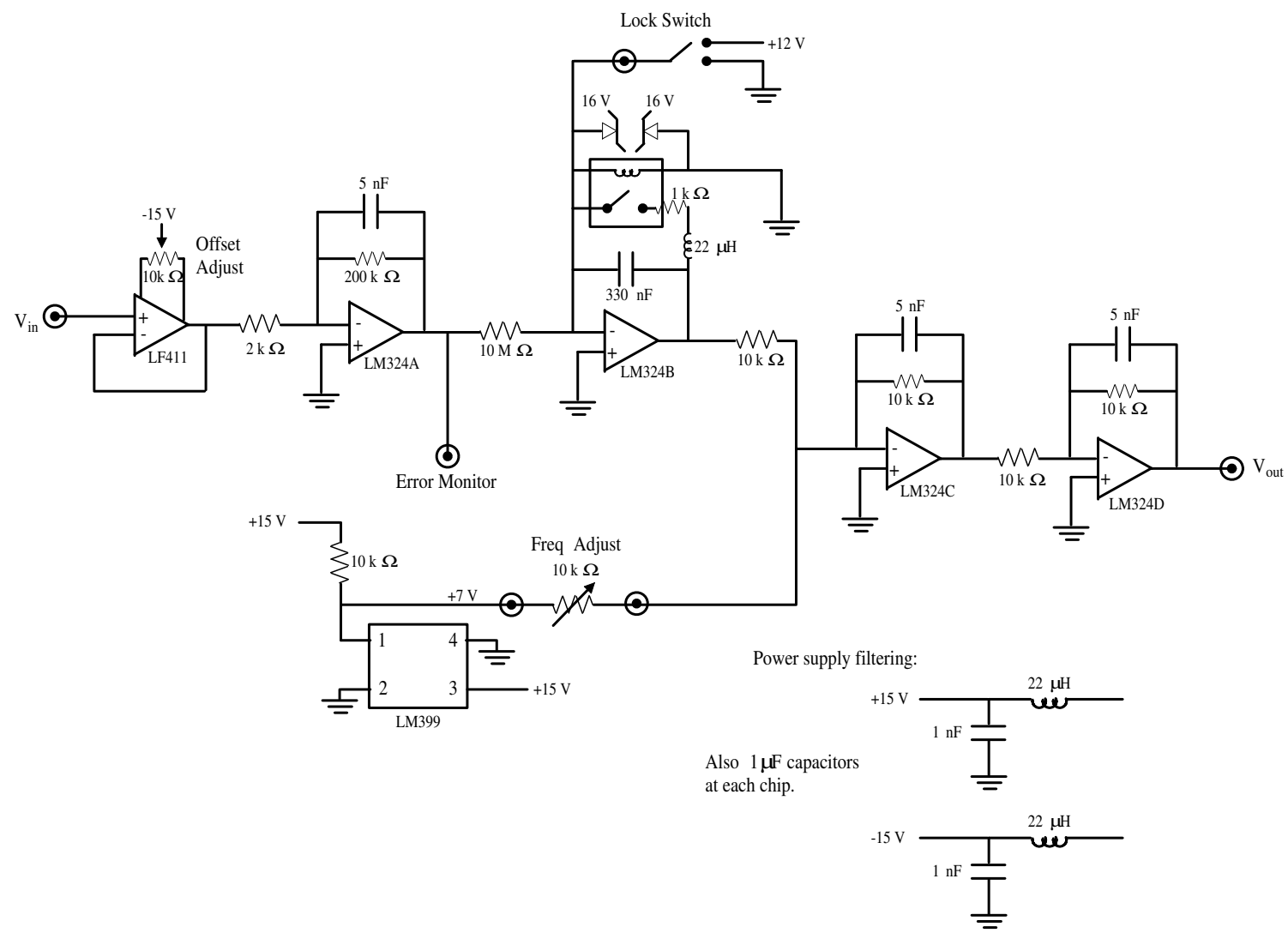

Figure B.2: Circuit diagram for EOM frequency lock. 

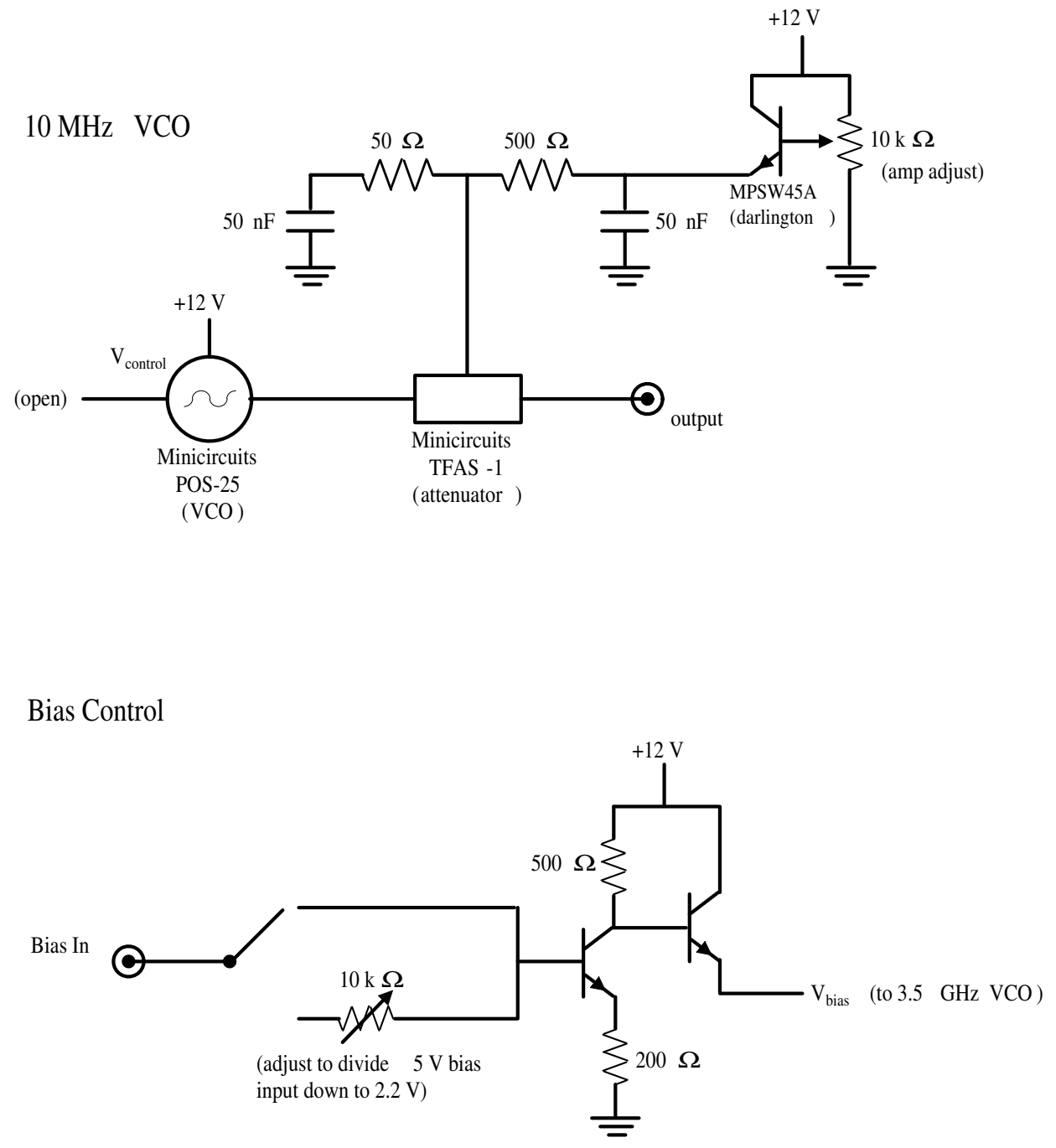

Figure B.3: Circuit diagrams for $10 \mathrm{MHz}$ modulation and bias control. The switch at the input to the bias control circuit allows used of either analog or digital control voltages, by transmitting the signal either directly to the transistor (for analog input) or first passing through a potentiometer (for digital input). 


\section{Appendix C}

\section{AOM Driver}

We also constructed RF drivers to control the acousto-optic modulators (AOM's) throughout our system. We have both fixed and variable frequency RF drivers. The simplest control panel is the fixed-frequency RF driver, shown in Figure C.1. While the Mod Input allows for either an analog or digital control voltage from the computer, the ALC input can be used in conjunction with it to increase the switching speed for analog signals. Both inputs are connected to a Crystal Technology model 1080-25 AOM driver that sends an $80 \mathrm{MHz}$ signal to the Crystal Technology model 3080-122 AOM.

We also designed our own variable-frequency RF driver, as shown in Figure C.2. A Minicircuits ZOS-100 VCO generates the RF signal, which is sent through a Minicircuits ZHL-3A RF amplifier and then passed to an AOM. The amplitude of the RF signal is modified by applying a control voltage to a Minicircuits ZAS-3 RF attenuator. The frequency of the RF signal can be modified by either applying a control voltage or adjusting the dial of a $5 \mathrm{k} \Omega$ potentiometer. The frequency of the $\mathrm{VCO}$ can be sampled using its auxiliary output and a frequency counter. 


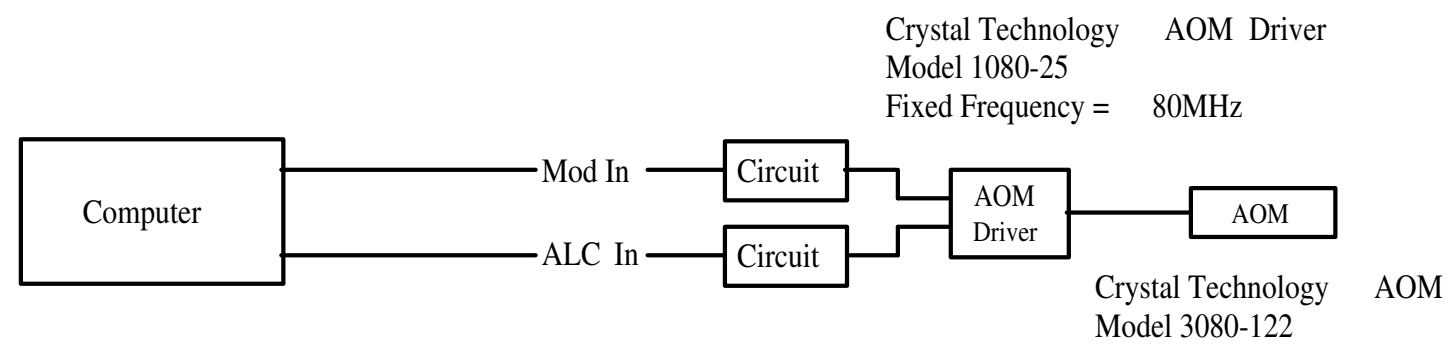

Mod In Circuit:

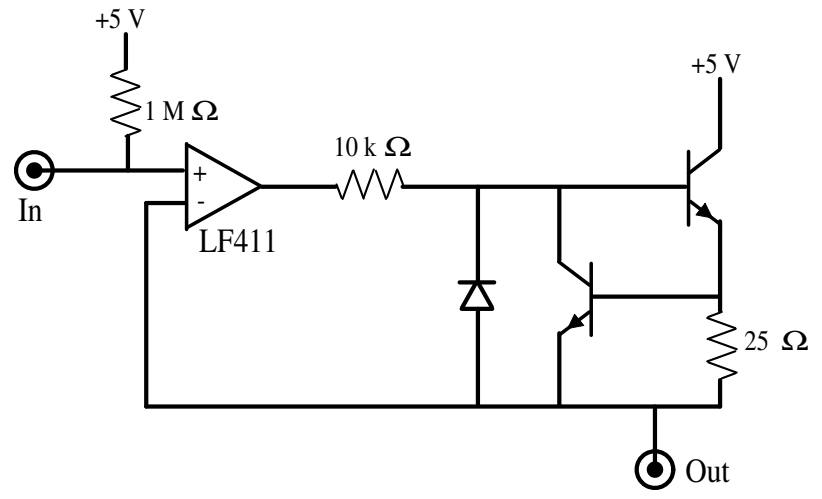

ALC In Circuit:

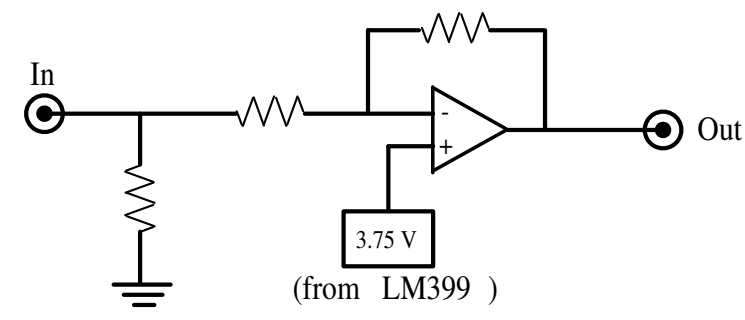

Figure C.1: Circuit diagrams for fixed-frequency AOM driver. 


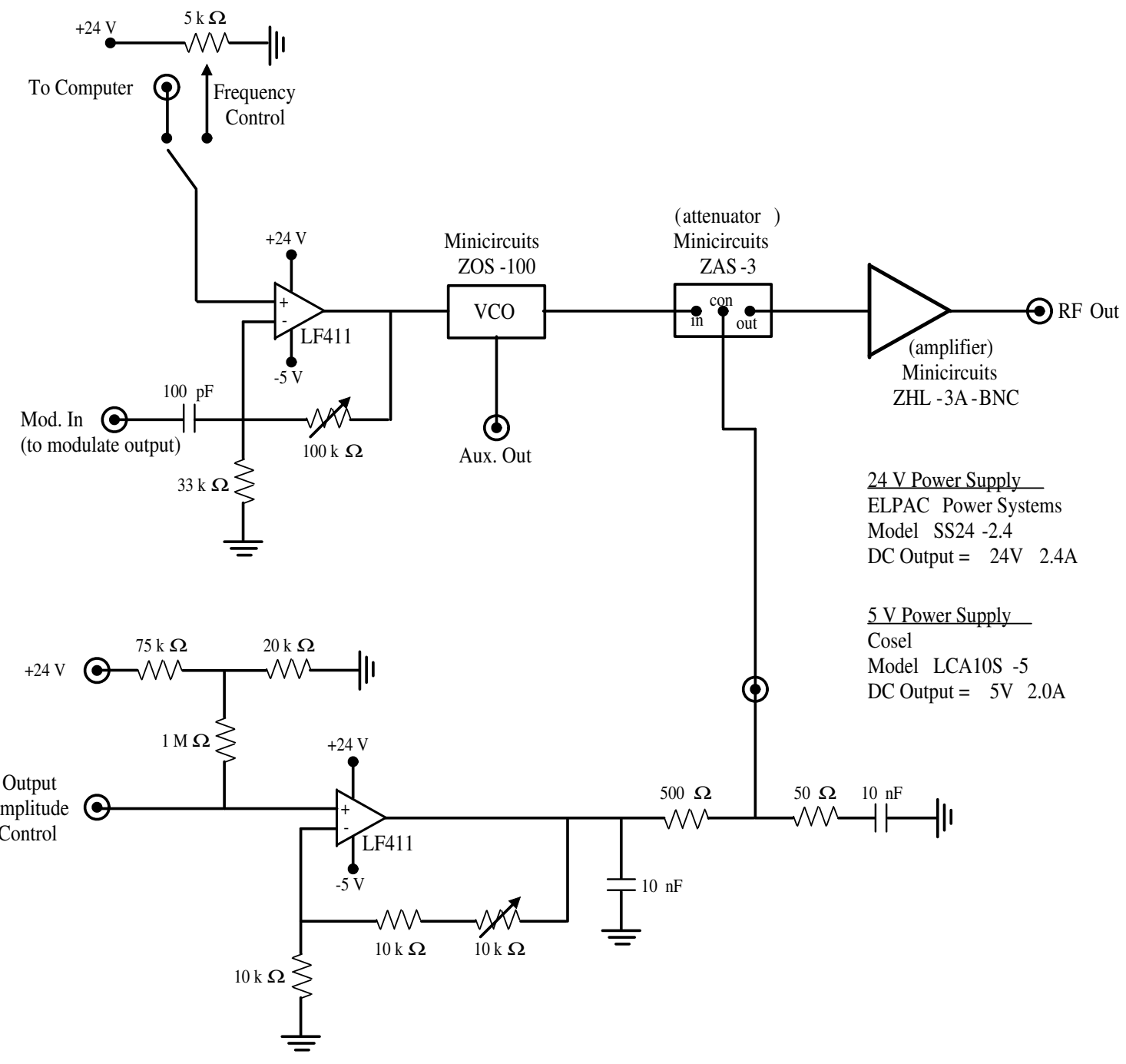

Figure C.2: Circuit diagram for variable-frequency AOM driver. 


\section{Appendix D}

\section{Lock-In Amplifier}

We constructed our own lock-in amplifier to run the saturated absorption lock used to stabilize the frequency of the Ti-Sapphire laser. It is based on an Analog Devices AD630JN demodulator chip, which is appropriate for applications such as balanced modulation and demodulation, phase-sensitive detection, lock-in amplification, and square wave multiplication. According to the datasheet, this device can recover a signal from $100 \mathrm{~dB}$ of noise.

We constructed our lock-in amplifier from the circuit design suggested by the AD630JN datasheet. The main circuit, including the AD630JN, is shown in Figure D.2 as the "demodulator." This device accepts the signal from a photodiode, and outputs a dispersiveshaped error signal. Using an integrator, the laser frequency can be held at the zerocrossing of the error signal.

Figure D.3 shows the circuit diagram for the modulation source. This signal oscillates the current through the solenoid surrounding the saturated absorption vapor cell, resulting in modulation of the Zeeman splittings of the Rb atoms. This same modulation signal is sent through a phase shifter and an AC coupler, and then passed to the Reference In pin 
of the AD630JN. The AD630JN is optimized for operation at $1 \mathrm{kHz}$, and we generated the modulation signal with a function generator set to that frequency. The phase shifter includes a potentiometer, so that the phase of the reference signal can be adjusted as needed to produce a dispersive error signal.

While the photodiode and integrator are not unique to this circuit, I have included their designs for completeness. The circuit diagram for each is shown in Figure D.1.
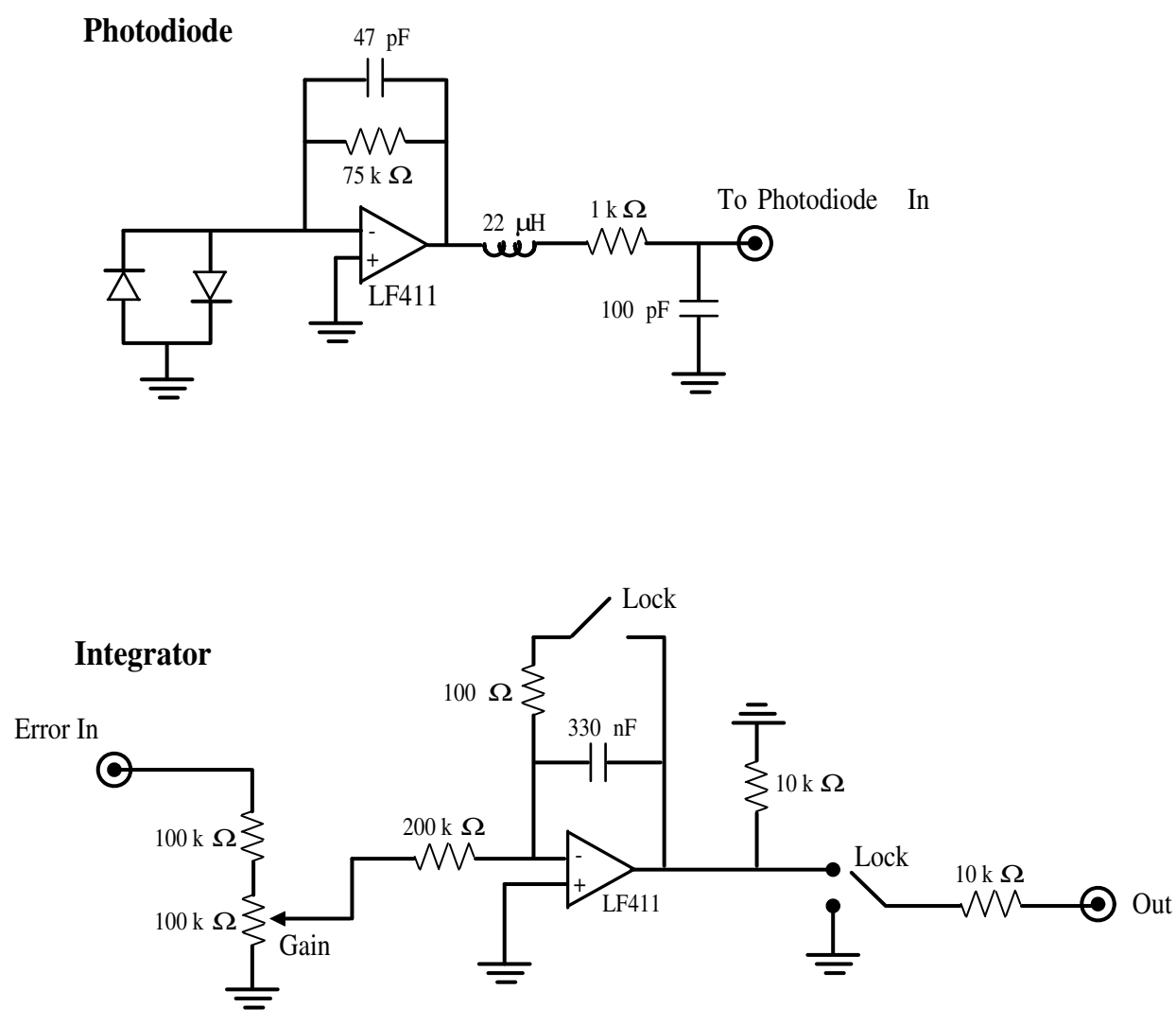

Figure D.1: Circuit diagrams for photodiode to collect saturated absorption signal, and integrator to produce lock signal. 


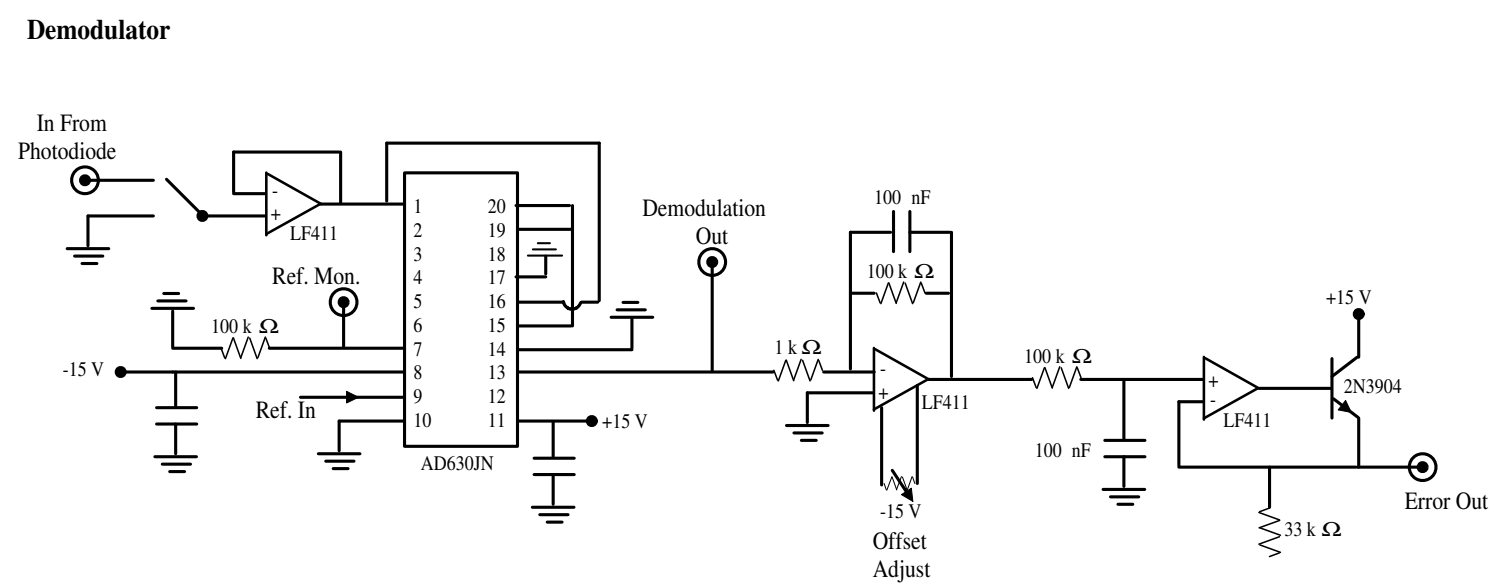

Figure D.2: Circuit diagram for demodulator.
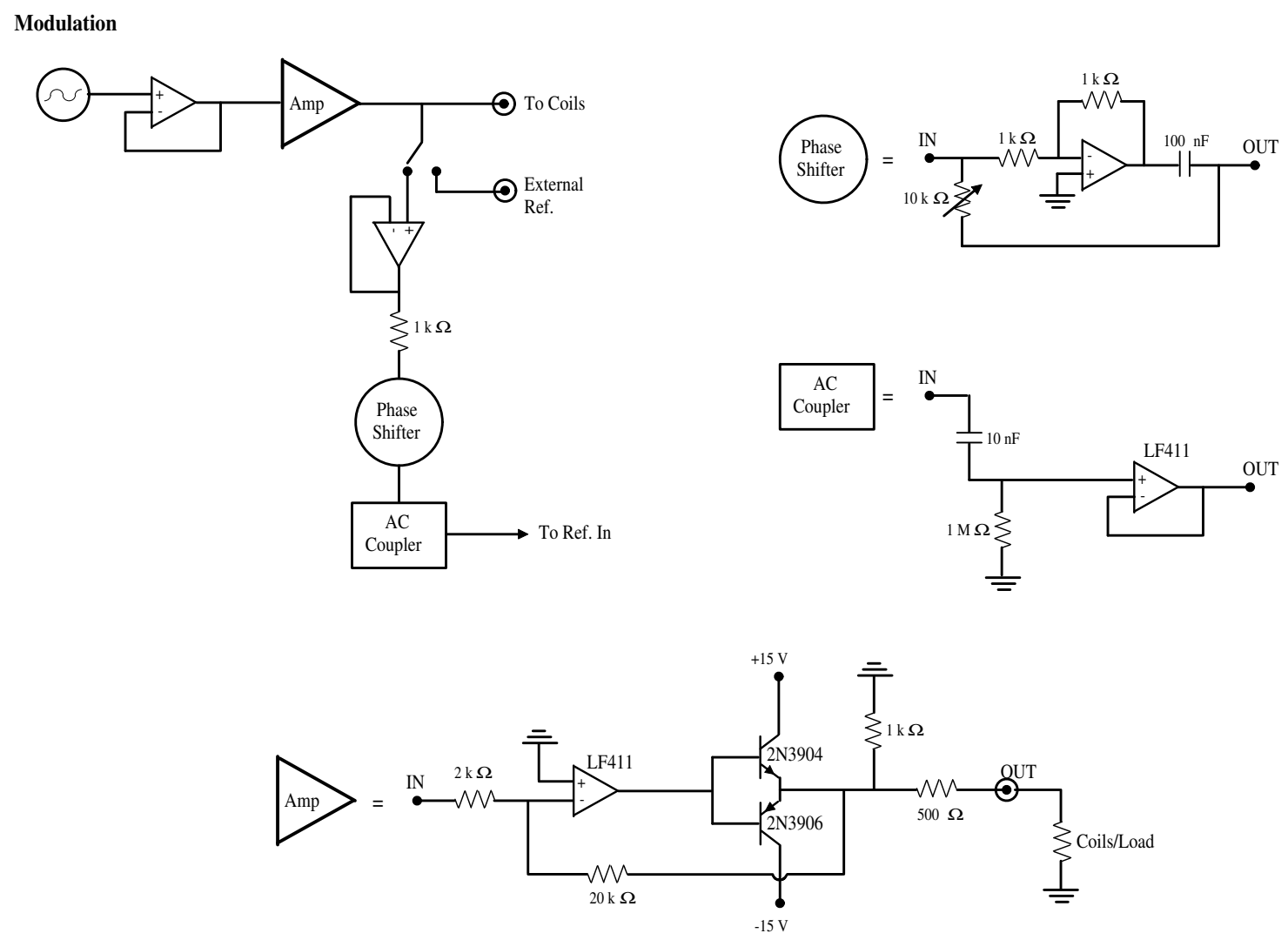

Figure D.3: Circuit diagram for modulation of magnetic field, and modulation reference for lock-in amplifier. 


\section{Appendix E}

\section{Coil Holder Assembly}

Our coilholder assembly consists of three pieces. The first piece, shown in Figure E.1, is the coilholders themselves. We had two coilholders machined from Delrin according to the instructions in the schematic. We had to drill a small hole in the bottom of each coilholder to allow the installation of a small piece of tubing that would bring the chilled water into the coils. The end of this patch tubing was cut at a $45^{\circ}$ angle and soldered to the end of the coiled tubing, also cut at an angle. The coils were then epoxied in place within the coilholders.

The coilholders were then mounted onto an aluminum tower. The ends of the coilholders contain three 8-32 tapped holes. The tower has three corresponding vertical slots, as shown in the schematic in Figure E.2. The coilholders can be slid vertically along these slots, in order to select proper placement of the coils with regard to the vacuum chamber. Once in place, the screws are tightened to secure coilholder position. We found that it was important to use washers between the screws and the slots in order to avoid slowly deforming the slots if the screws happened to be over-tightened.

Finally, the aluminum tower must be secured onto the translation carriage. The 
factory-made mounting holes on the carriage were used to attach an aluminum plate. The plate was machined with four 8-32 holes matching the bottom of the aluminum tower. The design of the aluminum plate is shown in Figure E.3. The final assembly with aluminum plate, tower, and coilholders is shown in Chapter 3.
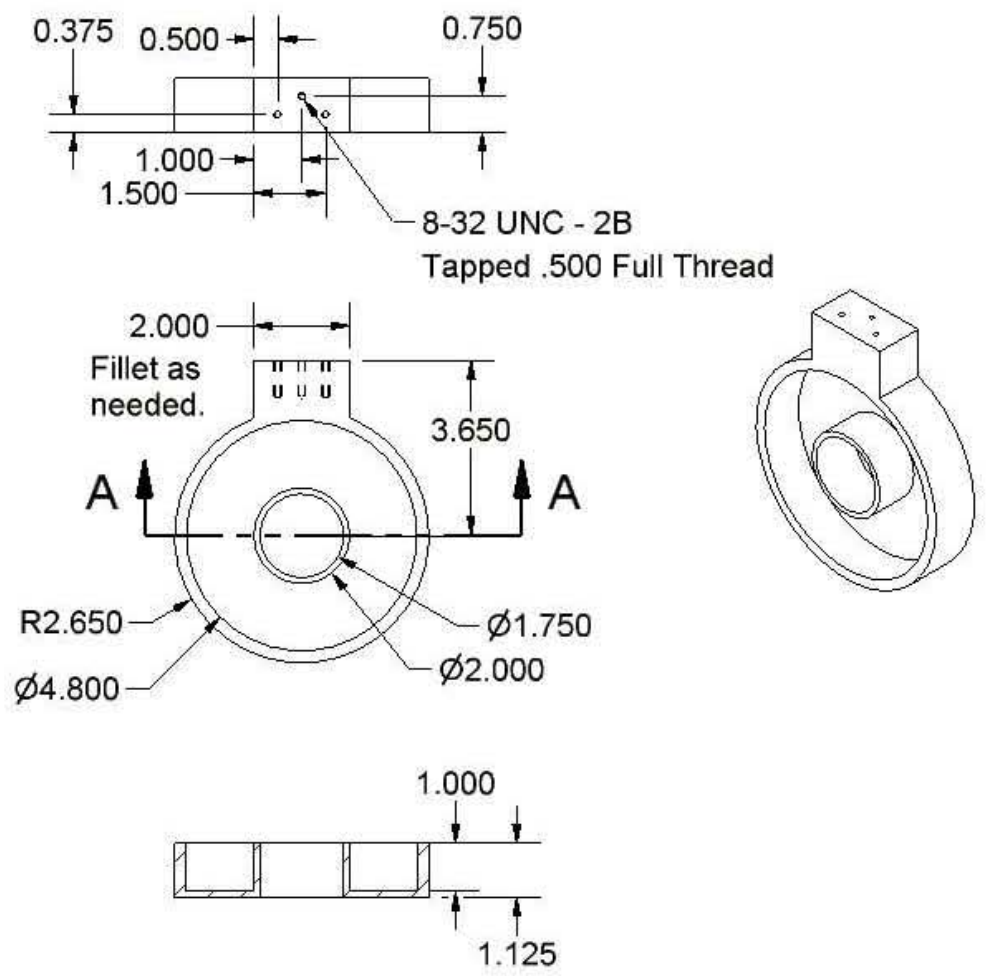

Figure E.1: Drawing of Delrin coilholder. 

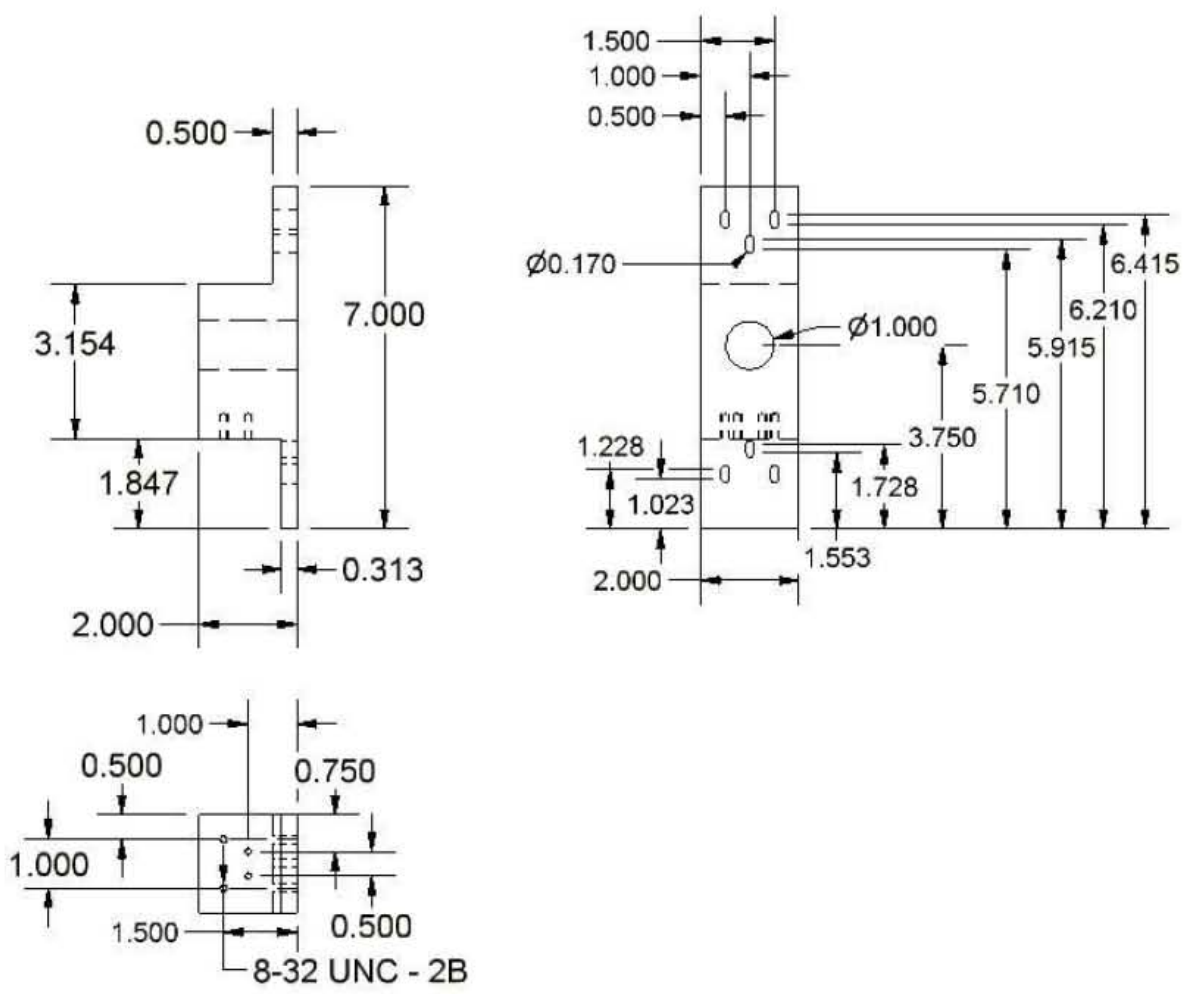

Figure E.2: Drawing of aluminum support tower.

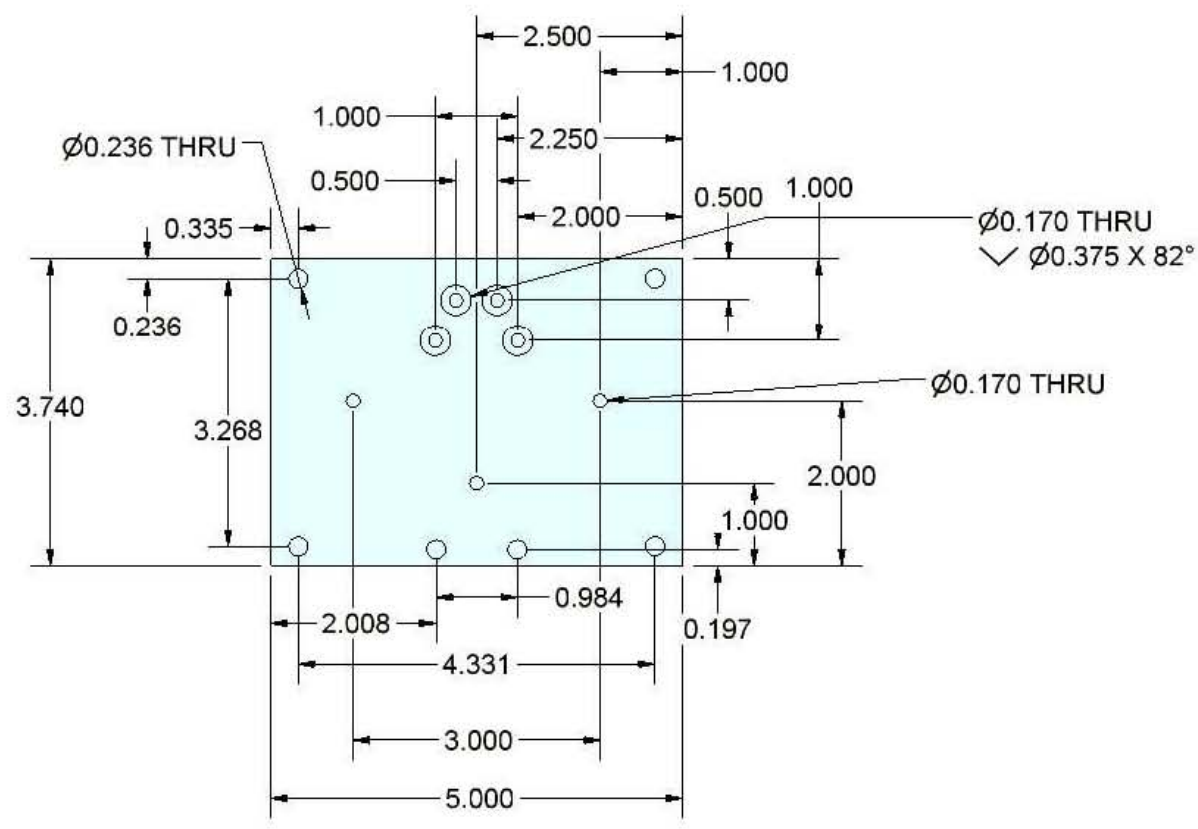

Figure E.3: Drawing of aluminum mounting plate. 


\section{Appendix F}

\section{Pulse Sequences}

\begin{tabular}{||c|c||}
\hline Channel & Function \\
\hline$a$ & trapping and repump beams on/off \\
$c$ & camera trigger \\
$d$ & main B field on/off \\
$e$ & TOP fields on/off \\
$f$ & main shutter \\
$g$ & bias field on/off \\
$h$ & move trap (reverse logic) \\
$i$ & double-pass AOM on/off \\
$j$ & GPIB command \\
$k$ & diode repumper beam on/off \\
$l$ & probe beam on/off \\
Analog1 & optical pumping beam on/off \\
Analog2 & double-pass AOM frequency \\
Analog3 & main B field current control \\
Analog4 & EOM VCO bias control \\
\hline
\end{tabular}

Table F.1: Channel Assignment.

The assignment of channels for our ADwin pulsing system is shown in Table F.1. Channels a through $l$ are digital, so that $0 \mathrm{~V}$ is applied when the channel is off and $5 \mathrm{~V}$ is 


\begin{tabular}{|c|c|c|c|c|c|c|c|}
\hline Pulse & Length (ms) & Dig. Out & A1 & A2 & A3 & A4 & Action \\
\hline 1 & loadcmot & & & & & & MOT, CMOT, optical molasses, optical pumping \\
\hline 2 & movetrap & & & & & & load B trap, move trap to science cell \\
\hline 3 & 500 & $\mathrm{ch}$ & 0 & 10 & 0 & 0 & wait (ensure trap has come to rest) \\
\hline 4 & $\mathrm{rs} 232 \mathrm{i} \mathrm{b}$ & $\mathrm{ch}$ & & & & & evaporation stages 1 and 2 \\
\hline 5 & 500 & $\mathrm{ch}$ & 0 & 10 & 0 & 0 & wait \\
\hline 6 & 500 & chd & 0 & $"$ & 0 & 5 & turn on bias field to create TOP trap \\
\hline 7 & $5000 \mathrm{r}$ & chd & 0 & $"$ & 0 & 0.5 & ramp down TOP fields \\
\hline 8 & rs232 i g & chd & & & & & evaporation stage 3 \\
\hline 9 & $1000 \mathrm{r}$ & chd & 0 & 10 & 0 & -1 & ramp down TOP fields \\
\hline 10 & $8000 \mathrm{r}$ & chd & 0 & 10 & 0 & -3 & ramp down TOP fields (slowly) \\
\hline 11 & $\mathrm{rs} 232 \mathrm{i} \mathrm{j}$ & chd & & & & & evaporation stages 4 and 5 \\
\hline 12 & probe & & & & & & image condensate \\
\hline 13 & 16000 & $\mathrm{~h}$ & 0 & 0 & 0 & 0 & download image from CCD \\
\hline 14 & $500 \mathrm{r}$ & $\mathrm{ch}$ & 0 & 10 & 0 & 0 & ramp main B field to full strength \\
\hline 15 & probe & & & & & & "no atoms" image \\
\hline 16 & 1850 & g & 0 & 0 & 0 & 0 & move trap back to MOT chamber \\
\hline 17 & 100 & aceghj & 0.05 & 0.25 & 0 & -5 & MOT \\
\hline
\end{tabular}

Table F.2: Pulse sequence used to observe condensate.

applied when the channel is on. $A 1-A 4$ are analog channels where the control voltage can be set from $0-10 \mathrm{~V}$.

The pulse sequence used to observe our first Bose-Einstein condensates is shown in Table F.2. The length of each pulse, the channels activated, and the action taken are included. The ADwin system allows inclusion of subroutine pulse sequences, which are detailed in Tables F.3, F.4 and F.5. Other shorthand notations include adding an $r$ to the pulse length to command the analog channels to ramp from the previous voltage to the voltage set in the current step, and use of quotation marks to leave a command unchanged from the previous step.

In making our early condensates, we used five stages of evaporative cooling. The parameters of each stage are shown in Table F.6. Table F.7 shows the pulse sequence used to make a condensate and then load it into the waveguide. By the time we started using the waveguide, we had been making condensates for over a month and had made 


\begin{tabular}{||c|c|c|c|c|c|c|c||}
\hline \multicolumn{9}{||c|}{ Subroutine: loadcmot } \\
\hline Pulse & Length (ms) & Dig. Out & A1 & A2 & A3 & A4 & Action \\
\hline 1 & 98 & aceghj & 0.05 & 0.25 & 0 & 0 & MOT \\
2 & 15 & acegh & 0.5 & 0.25 & 2.2 & 0 & CMOT \\
3 & 1 & aegh & 0.6 & 0 & 2.2 & 0 & optical molasses \\
4 & 0.2 & efghj & 0 & 10 & 5 & 0 & prepare for B field catch \\
5 & 1 & efghjl & 0.5 & 10 & 5 & 0 & optical pumping, prepare B field \\
\hline
\end{tabular}

Table F.3: Pulse sequence for subroutine loadcmot.

\begin{tabular}{||c|c|c|c|c|c|c|c||}
\hline \multicolumn{9}{||c|}{ A } \\
\hline Pulse & Length (ms) & Dig. Out & A1 & A2 & A3 & A4 & Action \\
\hline 1 & 4 & cg & -1.2 & 3.2 & 5 & -5 & initiate magnetic catch \\
2 & 1 & cgj & -1.2 & 3.2 & 5 & -5 & extra repump and catch \\
3 & 50 & cg & -1.2 & 3.2 & 5 & -5 & hold in weak B trap \\
4 & $200 \mathrm{r}$ & $\mathrm{cg}$ & -1.2 & 10 & 5 & -5 & compress trap \\
5 & 1850 & $\mathrm{c}$ & 0 & 10 & 0 & -5 & move trap to science cell \\
\hline
\end{tabular}

Table F.4: Pulse sequence for subroutine movetrap.

\begin{tabular}{||c|c|c|c|c|c|c|c|c||}
\hline \multicolumn{9}{||c|}{} \\
\hline Pulse & Length (ms) & Dig. Out & A1 & A2 & A3 & A4 & Action \\
\hline 1 & 30 & bcde & 0 & 10 & 5 & -3 & open main shutter \\
2 & 20 & beh & -0.55 & 0 & 5 & 0 & ballistic expansion \\
3 & 0.05 & behkl & $"$ & 0 & 5 & 0 & probe \\
4 & 0.1 & ebh & $"$ & 0 & 5 & 0 & extra time \\
\hline
\end{tabular}

Table F.5: Pulse sequence for subroutine probe.

some modifications to that part of the pulse sequence. Also, we needed to use one of the analog channels to control the amplitude of the waveguide quadrupole field. We moved the EOM bias control to digital channel $l$, thereby freeing $A 3$ for assignment to the waveguide field. This was also when we modified the EOM driver panel to accept a digital input instead of analog, by adding a switch and voltage divider to the bias input on the VCO that produced the $3.3 \mathrm{GHz} \mathrm{RF}$.

The optical pumping beam, formerly controlled by channel $l$, we now controlled with a pulse delay panel and an AND gate. The pulse delay system was constructed using a dual retriggerable one-shot from Fairchild Semiconductor, model DM74LS123N. The one-shot was wired according to the suggestions in its datasheet, in order to produce a 


\begin{tabular}{||c|c|c|c|c||}
\hline \multicolumn{5}{||c||}{ routine b } \\
\hline$\nu_{\text {start }}(\mathrm{MHz})$ & $\nu_{\text {stop }}(\mathrm{MHz})$ & $\tau(\mathrm{s})$ & $\mathrm{RF}$ Power $(\mathrm{dBm})$ & $\nu_{\text {bottom }}(\mathrm{MHz})$ \\
\hline 50 & 30 & 12 & -13 & 0 \\
30 & 15 & 6 & -13 & 0 \\
\hline \multicolumn{5}{|c||}{ routine g } \\
\hline 10.4 & 4.4 & 6 & -13 & 7.6 \\
\hline \multicolumn{5}{|c|}{ routine j } \\
\hline 1.85 & 0.65 & 6 & -18 & 2.55 \\
0.65 & 0.2 & 3 & -18 & 2.55 \\
\hline
\end{tabular}

Table F.6: Evaporation routines.

variable length pulse that followed a trigger pulse by a variable length delay. We used the optical pumping bias field as a trigger for the pulse delay panel. The panel receives the same signal used to turn on this bias field, and after $200 \mu$ s the panel outputs a $1 \mathrm{~ms}$ pulse. The bias field trigger and the $1 \mathrm{~ms}$ pulse from this panel are then passed through an AND gate, the output of which is passed to the optical pumper beam AOM. When both signal are present, the output of the AND gate is high, and the optical pumper beam is switched on. The AND gate trims the timing of the bias field and the optical pumper beam for maximum efficiency.

We also modified the "loadcmot" subroutine at this point, using digital control of the VCO bias for the operation of the EOM and the new method of activating the optical pumping beam. 


\begin{tabular}{|c|c|c|c|c|c|c|c|}
\hline Pulse & Length (ms) & Dig. Out & A1 & A2 & A3 & A4 & Action \\
\hline 1 & loadcmot2 & & & & & & MOT, CMOT, optical molasses, optical pumping \\
\hline 2 & movetrap & & & & & & load B trap, move trap to science cell \\
\hline 3 & 500 & $\mathrm{ch}$ & 0 & 10 & -5 & 0 & wait (ensure trap has come to rest) \\
\hline 4 & $\mathrm{rs} 232 \mathrm{i} \mathrm{b}$ & $\mathrm{ch}$ & & & & & evaporation stages 1 and 2 \\
\hline 5 & 500 & $\mathrm{ch}$ & 0 & 10 & -5 & 0 & wait \\
\hline 6 & 500 & chd & 0 & $"$ & $"$ & 0 & turn on bias field to create TOP trap \\
\hline 7 & $10000 \mathrm{r}$ & chd & 0 & 8 & $"$ & -3 & ramp down TOP and main $B$ fields \\
\hline 8 & $\mathrm{rs} 232 \mathrm{i} \mathrm{j}$ & chd & & & & & evaporation stages 4 and 5 \\
\hline 9 & $1000 \mathrm{r}$ & chd & 0 & 2 & 3 & -3 & ramp down main $B$ field \\
\hline 10 & $1000 \mathrm{r}$ & chd & 0 & 1 & $"$ & $"$ & ramp down B field (slowly) \\
\hline 11 & $5000 \mathrm{r}$ & chd & 0 & 0 & $"$ & $"$ & finish ramp, adiabatically load waveguide \\
\hline 12 & probe & & & & & & image condensate \\
\hline 13 & 16000 & $\mathrm{~h}$ & 0 & 0 & -5 & 0 & download image from CCD \\
\hline 14 & $500 \mathrm{r}$ & $\mathrm{ch}$ & 0 & 10 & -5 & 0 & ramp main B field to full strength \\
\hline 15 & probe & & & & & & "no atoms" image \\
\hline 16 & 1850 & $\mathrm{~g}$ & 0 & 0 & -5 & -5 & move trap back to MOT chamber \\
\hline 17 & 100 & aceghj & 0.05 & 0.25 & -5 & -5 & MOT \\
\hline
\end{tabular}

Table F.7: Pulse sequence used to load atoms into waveguide.

\begin{tabular}{||c|c|c|c|c|c|c|c||}
\hline \multicolumn{9}{||c|}{ Subroutine: loadcmot2 } \\
\hline Pulse & Length (ms) & Dig. Out & A1 & A2 & A3 & A4 & Action \\
\hline 1 & 98 & aceghj & 0.05 & 0.25 & -5 & -5 & MOT \\
2 & 15 & aceghl & 0.5 & 0.25 & -5 & -5 & CMOT \\
3 & 1 & aeghl & 0.6 & 0 & -5 & -5 & optical molasses \\
4 & 0.2 & efghj & 0 & 10 & -5 & -5 & prepare for B field catch \\
5 & 1 & efghj & 0.5 & 10 & -5 & -5 & optical pumping, prepare B field \\
\hline
\end{tabular}

Table F.8: Pulse sequence for subroutine loadcmot2. 


\section{Bibliography}

[1] see notes on operation of vacuum pumps, available at http://www.lesker.com/ newweb/Vacuum_Pumps.

[2] Images from California Institute of Technology lab manual, available at http: //www . its. caltech. edu/ $\sim \mathrm{ph} 76 \mathrm{a} /$.

[3] D. J. Gauthier, private communication.

[4] See catalog available at http://www.parker.com/compumotor/cat/english/BE_ Series.pdf.

[5] D. Steck rubidium line data http://george.ph.utexas.edu/ steck/alkalidata/ rubidium87numbers.pdf.

[6] M H Anderson, J R Ensher, M R Matthews, C E Wieman, and E A Cornell, Observation of Bose-Einstein condensation in a dilute atomic vapor, Science 269 (1995), 198.

[7] M R Andrews, C G Townsend, H J Miesner, D S Durfee, D M Kurn, and W Ketterle, Observation of interference between two Bose condensates, Science 275 (1997), 637.

[8] E Arimondo, M Inguscio, and P Violino, Experimental determinations of the hyperfine structure in the alkali atoms, Rev Mod Phys 49 (1977), 31.

[9] A S Arnold and E Riis, Bose-Einstein condensates in 'giant' toroidal magnetic traps, J. Mod. Optics 49 (1999), 5861.

[10] P R Berman (ed.), Cavity quantum electrodynamics, Academic Press, San Diego, 1994.

[11] N Beverini, E Maccioni, P Marsili, A Ruffini, and F Sorrentino, Frequency stabilization of a diode laser on the Cs D2 resonance line by the zeeman effect in a vapor cell, J. Phys. B 73 (2001), 133.

[12] H M J M Boesten, C C Tsai, J R Gardner, D J Heinzen, and B J Verhaar, Observation of a shape resonance in the collision of two cold Rb-87 atoms, Phys. Rev. A 55 (1997), 636. 
[13] K Bongs, S Burger, S Dettmer, D Hellweg, J Arlt, W Ertmer, and K Sengstock, Waveguide for Bose-Einstein condensates, Phys. Rev. A 63 (2001), 031602.

[14] K Bongs and K Sengstock, Physics with coherent matter waves, Rep. Prog. Phys. 67 (2004), 907.

[15] S N Bose, Plancks gesetz und lichtquantenhypothese, Zeitschrift für Physik 26 (1924), 178.

[16] C C Bradley, C A Sackett, J J Tollett, and R G Hulet, Evidence of Bose-Einstein condensation in an atomic gas with attractive interactions, Phys. Rev. Lett. 75 (1995), 1687.

[17] B Cheron, H Gilles, J Hamel, O Moreau, and H Sorel, Laser frequency stabilization using zeeman effect, J. Physique III 4 (1994), 401.

[18] M A Clifford, G P T Lancaster, R S Conroy, and K Dholakia, Stabilization of an 852nm extended cavity diode laser using the Zeeman effect, J. Mod. Opt. 47 (2000), 1933.

[19] K L Corwin, Z-T Lu, C F Hand, R J Epstein, and C E Wieman, Frequency-stabilized diode laser with the Zeeman shift in an atomic vapor, Appl. Optics 37 (1998), 3295.

[20] F Dalfovo, S Giorgini, L Pitaevskii, and S Stringari, Theory of Bose-Einstein condensation in trapped gases, Rev. Mod. Phys. 71 (1999), 463.

[21] K B Davis, M O Mewes, M R Andrews, N J van Druten, D S Durfee, D M Kurn, and W Ketterle, Bose-Einstein condensation in a gas of sodium atoms, Phys. Rev. Lett. 75 (1995), 3969.

[22] B Deissler, A magnetic trap for evaporative cooling of Rb atoms, Ph.D. thesis, University of Virginia, 2003.

[23] W Demtröder, Laser spectroscopy : Basic concepts and instrumentation, Springer, Berlin, 1996.

[24] A Einstein, Quantentheorie des einatomigen idealen gases, Sitzungsber. der Preuss. Akad. der Wissen., Physik.-Mathem. Klasse (1925), 3.

[25] D G Fried, T C Killian, L Willmann, D Landhuis, S C Moss, D Kleppner, and T J Greytak, Bose-Einstein condensation of atomic hydrogen, Phys. Rev. Lett. 81 (1998), 3811.

[26] S Gupta, K Dieckmann, Z Hadzibabic, and D E Pritchard, Contrast interferometry using Bose-Einstein condensates to measure $h / m$ and $\alpha$, Phys. Rev. Lett. 89 (2002), 140401.

[27] S Gupta, K W Murch, K L Moore nd T P Purdy, and D M Stamper-Kurn, BoseEinstein condensation in a circular waveguide, Phys. Rev. Lett. 95 (2005), 143201. 
[28] T L Gustavson, A Landragin, and M A Kasevich, Rotation sensing with a dual atominterferometer Sagnac gyroscope, Class. Quantum Grav. 17 (2000), 2385.

[29] E W Hagley, L Deng, M Kozuma, M Trippenbach, Y B Band, M Edwards, M Doery, P S Julienne, K Helmerson, S L Rolston, and W D Phillips, Measurement of the coherence of a Bose-Einstein condensate, Phys. Rev. Lett. 83 (1999), 3112.

[30] E Hecht, Optics, 3rd ed., Addison-Wesley, Reading, MA, 1998.

[31] H F Hess, Evaporative cooling of magnetically trapped and compressed spin-polarized hydrogen, Phys. Rev. B 34 (1986), 3476.

[32] J Javanainen and M Wilkens, Phase and phase diffusion of a split Bose-Einstein condensate, Phys. Rev. Lett. 78 (1997), 4675.

[33] W Ketterle, D Durfee, and D Stamper-Kurn, Making, probing, and understanding Bose-Einstein condensates, Proceedings of the Internation School of Physics - Enrico Fermi (1999), 67.

[34] A E Leanhardt, T A Pasquini, M Saba, A Schirotzek, Y Shin, D Kielpinski, D E Pritchard, and W Ketterle, Cooling Bose-Einstein condensates below 500 picokelvin, Science 301 (2003), 1513.

[35] F G Lether, Constrained near-minimax rational approximations to Dawson's integral, Appl. Math. and Comp. 88 (1997), 267.

[36] H J Lewandowski, D M Harber, D L Whitaker, and E A Cornell, Simplified system for creating a Bose-Einstein condensate, J. Low Temp. Phys. 132 (2003), 309.

[37] K B MacAdam, A Steinbach, and C Wieman, A narrow-band tunable diode-laser system with grating feedback, and a saturated absorption spectrometer for $C s$ and $R b$, Am. J. Phys. 60 (1992), 1098.

[38] W M Macek and D I M Davis, Rotation rate sensing with traveling-wave ring lasers, Appl. Phys. Lett. 2 (1963), 67.

[39] E. Majorana, Atomi orientati in campo magnetico variabile, Nuovo Cimento 9 (1932), 43.

[40] P J Martin, B G Oldaker, A H Miklich, and D E Pritchard, Bragg scattering of atoms from a standing light wave, Phys. Rev. Lett. 60 (1988), 515.

[41] N Masuhara, J M Doyle, J C Sandberg, D Kleppner, T J Greytak, H F Hess, and G P Kochanski, Evaporative cooling of spin-polarized atomic hydrogen, Phys. Rev. Lett. 61 (1988), 935.

[42] H J Metcalf and P van der Straten, Laser cooling and trapping, Springer-Verlag, New York, 1999. 
[43] A L Migdall, J V Prodan, W D Phillips, T H Bergeman, and H J Metcalf, First observation of magnetically trapped neutral atoms, Phys. Rev. Lett. 54 (1985), 2596.

[44] C J Myatt, Bose-Einstein condensation experiments in a dilute vapor of rubidium, Ph.D. thesis, University of Colorado, 1997.

[45] S Nakayama, Polarization spectroscopy of $R b$ and $C s$, Optics Communications 50 (1984), 19.

[46] _ Velocity selective optical pumping spectroscopy of D1 lines in alkali atoms, Journal of the Physical Society of Japan 53 (1984), 3351.

[47] __ Optical pumping effects in high resolution laser spectroscopy, Physica Scripta T70 (1997), 64.

[48] Maxim Olshanii and Vanja Dunjko, Interferometry in dense nonlinear media and interaction-induced loss of contrast in microfabricated atom interferometers, condmat/0505358.

[49] K R Overstreet, J Franklin, and J P Shaffer, Zeeman effect spectroscopically locked Cs diode laser system for atomic physics, Rev. Sci. Inst. 75 (2004), 4749.

[50] SE Park, HS Lee, TY Kwon, and H Cho, Dispersion-like signals in velocity-selective saturated-absorption spectroscopy, Opt. Commun. 192 (2001), 49.

[51] T Petelski, M Fattori, G Lamporesi, J Stuhler, and GM Tino, Doppler-free spectroscopy using magnetically induced dichroism of atomic vapor: a new scheme for laser frequency locking, Euro. Phys. J. D 22 (2003), 279.

[52] A Peters, K Y Chung, and S Chu, Measurement of gravitational acceleration by dropping atoms, Nature 400 (1999), 849.

[53] C J Pethick and H Smith, Bose-Einstein condensation in dilute gases, Cambridge University Press, Cambridge, 2002.

[54] W Petrich, M H Anderson, J R Ensher, and E A Cornell, Stable, tightly confining magnetic trap for evaporative cooling of neutral atoms, Phys. Rev. Lett. 74 (1995), 3353.

[55] E Raab, M Prentiss, A Cable, S Chu, and D Pritchard, Trapping of neutral-sodium atoms with radiation pressure, Phys. Rev. Lett. 59 (1987), 2631.

[56] NP Robins, BJJ Slagmolen, DA Shaddock, JD Close, and MB Gray, Interferometric, modulation-free laser stabilization, Opt. Lett. 27 (2002), 1905.

[57] M Saba, T A Pasquini, C Sanner, Y Shin, W Ketterle, and D E Pritchard, Continuous measurement of the relative phase of two Bose-Einstein condensates using light scattering, Science 307 (2005), 1945. 
[58] G Sagnac, L'éther lumineux démontré par l'éffet du vent relatif d'éther dans un interférometre en Allan variance analysis rotation uniforme, C. R. Acad. Sci. 95 (1913), 708.

[59] J Schmiedmayer, M S Chapman, C R Ekstrom, T D Hammond, S Wehinger, and D E Pritchard, Index of refraction of various gases for sodium matter waves, Phys. Rev. Lett. 74 (1995), 1043.

[60] Y Shin, M Saba, T A Pasquini, W Ketterle, D E Pritchard, and A E Leanhardt, Atom interferometry with Bose-Einstein condensates in a double-well potential, Phys. Rev. Lett. 92 (2004), 050405.

[61] Y Shin, C Sanner, G-B Jo, T A Pasquini, M Saba, D E Pritchard W Ketterle, $\mathrm{M}$ Vengalattore, and M Prentiss, Interference of Bose-Einstein condensates split with an atom chip, Phys. Rev. A 72 (2005), 021604(R).

[62] J Söding, D Guery-Odelin, P Desbiolles, F Chevy, H Inamori, and J Dalibard, Threebody decay of a rubidium Bose-Einstein condensate, Appl. Phys. B 69 (1999), 257.

[63] G E Stedman, Ring-laser tests of fundamental physics and geophysics, Rep. Prog. Phys. 60 (1997), 615.

[64] CI Sukenik, HC Busch, and M Shiddiq, Modulation-free laser frequency stabilization and detuning, Opt. Commun. 203 (2002), 133.

[65] Y Torii, Y Suzuki, M Kozuma, T Sugiura, T Kuga, L Deng, and E W Hagley, Mach-Zehnder Bragg interferometer for a Bose-Einstein condensate, Phys. Rev. A 61 (2000), 041602(R).

[66] Y J Wang, D Z Anderson, V M Bright, E A Cornell, Q Diot, T Kishimoto, M Prentiss, $\mathrm{R}$ A Saravanan, S R Segal, and S Wu, Atom michelson interferometer on a chip using a Bose-Enstein condensate, Phys. Rev. Lett. 94 (2005), 090405.

[67] G Wasik, W Gawlik, J Zachorowski, and W Zawadzki, Laser frequency stabilization by Doppler-free magnetic dichroism, Appl. Phys. B 75 (2002), 613.

[68] T Weber, J Herbig, M Mark, H-C Nägerl, and R Grimm, Bose-Einstein condensation of cesium, Science 299 (2003), 232.

[69] D S Weiss, B C Young, and S Chu, Precision measurement of $h / m_{C s}$ based on photon recoil using laser-cooled atoms and atomic interferometry, Appl. Phys. B 59 (1994), 217.

[70] W H Wing, On neutral particle trapping in quasistatic electromagnetic fields, Prog Quant Elect 8 (1984), 181.

[71] S Wu, E J Su, and M Prentiss, Time domain deBroglie wave interferometry along a magnetic guide, Euro. Phys. J. D 35 (2005), 111. 
[72] S Wu, Y J Wang, Q Diot, and M Prentiss, Splitting matter waves using an optimized standing-wave light-pulse sequence, Phys. Rev. A 71 (2005), 043602.

[73] R Wynands and S Weyers, Atomic fountain clocks, Metrologia 42 (2005), S64.

[74] VV Yashchuk, D Budker, and JR Davis, Laser frequency stabilization using linear magneto-optics, Rev. Sci. Instr. 71 (2000), 341.

[75] J Ye, S Swartz, P Jungner, and J L Hall, Hyperfine structure and absolute frequency of the ${ }^{87} R b 5 P_{3 / 2}$ state, Optics Letters 21 (1996), 1280. 